\title{
CDMS DETECTOR FABRICATION IMPROVEMENTS AND LOW ENERGY NUCLEAR RECOIL MEASUREMENTS IN GERMANIUM
}

\author{
A Dissertation \\ by \\ ANDREW KARL JASTRAM \\ Submitted to the Office of Graduate and Professional Studies of \\ Texas A\&M University \\ in partial fulfillment of the requirements for the degree of \\ DOCTOR OF PHILOSOPHY
}

$\begin{array}{ll}\text { Chair of Committee, } & \text { Rupak Mahapatra } \\ \text { Committee Members, } & \text { Rusty Harris } \\ & \text { Bhaskar Dutta } \\ & \text { Rainer Fries } \\ \text { Head of Department, } & \text { George Welch }\end{array}$

December 2015

Major Subject: Physics

Copyright 2015 Andrew Karl Jastram 


\begin{abstract}
As the CDMS (Cryogenic Dark Matter Search) experiment is scaled up to tackle new dark matter parameter spaces (lower masses and cross-sections), detector production efficiency and repeatability becomes ever more important. A dedicated facility has been commissioned for SuperCDMS detector fabrication at Texas A\&M University (TAMU). The fabrication process has been carefully tuned using this facility and its equipment. Production of successfully tested detectors has been demonstrated. Significant improvements in detector performance have been made using new fabrication methods, equipment, and tuning of process parameters. This work has demonstrated the capability for production of next generation CDMS SNOLAB detectors.

Additionally, as the dark matter parameter space is probed further, careful calibrations of detector response to nuclear recoil interactions must be performed in order to extract useful information (in relation to dark matter particle characterizations) from experimental results. A neutron beam of tunable energy is used in conjunction with a commercial radiation detector to characterize ionization energy losses in germanium during nuclear recoil events. Data indicates agreement with values predicted by the Lindhard equation, providing a best-fit k-value of 0.146.
\end{abstract}




\section{ACKNOWLEDGEMENTS}

I have received a great deal of support from others in the process of this work, without which, success would not have been possible.

I would like to thank my friends and family for their constant reminders of the real priorities in my life. They have helped me to stay focused as well as allowed me the much needed breaks in focus to maintain my sanity. In particular, I'd like to thank my wife Kristi for helping me to keep my cool when day to day stresses inevitably arose. I'd like to thank my parents and brother for nurturing my creative endeavors from a young age, and giving me an audience in the same vein.

I'd like to thank my advisor, Dr. Rupak Mahapatra for providing me goals and understanding the work involved in reaching them. I'd also like to thank Dr. Rusty Harris for providing guidance in mastering a process initially foreign to me.

I'd like to thank those with whom I've worked in the labs: Mark Platt, James Phillips, Kunj Prasad, Joel Sander, Sriteja Upadhyayula and JaeWoo Suh.

I'd like to thank those whom provided invaluable help repairing and maintaining the proton accelerator used in this work: Clement Sofka (my pseudo-mentor and trainer), William Baker (my successor), and Tony Restivo (my sounding board for crazy repair ideas).

I'd like to thank Andy Kubik for the crucial last-minute analysis help as well as performing simulation and fitting tasks.

Finally, I'd like to thank Dr. Craig Marianno for lending critical equipment for my work and the CDMS collaboration for providing many exciting opportunities. 


\section{NOMENCLATURE}

TAMU Texas A\&M University

CDMS Cryogenic Dark Matter Search

WIMP Weakly Interacting Massive Particle

(i)ZIP (Interleaved) Z-Sensitive Ionization and Phonon Detector

TES Transition Edge Sensor

aSi Amorphous Silicon

$\mathrm{T}_{c} \quad$ Superconducting Transition Temperature

SUL Soudan Underground Labs

keVnr $\quad$ keV Deposited via Nuclear Recoil

MCA Multi Channel Analyzer

SCA Single Channel Analyzer

LLD Lower Level Discriminator

ULD Upper Level Discriminator

SD Scatter Detector

ICR Incoming Count Rate

DSP Digital Signal Processor

FWHM Full-Width at Half-Maximum

TOF Time of Flight 


\section{TABLE OF CONTENTS}

Page

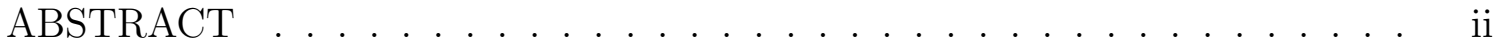

ACKNOWLEDGEMENTS ..................... . . iii

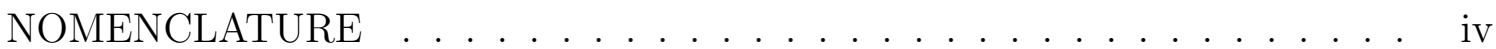

TABLE OF CONTENTS . . . . . . . . . . . . . . . . v v

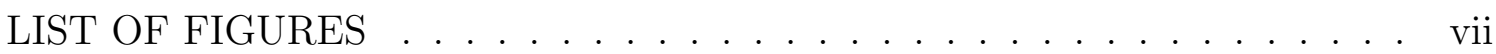

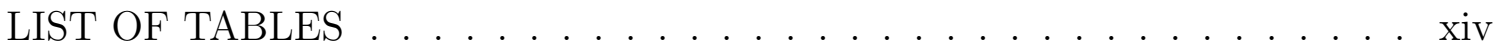

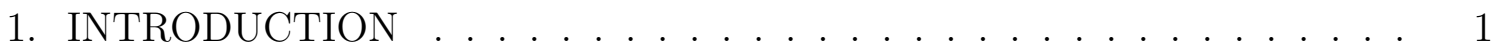

1.1 Observational Evidence of Dark Matter . . . . . . . . . . . . . . . . . 1

1.2 The WIMP Candidate . . . . . . . . . . . . . . . . . . 3

2. CRYOGENIC DARK MATTER SEARCH . . . . . . . . . . . . . 6

2.1 Introduction . . . . . . . . . . . . . . . . . 6

2.2 Location and Shielding . . . . . . . . . . . . . . . . . . . . . . . . . . . . . . . .

2.3 Detectors . . . . . . . . . . . . . . . . 8

2.3.1 Detector Fundamentals . . . . . . . . . . . . . 8

2.4 Detector Limitations . . . . . . . . . . . . . . . . . . . . . . . 10

2.4.1 WIMP Interaction Rate vs. Background Rate . . . . . . . . . 10

2.4.2 Nuclear Recoil Sensitivity . . . . . . . . . . . . . . 11

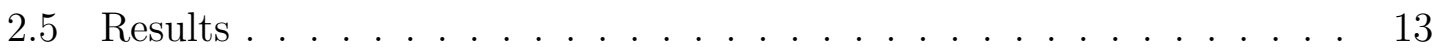

2.6 Future ........................... 14

3. DETECTOR FABRICATION PROCESS AND IMPROVEMENTS . . . . 16

3.1 Fabrication . . . . . . . . . . . . . . . . 16

3.1.1 Substrate Materials . . . . . . . . . . . . . . . . . . . . 16

3.1.2 Alignment and Shaping. . . . . . . . . . . . . . . . 18

3.1.3 Heavy Etch . . . . . . . . . . . . . . . . . . . . . . . . . 19

3.1.4 Lapping and Polishing . . . . . . . . . . . . . . . 20

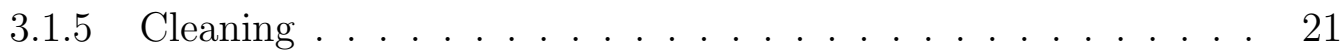


3.1.6 Thin Film Deposition and Tuning . . . . . . . . . . . . . . 23

3.1 .7 Photolithography . . . . . . . . . . . . . . . . . 28

3.1 .8 Inspection, Surgery, and Mounting . . . . . . . . . . . . 33

3.2 Process Tuning, Results, and Improvements . . . . . . . . . . . . 34

3.2 .1 Aluminum Film . . . . . . . . . . . . . . . . . . . 34

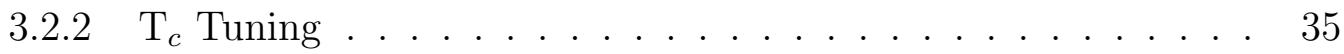

3.2 .3 Conformal Film Deposition _. . . . . . . . . . . 37

3.2.4 a-Si Etch and "Trenching" of iZIP Detectors . . . . . . . . . 40

3.2 .5 Photoresist Studies . . . . . . . . . . . . . . . . 42

3.3 Results to Date . . . . . . . . . . . . . . . . . . . 44

4. LOW ENERGY NUCLEAR RECOIL MEASUREMENTS IN GERMANIUM 47

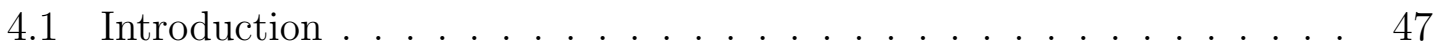

4.2 Experimental Setup . . . . . . . . . . . . . . . . 47

4.2.1 Proton Beam and Neutron Production . . . . . . . . 50

4.2 .2 Detector Setup . . . . . . . . . . . . . . . . 56

4.2.3 Signal vs. Background Event Discrimination . . . . . . . . . 57

4.2.4 Logic and Timing Setup . . . . . . . . . . . . . . . 61

4.2.5 Timing Deviations . . . . . . . . . . . 6 66

4.2.6 Scatter Parameter Choices and Future Considerations . . . . . 68

4.3 Uncertainties . . . . . . . . . . . . . . . . . . . . . . 73

4.3.1 Uncertainty in Neutron Energy _. . . . . . . . . . . 74

4.3.2 Scatter Angle Uncertainties . . . . . . . . . . . . . . . . . 77

4.3.3 Germanium Detector Resolution . . . . . . . . . . . . . . 78

4.3.4 Uncertainties Condensed . . . . . . . . . . . . . . . . . 78

4.4 Analysis and Results . . . . . . . . . . . . . . . . . . . 79

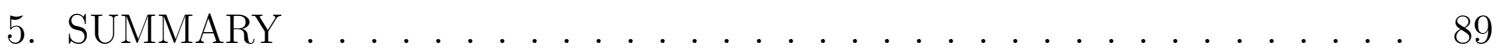

5.1 Detector Fabrication $\ldots \ldots \ldots \ldots$

5.2 Low Energy Nuclear Recoil Measurements . . . . . . . . . . . . . 89

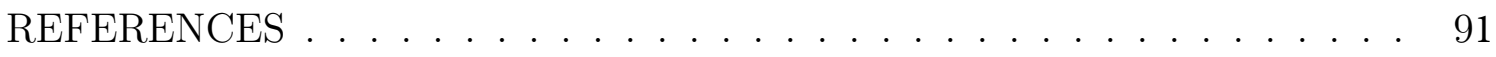

APPENDIX A. DUOPLASMATRON ION SOURCE . . . . . . . . . . . 96

A.1 Overview. . . . . . . . . . . . . . . . . . 96

A.2 Anode . . . . . . . . . . . . . . . 96

A.3 Lens Elements . . . . . . . . . . . . . . . . . . . . . . 99

APPENDIX B. PELLETRON TANDEM ACCELERATOR . . . . . . . . . . 101 


\section{LIST OF FIGURES}

FIGURE

Page

1.1 Composition of the universe showing recent adjustments due to recent measurements by the PLANCK mission [2] . . . . . . . . . . 2

1.2 Images of the "bullet cluster" [4]. In both images, the green lines indicate $\kappa$ contours resulting from weak lensing measurements (an indication of the presence of mass). Left) Color image from Magellan observations showing the region in which lensing observations were made. Right) X-ray images from Chandra showing the interaction of the x-ray emitting component of the cluster. . . . . . . . . . . .

2.1 Comparison of various underground labs based on their effective overburden $[6] \ldots \ldots \ldots \ldots$

2.2 Diagram of shielding used in SCDMS Soudan. . . . . . . . . . . 8

2.3 Cartoon depiction of the evolution of an event within a CDMS detector. Plot on bottom right shows disparate phonon signal amplitudes in each of the four phonon sensors, an effect which can be used to determine the position of the event $\ldots \ldots \ldots \ldots$

2.4 Left) Plot showing the discrepancy in ionization:phonon energy ratio for nuclear recoils vs. electronic recoils in simulated data. Right) Plot of 'yield' (ionization energy divided by phonon energy) vs. phonon energy events from calibration sources, demonstrating the ability to discriminate against electronic recoils. . . . . . . . . . . . .

2.5 Limit plot showing results of previous experiments and project limits of future experiments. . . . . . . . . . . . . . .

2.6 Evolution of CDMS experiments' detector size, payload, and sensor layout. . . . . . . . . . . . . . . . . . 15

3.1 Process flow chart from raw substrate to completion (see Section 3.1). 
3.2 Example RGA spectra of partial pressures in the SEGI process chamber at various atomic masses before (top) and after (bottom) aluminum getter deposition (see Section 3.1.6), demonstrating the efficacy of this process in reducing oxygen and water vapor levels. . . . . . . . .

3.3 Image of a single phonon sensor and magnified inset of TES line and "waterfall" region (arrow indicates waterfall boundary, see Section 3.2.3 for description). Each color corresponds to the exposed film on the final structure: Blue=Aluminum (mask \#1, trilayer mask), Pink=Tungsten (mask \#2, TES mask), Green=a-Si (mask \#3, "trench" mask) (see Section 3.1.7). The central vertical line is the $\sim 2 \mu$ m wide TES, and the large aluminum "fins" are the phonon absorbing structures (see Section 3.2.1). Note: a-Si underlies all metal features. . . . 26

3.4 Detector patterning process (not to scale). Individual film layers are shown throughout the deposition and photolithography process (see Section 3.1.7). Gray=Substrate, Green=a-Si, Blue=Aluminum, Pink=Tungsten, and Red-brown=Photoresist. . . . . . . . . . . .

3.5 Diagram depicting the tungsten overhang issue caused by the isotropic aluminum etch process (not to scale). Figure from [13]. . . . . . . . . 30

3.6 Left) Example of an un-etched section of Aluminum, causing a possible short. Right) Example of photoresist failure allowing unwanted aluminum etching, breaking circuit continuity, and requiring a "surgery" wire bond. Both examples were likely caused by particulates on the detector surface during early processing. . . . . . . . . . . . .

3.7 Left) XRD spectra showing discrimination between samples of differing $\alpha: \beta$ concentrations and their resulting $\mathrm{T}_{c}$ 's. Also marked are the locations of the peaks of pure $\alpha$ and $\beta$ phase films. An algorithm fitting two Gaussian functions (centered at these values) provides an estimate of phase ratios, and therefore $\mathrm{T}_{c}$ 's, of the films. Right) Plot showing correlation of critical temperature vs. sheet resistance of similar thickness films $(40 \pm 4 \mathrm{~nm})$ [13]. These room temperature characterization methods allow film deposition parameters to be tuned without the time or monetary expense of dilution refrigerators (see Section 3.2.2). 
3.8 Left) Example of $\mathrm{W}_{c}$ variation (in $\mathrm{mK}$ ) across a sample substrate face from the previous CDMS deposition system. Middle) $\mathrm{T}_{c}$ variation of same film after ion implant compensation (see Section 3.2.2). Right) $\mathrm{T}_{c}$ measurements (see Table 3.2) from the 8 phonon channels of G9F, a detector fabricated at TAMU, demonstrating $\mathrm{T}_{c}$ uniformity without

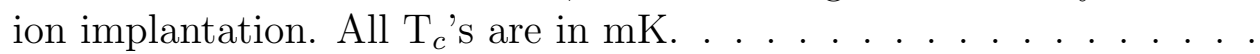

3.9 Close-up and SEM image of "waterfall" boundary (see Section 3.2.3). Location on phonon sensor and perspective are indicated by the arrow, referencing Figure 3.3. . . . . . . . . . . . . . . . . .

3.10 Left) SEM images of overhang discontinuity issue. Middle) Conformal sidewall deposition utilizing overhang etch, resulting in robust film continuity (see Section 3.2.3). Right) SEM image of FIB-prepared cross-section of waterfall feature, showing conformal tungsten deposition. FIB image courtesy of Evans Analytical Group. . . . . . . . .

3.11 Left) Diagram of iZIP detector geometry and design used in SuperCDMS Soudan showing alternating biased charge collecting rails and 0V phonon rails (see Section 3.2.4). Figure from [20]. Right) Simulation of iZIP internal field lines, showing strong tangential electric fields at the surface and a uniform drift field in the detector bulk, a method proven to discriminate against the previously problematic surface events. Figure from [21] . . . . . . . . . .

3.12 SEM image of a photoresist feature's cross-section. Due to improper UV exposure, this feature's sidewalls angle outwards, protecting a wider area of film from etching underneath, resulting in widening of circuit features. Note: This layer's thickness of $1.66 \mu \mathrm{m}$ corresponds to a previous spin coat recipe, using S1813 photoresist (see Section $3.2 .5)$.

3.13 Left) Phonon pulses from detector G9F, fabricated at TAMU. Right) Calibration spectrum from detector G9F, clearly showing the $356 \mathrm{keV}$ $\mathrm{Ba}^{133}$ peak (see Section 4.4). . . . . . . . . . . . . . . . . 45

$3.14100 \mathrm{~mm} \times 33.3 \mathrm{~mm}$ detectors fabricated at TAMU. . . . . . . . . . . . 46

4.1 Current measurements of the Lindhard factor in germanium [32] . . . 48

4.2 Neutron rate (measured with a $\mathrm{BF}_{3}$ counter enclosed in polyethylene case) vs. bending magnetic field (a measure of incident proton energy). The threshold in this case is measured within $21 \mu \mathrm{T} . \ldots . . .$. 
4.3 Neutron energy (solid line) and neutron detection rate (dashed line) vs. bending magnetic field. Neutron rate is measured with a $\mathrm{BF}_{3}$ counter enclosed in polyethylene. . . . . . . . . . . . . . . 50

4.4 Diagram of the proton beam setup, neglecting steering, focusing, and measurement components (see Section 4.2.1). Not to scale. . . . . .

4.5 Plot of neutron energies at various angles for given proton energies (from Equation 4.3). . . . . . . . . . . . . . .

4.6 Left) Example neutron spectra of the approximate energy used in this work. Note, the angular dependence is minimal at $0^{\circ}$ (used in this experiment), and $180^{\circ}$. Right) Demonstration of sources of uncertainty in incident neutron energy. The x-axis spread in energy is due to the solid angle of the detector and the angular dependence of neutron production, resulting in a $0.4 \%$ uncertainty based on the target detector and configuration used in this work. The vertical shift is due to uncertainties in the proton energy at the point of the ${ }^{7} \mathrm{Li}(\mathrm{p}, \mathrm{n})^{7}$ Be reaction (see Section 4.3). . . . . . . . . . . . . . .

4.7 Example calibration spectrum obtained with the Ge detector. This spectrum is the result of an ${ }^{55} \mathrm{Fe}$ source on a sheet of aluminum foil on the detector window. Three peaks are used for calibration: A) Al $\mathrm{K} \alpha($ from the foil $)=1.48 \mathrm{keV}, \mathrm{B}) \mathrm{Mn} \mathrm{K} \alpha\left(\right.$ from the $\left.{ }^{55} \mathrm{Fe}\right)=5.89 \mathrm{keV}$, and C) Pile-up of two simultaneous Mn K $\alpha$ events $=11.78 \mathrm{keV}$. These three peaks provide a good measure of linearity and resolution across the full scale of the MCA. . . . . . . . . . . . . . . . . . .

4.8 Diagram showing the end-beam setup. Inset) Diagram of scatter detector shielding and collimation. . . . . . . . . . . . 57

4.9 Effect of scatter detector collimator, as simulated in GEANT, showing the peak narrowing due to the polyethylene without loss of peak

4.10 Left) Scatter detector data showing which pulse heights correspond to the ROI in ionization energy measured in the Ge detector. This population determines hardware settings for upper- and lower-level discriminators for accepting scatter detector pulses. Right) Histograms showing the ROI in each detector under these discriminator settings. Neutron scatter peak is clearly visible in all three. See Section 4.2.3 . 
4.11 Display of 2 channel scope used to monitor and analyze timing information. The red channel is the 'analog' Ge ionization energy channel (an auxiliary output of the MCA) which has an amplitude proportional to the ionization energy. The blue channel measures the two combined timing pulses: the ICR pulse from the MCA (signaling the detection of a Ge ionization event) and the scatter detectors' combined discriminator output, signaling a scatter detector ionization event. Inset) Zoomed image of a scatter detector pulse followed by an ICR pulse. This gap is used to define the timing settings in the timing and logic hardware (see Section 4.2.3) . . . . . . . . . . .

4.12 Trigger timing data. Left) $\sim 30 \mathrm{keVnr}$ events before fine tuning, showing the ROI population is being excluded at higher $(>\sim 550 \mathrm{nS})$ trigger spacings. Middle) Data from remainder of run after timing adjustments were implemented. ROI is clearly well defined inside of this parameter space. C) 16keVnr events (after further tuning) showing shifted ionization energy measured. . . . . . . . . . . . . . 61

4.13 Block diagram of triggering circuit and DAQ setup. . . . . . . . . .

4.14 Inherent delay of Ge trigger after scatter detector trigger vs. Ge ionization energy. Different colors represent different targeted nuclear recoil energies. Rapid increase of delays with decreasing energy (below $\sim 3 \mathrm{keV}$ ) is unexpected and needs to be accounted for in the timing and logic settings. . . . . . . . . . . . . . . .

4.15 Plots showing timing and energy correlation in Ge detector using a ${ }^{22} \mathrm{Na}$ source. Left) Fast discriminator shaping mode set to 'low energy'. Right) Fast discriminator shaping mode set to 'normal'. While trigger efficiency and detector resolution is degraded, timing becomes more uniform. . . . . . . . . . . . . . . . . .

4.16 Plot showing the timing disparity using higher energy gamma events, including the $511 \mathrm{keV}^{22} \mathrm{Na}$ photo-peak. . . . . . . . . . . . .

4.17 Plot of neutron rate (solid line) and energy (dashed) vs. incident proton energy[33]. Note the sharp turn-on at threshold, which is exploited in the energy calibration process. . . . . . . . . . . . .

4.18 Plot of GEANT simulated events scattered at various angles, demonstrating the advantage of shallow angle scatters. Scatter angle in degrees, from left to right: $22.5,45,67.5,90,112.5,135,157.5$. . . 
4.19 Plot of tagged neutron scatters vs. scatter angle, showing shallow angle preference (simulated in GEANT) . . . . . . . . . . 71

4.20 Plot of simulated germanium energy deposition spectra from various scatter detector distances, demonstrating the effect on rate and energy spread. Distances in $\mathrm{cm}$, from smallest peak to largest: 34.3, 30.5,

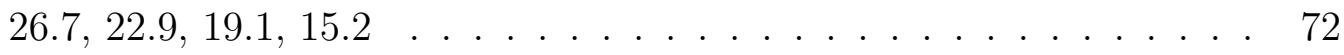

4.21 Bending magnet field over time. 0.00363\% (10 $\mu \mathrm{T})$ maximum deviation in 20 Minutes. . . . . . . . . . . . . . . . 75

4.22 Stopping power of LiF for incident protons. This plot shows all proton energies used in this work (see Section 4.3.1). Stopping power values provided by NIST pstar database [35].

4.23 Full spectrum of measured event energies. This is the spectrum obtained by recording all events (no scatter detector requirement. The neutron shelf can easily be seen, marked by the endpoint at which $180^{\circ}$ scatters have occurred. This is the expected shape of the background due to random coincidence events. . . . . . . . . . . . . .

4.24 Ionization measurements at 10 different recoil energies, using the sigma values of the Gaussian fits for the errors, a conservative approach. . . 82

4.25 Ionization measurements at 10 different recoil energies, using the statistical uncertainty in the mean for the errors. This method does not account for systematic effects. . . . . . . . . . . . . . 83

4.26 Raw data and fit for $4.0 \mathrm{keV}$ nuclear recoil depositions. . . . . . . . 84

4.27 Raw data and fit for $5.0 \mathrm{keV}$ nuclear recoil depositions. . . . . . . . 84

4.28 Raw data and fit for $6.3 \mathrm{keV}$ nuclear recoil depositions. . . . . . . . 85

4.29 Raw data and fit for $8.0 \mathrm{keV}$ nuclear recoil depositions. . . . . . . . 85

4.30 Raw data and fit for $10.1 \mathrm{keV}$ nuclear recoil depositions. . . . . . . 86

4.31 Raw data and fit for $12.7 \mathrm{keV}$ nuclear recoil depositions. . . . . . . 86

4.32 Raw data and fit for $16.0 \mathrm{keV}$ nuclear recoil depositions. . . . . . . 87

4.33 Raw data and fit for $20.1 \mathrm{keV}$ nuclear recoil depositions. . . . . . . 87

4.34 Raw data and fit for $25.3 \mathrm{keV}$ nuclear recoil depositions. . . . . . . 88 
A.1 Diagram of the duoplasmatron ion source used in this work (not to scale). A) Tungsten filament (see Figure A.2, B) Zwischen cylinder/electrode, C) Electromagnet, D) Zwischen-position adjustment screw (1 of 4), E) Anode (see Figure A.3, F) Bypass valve, G) Lens element contact finger ( 1 of 6 , see Figure A.4), $\mathrm{H}$ ) $\mathrm{H}_{2}$ gas input, I) Permanent magnet to deflect electrons out of beam, J) Focus lens element, K) Extractor lens element, L) Field shaping ring (1 of 3), M) 150 Mohm resistor ( 1 of 7 in resister bridge). . . . . . . . .

A.2 Example filament used in this ion source. It is 0.027 " diameter tungsten wire, cut to 2.75 ", then manually bent using needle-nose pliers.

A.3 Left) Comparison of damaged anode next to its replacement. Note the damage near the aperture from extended abuse from the beam staying in a single spot. Right) The rear (beam exiting) side of the damaged anode, showing that the damage from the front, not only punched through, but caused an irregular shape in the aperture. This extra hole resulted in extra errant beam, which subsequently loaded down the lens elements (their power supplies), preventing successful beam operation. . . . . . . . . . . . . . . . . . .

A.4 Ion source lens elements and accelerator column simulation jig. Left) Yellow circles indicate focus electrode "fingers", whereas red circles indicate the equivalent component locations on the extractor electrode (missing "finger" on left has since been replaced. Center) Jig created to simulate the inner ring contact points of the accelerator column, allowing lens element "fingers" to be shaped appropriately before blind assembly. Right) Jig mounted on ion source to test the configuration of contact "fingers". . . . . . . . . . . . . . . . .

B.1 Diagram of pelletron charging system [37]. Chain and pulleys rotate clockwise (see reference for online animation). . . . . . . . . . 101 


\section{LIST OF TABLES}

TABLE

Page

3.1 Bake times for various substrate sizes. Thin (practice) wafers soft bake for $1 \mathrm{~m} 50 \mathrm{~s}$ on a $115^{\circ} \mathrm{C}$ hot plate, and hard bake for 15 minutes

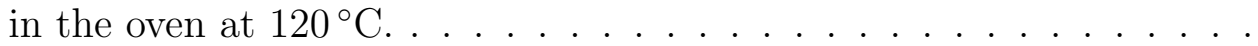

3.2 Critical temperatures and "normal" resistance values $\left(\mathrm{R}_{n}\right)$ for the 8 phonon channels of detector G9F (see Section 3.2.2). $\mathrm{R}_{n}$ is the resistance of the channel while the aluminum is superconducting, but the tungsten is normal (held at a temperature significantly above its $\mathrm{T}_{c}$ ). Note: Channels A1 and A2 are outer channels (see Figure 3.8) and have higher $\mathrm{R}_{n}$ values due to their sensor layout. . . . . . . . . . . . . 39

4.1 Lab frame angles used in this work and their corresponding center of mass frame angles. . . . . . . . . . . . . . . . . . . . . . . 73

4.2 Data from all runs used in this analysis. Variables (left to right): incident neutron energy, scatter angle (lab-frame), energy deposited by nuclear recoil, energy measured, sigma value of Gaussian peak-fit, and $\%$ ionization energy measured. . . . . . . . . . . . . . . 82 


\section{INTRODUCTION}

For centuries, mankind has searched simultaneously at the largest and smallest scales of its surroundings. From telescope to microscope, we attempt to observe the extremes, always pushing the 'limits', constantly proving how temporary they truly are. In this pursuit, the unexplainable always stands out as the most interesting and exciting. Rather than answers documented on paper, in files, etc., questions are raised, quests begun, truths sought out. This situation is no more evident than in the search for dark matter.

Perhaps the most exciting aspect of this search is the evidence that it not only appears to be incredibly abundant (roughly 5 times the abundance of baryonic matter), but the fact that it is expected to exist in our own backyard. These two components immediately raise questions in a scientist's mind: what is it and how can it be detected?

\subsection{Observational Evidence of Dark Matter}

Arguably the biggest breakthrough spawning these questions was that made by Fritz Zwicky in 1933. In his famous paper "The redshift of extragalactic nebulae" [1], he noticed that the disparities in red-shifts of 'nebulae' ${ }^{1}$ (corresponding to their orbital velocities) in the Coma Cluster were too large to be explained by the visible matter present. The measurements also showed that the amount of 'missing' matter was actually larger than the detectable matter. Since then, many galaxies have been observed for this effect, showing the mass:light ratio found by Zwicky was not anomalous, but actually typical.

The scientific community received another 'prodding' in 1974 in the form of a

\footnotetext{
${ }^{1}$ We now know that these were actually galaxies that he was observing.
} 

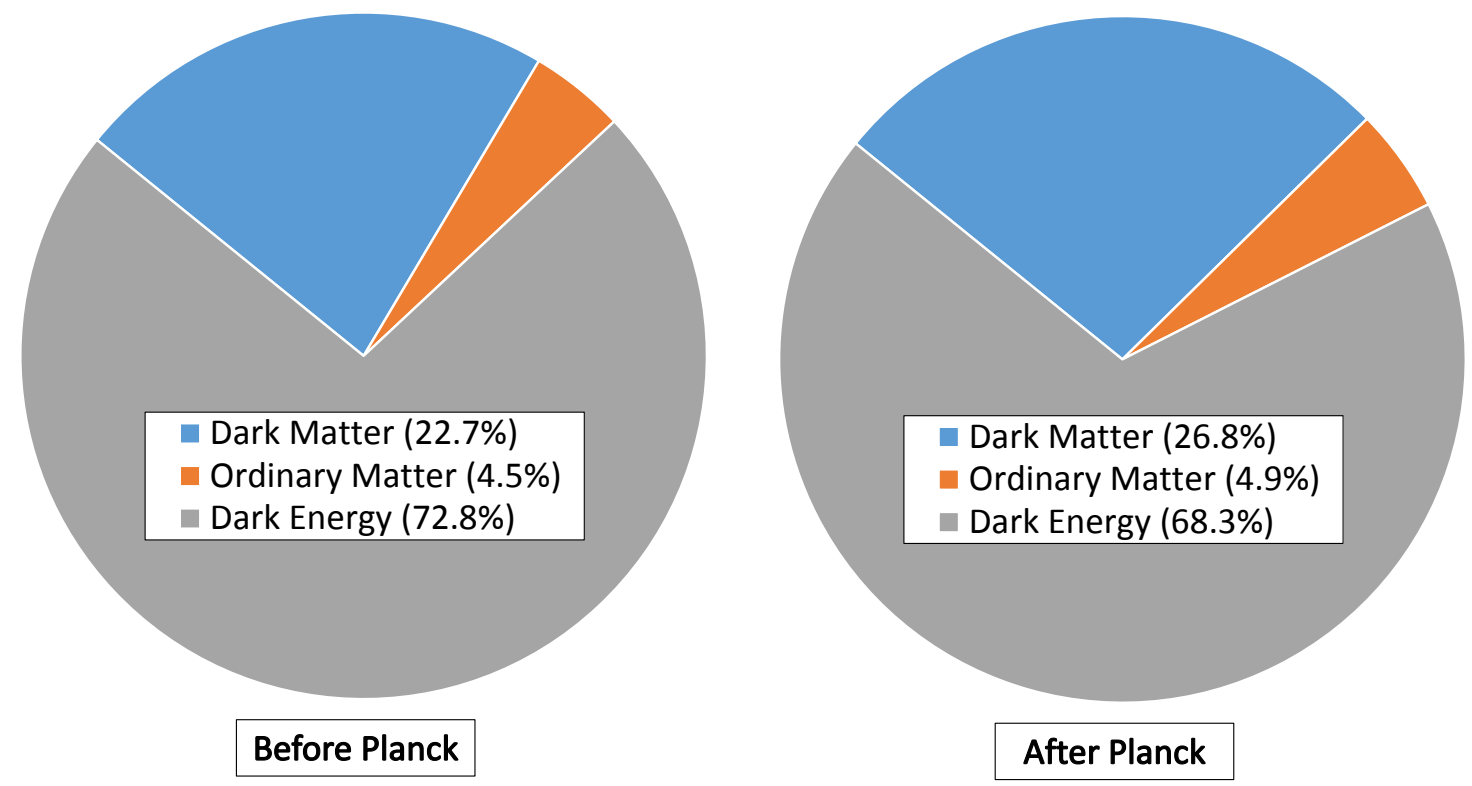

Figure 1.1: Composition of the universe showing recent adjustments due to recent measurements by the PLANCK mission [2].

Nature paper by Einasto et al. [3]. In this paper, a much more robust measurement of the graviational mass:luminous mass ratio was made. Such measurements as this, and those subsequent (arguably pioneered by this work) have been critical in the dark matter field.

While the relative abundance of this dark matter component is still shifting as new measurements and observations are made (see Figure 1.1), these measurements generally agree and emphasize that it is indeed much more abundant than ordinary matter.

Perhaps the most visually compelling evidence for dark matter comes from observations made of the merging cluster 1E 0657-558 (often referred to as the "bullet cluster") by Clowe et. al [4] (see Figure 1.2). In this merging event, two separate galaxy concentrations have passed through each other, providing a chance to observe 

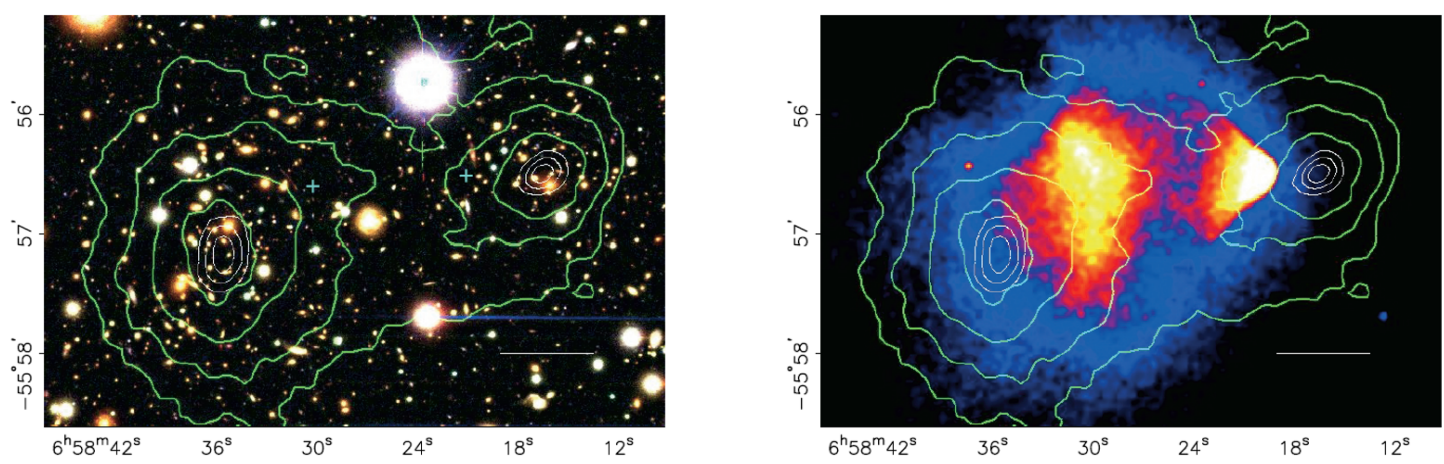

Figure 1.2: Images of the "bullet cluster" [4]. In both images, the green lines indicate $\kappa$ contours resulting from weak lensing measurements (an indication of the presence of mass). Left) Color image from Magellan observations showing the region in which lensing observations were made. Right) X-ray images from Chandra showing the interaction of the x-ray emitting component of the cluster. Reprinted with permission.

their interaction. The dominant visible matter component of each concentration, the X-ray plasma, appears to have been dragged through the other, as expected in this interaction. However, when mapping the location of the mass in the cluster, the majority of it appears to have passed through unimpeded. Without an additional, 'dark' source of matter, the mass concentration would instead tend to closely follow the X-ray plasma.

While there is an abundance of other observational evidence in the argument for dark matter, this is well documented in other sources and will not be covered further here.

\subsection{The WIMP Candidate}

To execute a search for this dark matter component, one must first decide where to look. In a simplest form, one can assume that dark matter consists of one type of particle with a scientifically motivated set of assumed attributes, then devise a way to either detect it or rule out its existence. In this case, the target particle is 
defined as the WIMP (weakly interacting massive particle). Based on cosmological, observational, and theoretical evidence, a particle with a cross-section of somewhere near the weak-interaction scale and of course, mass, fits the profile. The mass, and thus its constituent particles should be distributed throughout our galaxy, meaning the sun, and therefore the Earth, should be continuously flying through it. From a detector's point of view, the dark matter is continuously flying through us with a velocity near that of the sun with a rare chance of colliding with ordinary matter (such as a germanium nucleus in a dark matter detector). The energy deposited in such a detector (with nucleus of mass $M_{T}$ ) by a WIMP with kinetic energy $\mathrm{E}$ and mass $M_{D}$, scattering at an angle $\theta$ is given by the following[5]:

$$
E_{R}=\frac{\operatorname{Er}(1-\cos \theta)}{2}
$$

where

$$
r=\frac{4 M_{D} M_{T}}{\left(M_{D}+M_{T}\right)^{2}}
$$

With an expected dark matter density in our local region and an energy spectrum from candidate dark matter particles, one can determine a range of masses and interaction cross-sections to attribute to a dark matter candidate that would explain the data. The expected recoil spectrum measured from such an experiment should roughly take the exponential form (from [5]) :

$$
\frac{d R}{d E_{R}}=\frac{R_{0}}{E_{0} r} e^{-E_{R} / E_{0} r}
$$

where $\mathrm{r}$ is given in equation $1.2, E_{R}$ is the recoil energy, $E_{0}$ is the most probable incident dark matter particle kinetec energy, $\mathrm{R}$ is the event rate per unit mass, and 
$R_{0}$ is the incident rate.

A Maxwellian dark matter distribution throughout the galaxy is assumed, providing the follwing relation:

$$
f\left(v, v_{E}\right)=e^{-\left(v+v_{E}\right)^{2} / v_{0}^{2}}
$$

where $v$ is the incident velocity, $v_{E}$ is Earth's velocity relative to the dark matter cloud, and $v_{0}$ is the galactic rotation velocity. For a dark matter particle mass $M_{D}=10-1000 \mathrm{GeVc}^{-} 2$ range, typical recoil energies are expected to be in the 1-100 keV range.

However, more commonly, experiments do not see evidence of such a particle. In this case, an exclusion limit is placed on the dark matter parameters, which incrementally push to lower masses and cross-sections (see Section 2.5). it should be noted that these limits rely heavily on measurements such as those made in Section 4 of this work. 


\section{CRYOGENIC DARK MATTER SEARCH}

\subsection{Introduction}

The CDMS (Cryogenic Dark Matter Search) experiment uses semiconductor (silicon and germanium) detectors for the direct detection of WIMPs. These detectors directly measure the energy deposited by particle interaction events in the form of ionization and phonon energy. The sensitivities achieved by these detectors combined with the low background environment in which they are operated allows for one of the most competitive dark matter searches in the field.

\subsection{Location and Shielding}

The Super CDMS experiment (the most recent incarnation) is currently installed in the Soudan Underground Laboratory (SUL) located in Soudan, Minnesota. SUL is located $2340 \mathrm{ft}$ below the surface (with an iron-rich overburden). This results in a 2000 meter water equivalent overburden (see Figure 2.1). Significant overburdens are required in dark matter experiments such as these to reduce the background event rate due to cosmic sources. In the case of SUL, a reduction of $\sim 5$ orders of magnitude is achieved purely from the location. Even with this reduction, the experiment would not be successful without further shielding. This is meant to block the few muons (and their by-products) that still make their way into the lab, as well as radiogenic backgrounds from surrounding materials.

This shielding is implemented in the form of lead and polyethylene layers (see Figure 2.2). This consists of four main layers, from outer- to inner-most:

1. Outer polyethylene layer to block and/or moderate incident neutrons

2. Low activity lead to block gammas 
3. Ancient lead to block gammas and radiation from the less pure outer lead

4. Inner polyethylene to block penetrating neutrons and additional neutrons that may be produced in the lead

This shielding reduces the background rate to that which is acceptable for a WIMP search, leaving the WIMP rate insignificantly affected.

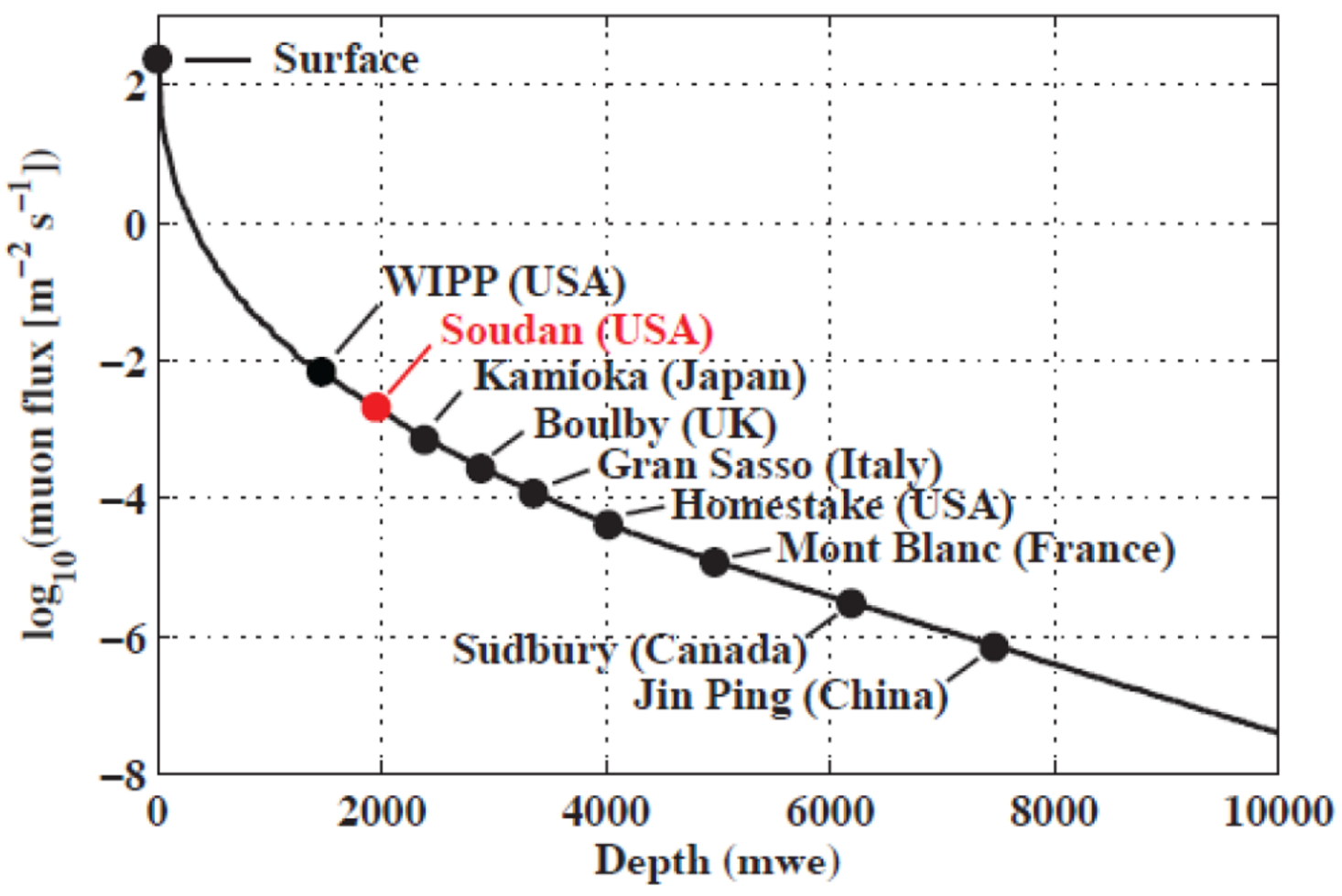

Figure 2.1: Comparison of various underground labs based on their effective overburden, reprinted with permission from [6]. 


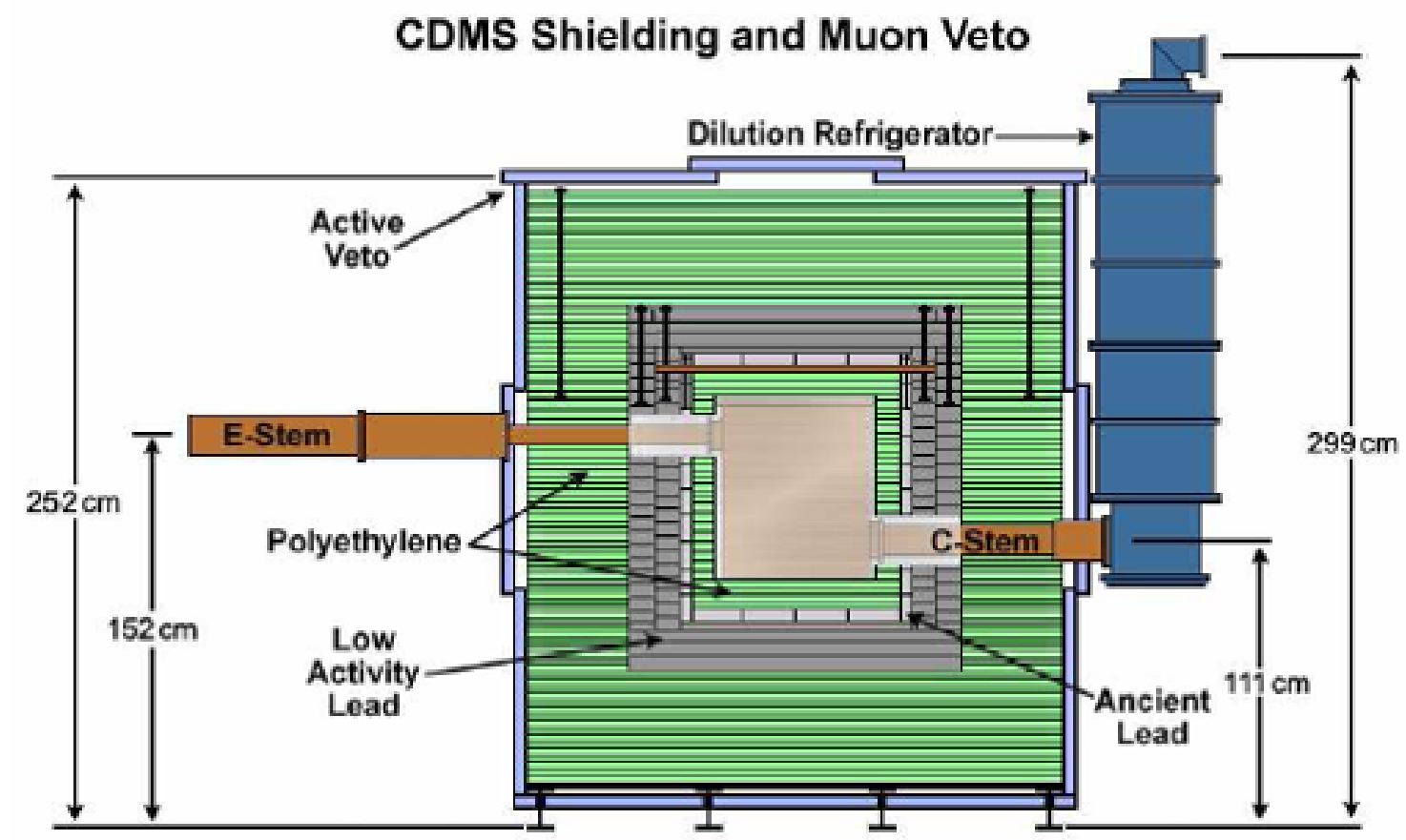

Figure 2.2: Diagram of shielding used in SCDMS Soudan.

\subsection{Detectors}

\subsubsection{Detector Fundamentals}

The detectors used in this experiment are instrumented crystals of germanium and silicon (see Section 3 for information on the fabrication process and sensor design/physics). At the most fundamental level, they monitor energy depositions incident on Si or Ge nuclei in the case of nuclear recoils (in the case of neutrons and WIMPs), or depositions in the electron system (in the case of gammas and betas). The later obviously being the background, needs to be discriminated against to appropriately monitor the former (the signal region). To do this, two types of energy are recorded for each event: phonon and ionization.

The phonon energy is a measurement of the semiconductor system's physical 
recoil to the event, regardless of incident particle type, and is thus termed the 'true energy'. This recoil energy immediately produces optical phonons, which propagate through the crystal, reflecting on the surfaces, until they have down-converted to an energy at which they can be absorbed by the phonon sensors.

The ionization energy is a measurement of the electron-hole pairs produced in the event. These free charge carriers are drifted to their respective electrodes by an applied electric field (see Figure 2.3). As they are drifted, the charge carriers inherently produce phonons as well, and this must be accounted for in the phonon energy measurement. Under certain conditions, these secondary phonons can also be utilized to make more sensitive ionization measurements (see Section 2.4.2).

There is a discrepancy between nuclear and electronic recoils in the amount of ionization energy measured for a given event energy deposition (see Figure 2.4). This discrepancy allows the discrimination of nuclear vs. electronic recoils, essentially eliminating the otherwise dominant background in this experiment. For low energy nuclear recoils, the ionization measured is around $20-30 \%$ of that measured for a similar energy electronic recoil. This ratio is the so-called 'Lindhard Factor', and is not precisely known in the low energy range. The ionization energy scale for these detectors is calibrated using gamma sources, while the Lindhard Factor is determined using a combination of neutron calibration sources $\left({ }^{252} \mathrm{Cf}\right)$, Lindhard model extrapolations, and other experimental data (such as that measured in Section 4 of this work). However, the calibration with ${ }^{252} \mathrm{Cf}$ proves difficult at low energies as the uncertainty of the ionization:phonon energy ratio becomes quite large at low energies. Evidence of this can be seen in Figure 2.4: apparent as the nuclear recoil 'band' broadens drastically as $\mathrm{E}_{\text {recoil }}$ approaches zero. Lindhard model extrapolation has its downsides as well: extrapolation is only as effective as the model itself. While no strong evidence for deviations from the Lindhard model in germanium exists, further 
measurements need to be made to make confident claims based on it and to nail down parameters within which at present, are not precisely known. Other measurements of this 'Lindhard Factor' are useful to the extent of the energies at which they are made, and the error in said measurements. This is the driving principle behind the measurements made in Section 4 of this work.

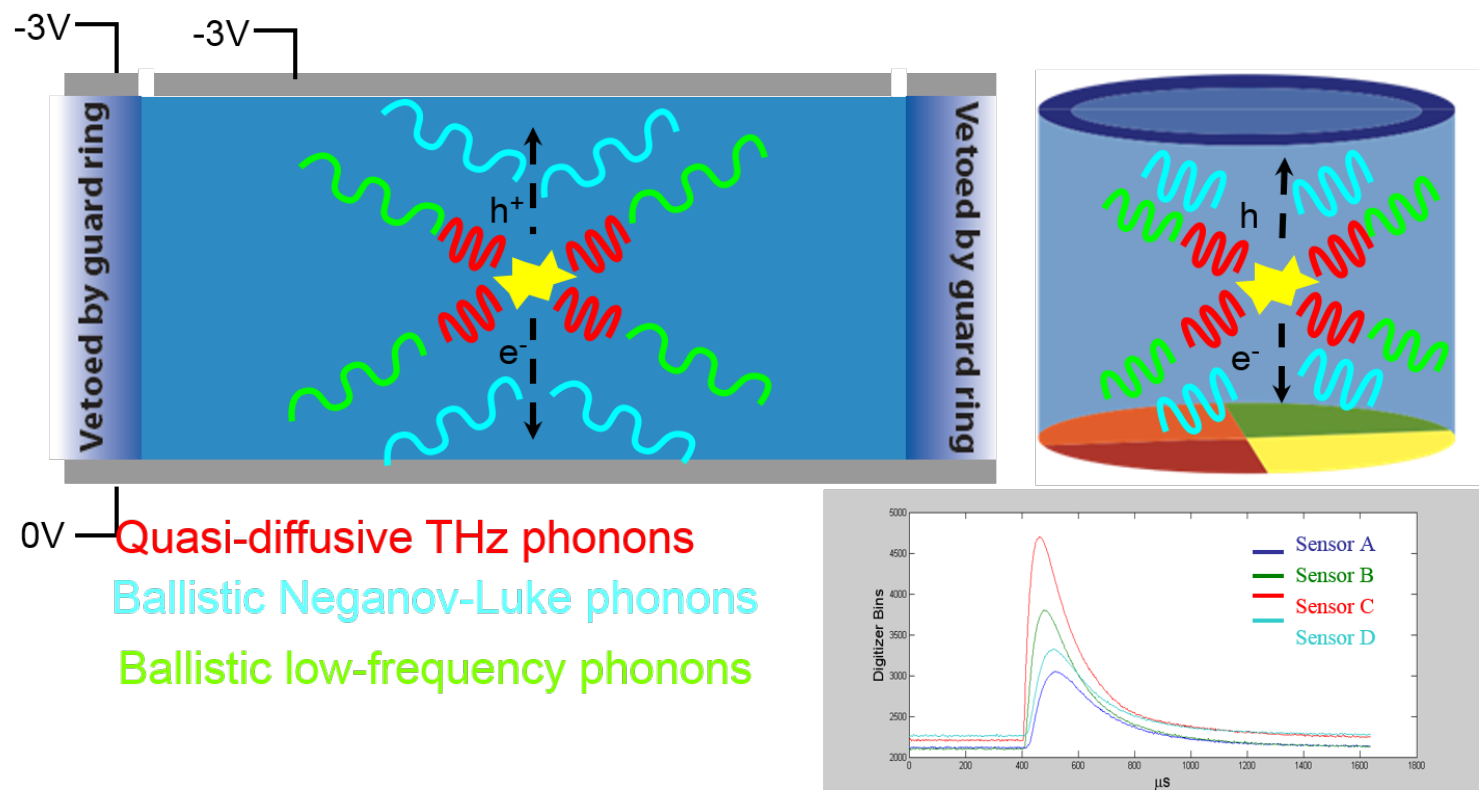

Figure 2.3: Cartoon depiction of the evolution of an event within a CDMS detector. Plot on bottom right shows disparate phonon signal amplitudes in each of the four phonon sensors, an effect which can be used to determine the position of the event.

\subsection{Detector Limitations}

\subsubsection{WIMP Interaction Rate vs. Background Rate}

Due to the very low cross-section expected of the WIMP, large detector masses must be used in its detection. The detection rate scales with the detector mass, so gains can be made very quickly with scalable technologies. Due to the modular 

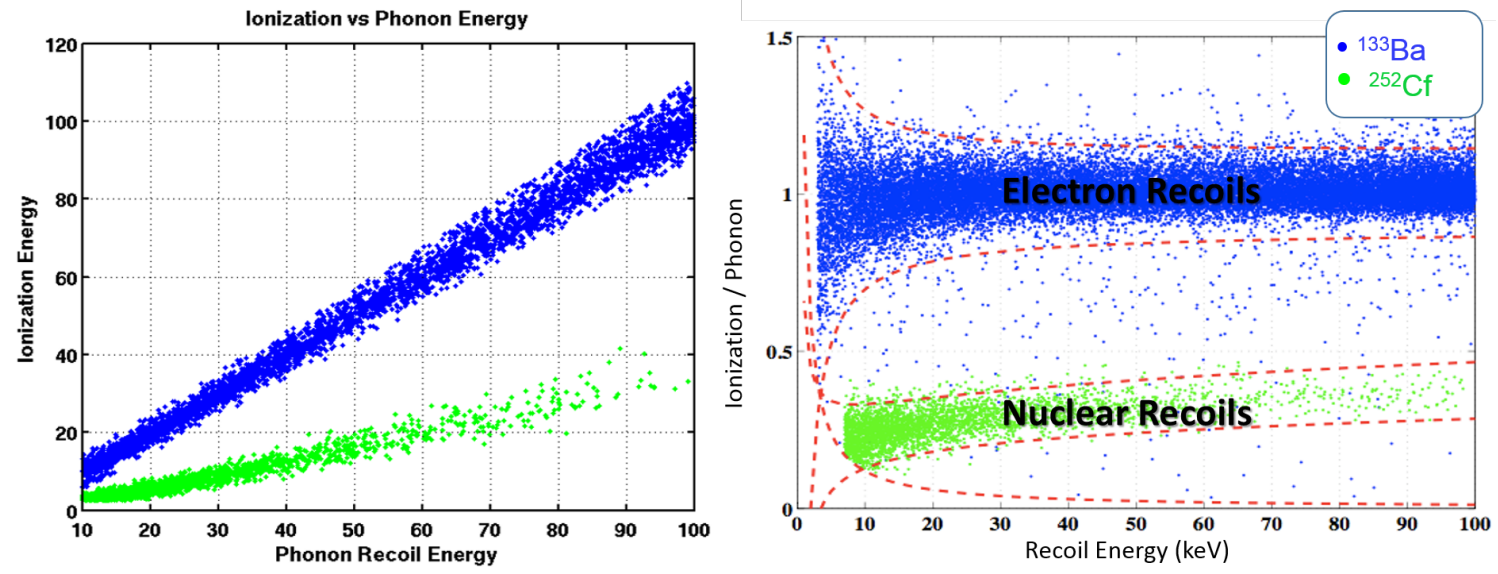

Figure 2.4: Left) Plot showing the discrepancy in ionization:phonon energy ratio for nuclear recoils vs. electronic recoils in simulated data. Right) Plot of 'yield' (ionization energy divided by phonon energy) vs. phonon energy events from calibration sources, demonstrating the ability to discriminate against electronic recoils.

design of the CDMS experiment, this is a simple, yet costly, issue of scaling up the number of detectors. However, this is not an optimal solution, as it inherently scales up corresponding electronics and other detector specific components $(\$ \$)$ as well as scaling up the detector surface area. The rate of many of the dominant background event types scales with surface area (but not volume), so a better solution (scientifically and economically) is to scale up the size of each detector. The detector fabrication process is a complex and delicate one, so scaling up in size is no trivial task. Advances in handling and processing during the fabrication process (described in Section 3) have allowed the successful scaling up of detector mass while maintaining (or even improving) throughput to make a large detector payload production possible in both time and cost.

\subsubsection{Nuclear Recoil Sensitivity}

Due to reduced ionization energy in nuclear recoil events, a successful detector must not only be incredibly sensitive to low ionization energies, it must be well 
calibrated at those energies.

As mentioned in Section 2.3.1, the drifting of charge carriers through the detector produces additional phonons along the way. The quantity of these phonons, termed 'Neganov-Luke' phonons, scales with the electric field through which the carriers are drifted and is proportional to the quantity of liberated charge carriers (i.e. the ionization energy). In this way, an external electric field can be used to amplify the ionization signal in the form of phonons without increasing the noise in the system, a technique called voltage assisted calorimetric ionization detection. This equates to a handle at which signal:noise can be tuned at will (until detector limitations are reached by the applied voltage). This effect has been utilized by this group to produce detectors with a baseline noise of $\sim 7 e V[7]$, with the theoretical capability of further improvement by incorporating minimal design changes.

While these incredibly sensitive detectors allow the detection of much smaller depositions of energy, they are only as good as their calibration (when used in WIMP searches). This is due to the factors mentioned in the beginning of this section. While the measurements carried out in this work (Section 4) are very important for current and future WIMP searches using germanium, they are not sufficient for calibrating the ultra low threshold detectors described above. However, this work has served to set up a facility and procedure in which to carry out these measurements at lower energies allowing these new detectors to be properly calibrated in the future.

This sensitivity and careful calibration opens up not only the ability to probe lower WIMP masses, but also allows for the possibility for detection of coherent neutrino scattering, a process never before measured directly. 


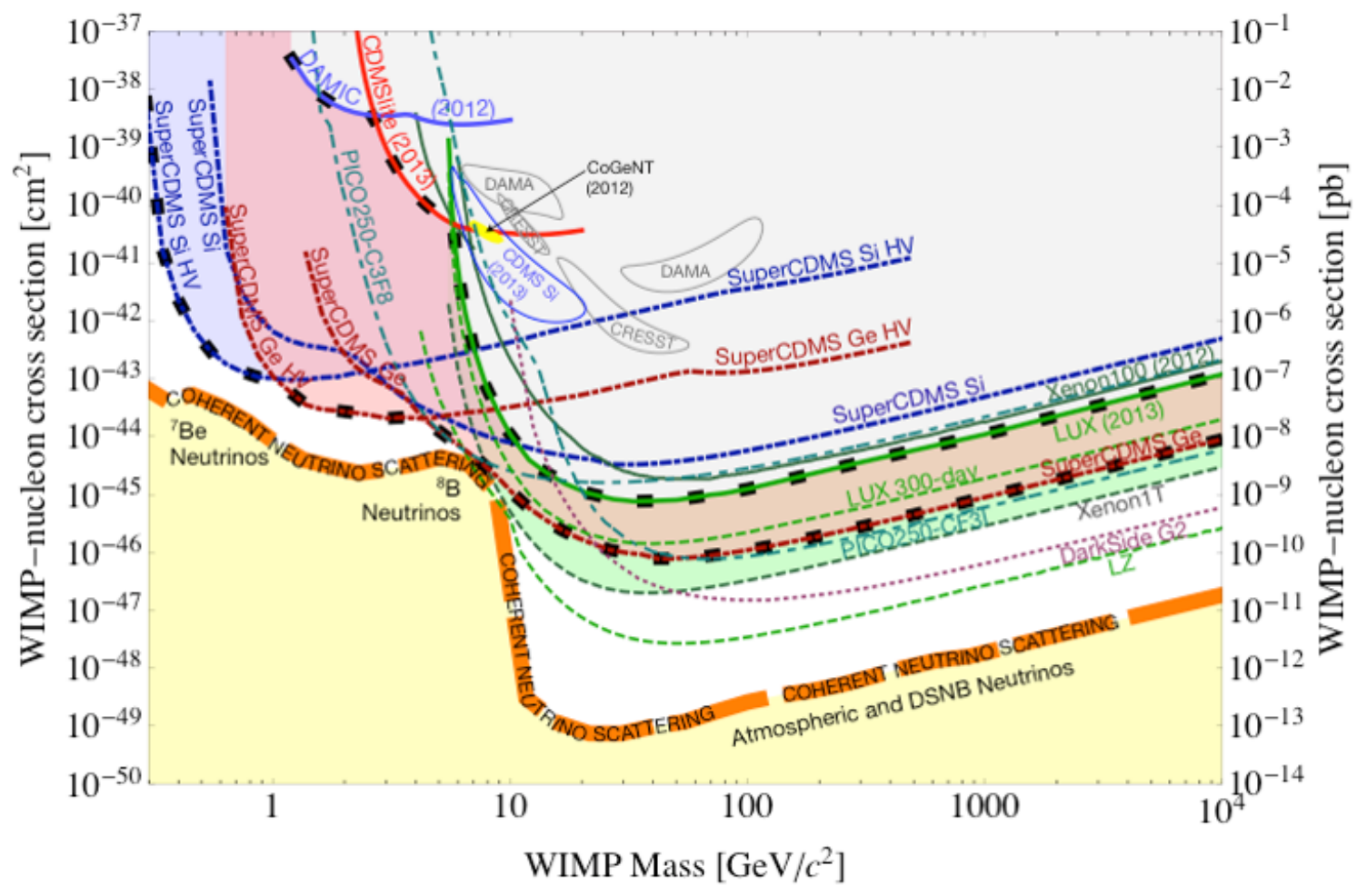

Figure 2.5: Limit plot showing results of previous experiments and project limits of future experiments.

\subsection{Results}

Historically, the CDMS experiment has been a leading technology in the direct dark matter search field. This remains true today as new evolutions in detector technology continue to push the limits (see Figure 2.5). As one of two approved direct search experiments (LZ being the other) for the next generation, the scientific community has again put its faith in this technology. The ability to operate at incredibly low energy thresholds keeps the CDMS experiment competitive in the low-mass dark matter search regime. LZ, having a much larger active mass, is able to lead the field in the push for lower interaction cross-sections. However, if either experiment claims a detection of dark matter, the scientific community will likely 
look to the other to confirm. In the case of an LZ discovery, CDMS would need to rapidly scale up mass to compete. This prospect is always under consideration in detector development, and may not be far out of reach in the near future. However, if CDMS finds evidence of a dark matter signature in the low mass region, it is unclear how or if LZ could accommodate such a search.

\subsection{Future}

The next generation of CDMS, SuperCDMS SNOLAB, is in the R\&D and design stage currently. It will incorporate Ge detectors $\sim 2.25 x$ as massive as SuperCDMS Soudan (see Figure 2.6) as well as incorporating silicon detectors into the payload to better target low mass WIMP recoils. Additionally, high voltage detectors will be implemented to utilize the voltage assisted calorimetric ionization detection measurement mentioned in section 2.4.2. 


\section{CDMS II}

\section{Single-sided \\ $1 \mathrm{~cm}$ thick \\ 3"diameter $250 \mathrm{~g} \mathrm{Ge}$}

2 charge +4 phonon

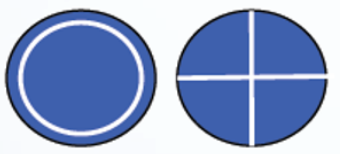

5 towers of 6 det each

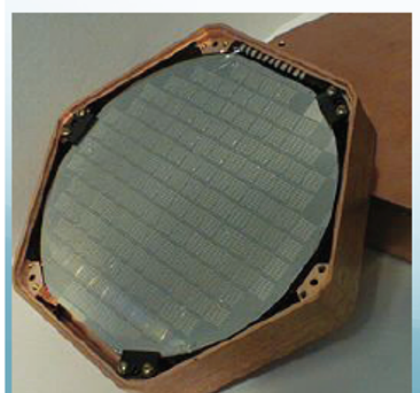

SuperCDMS Soudan

Double-sided $2.5 \mathrm{~cm}$ thick 3"diameter $620 \mathrm{~g} \mathrm{Ge}$

2 charge +2 charge

4 phonon +4 phonon
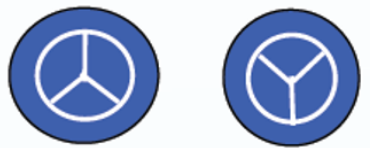

5 towers of 3 det each

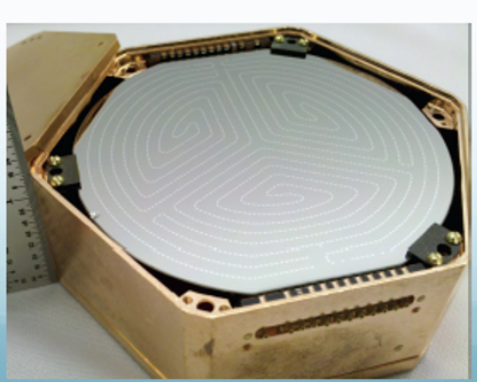

SuperCDMS SNOLAB

Double-sided

$3.3 \mathrm{~cm}$ thick

$100 \mathrm{~mm}$ diameter

$\mathrm{Ge}(1.4 \mathrm{~kg})$ or $\mathrm{Si}(0.6 \mathrm{~kg})$

2 charge +2 charge

6 phonon +6 phonon

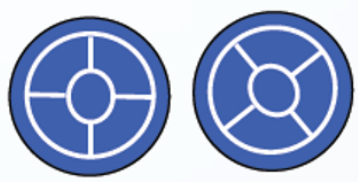

15 towers of 6 det each

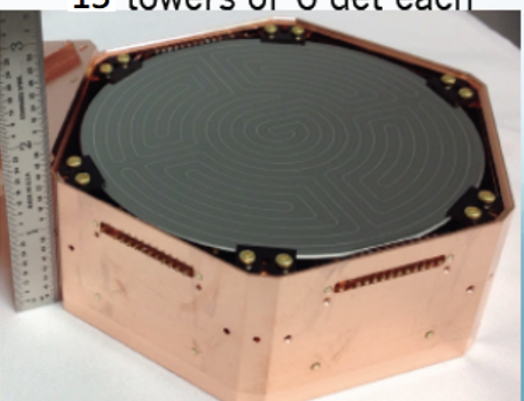

Figure 2.6: Evolution of CDMS experiments' detector size, payload, and sensor layout. 


\section{DETECTOR FABRICATION PROCESS AND IMPROVEMENTS*}

\subsection{Fabrication}

CDMS detectors are produced using techniques and equipment similar to those in typical semiconductor fabrication processes. The process begins with high quality semiconductor substrates (germanium or silicon) and uses photolithography to etch deposited films into circuit structures (see Figure 3.1). One significant difference, however, is that the substrates used for CDMS detectors are much thicker, ranging from $10 \mathrm{~mm}$ in original designs to $33.3 \mathrm{~mm}$ in current production. For this reason, semiconductor equipment and processing typically used for $\sim 1 \mathrm{~mm}$ thick substrates have been modified and tuned for these larger detector geometries.

\subsubsection{Substrate Materials}

Detectors are fabricated on high purity germanium and silicon substrates. For detector quality germanium substrates, "high purity" equates to impurity levels typically on the order of $10^{10} \mathrm{~cm}^{-3}$. These are grown using the Czochralski Process. Substrates used for this experiment typically have dislocation densities of $1000-7000 \mathrm{~cm}^{-2}$. For silicon detectors, quality is specified and determined by room temperature resistivity. While $>8 \mathrm{k} \Omega$-cm is the specification for acceptable material, typical detector quality substrates have a resistivity of $>20 \mathrm{k} \Omega-\mathrm{cm}$ and are grown using the Float Zone Process. All detectors currently operating in the SuperCDMS Soudan experiment are $76 \mathrm{~mm}$ diameter $\mathrm{x} 25 \mathrm{~mm}$ thick germanium substrates. The next generation will utilize both silicon and germanium detectors, $100 \mathrm{~mm}$ diameter by $33.3 \mathrm{~mm}$ thick[8].

\footnotetext{
*Reprinted with permission from "Cryogenic Dark Matter Search detector fabrication process and recent improvements" by Jastram et. al, 2015. Nuclear Instruments and Methods in Physics Research Section A: Accelerators, Spectrometers, Detectors and Associated Equipment, 772:14-25, Copyright 2015 by Elsevier
} 


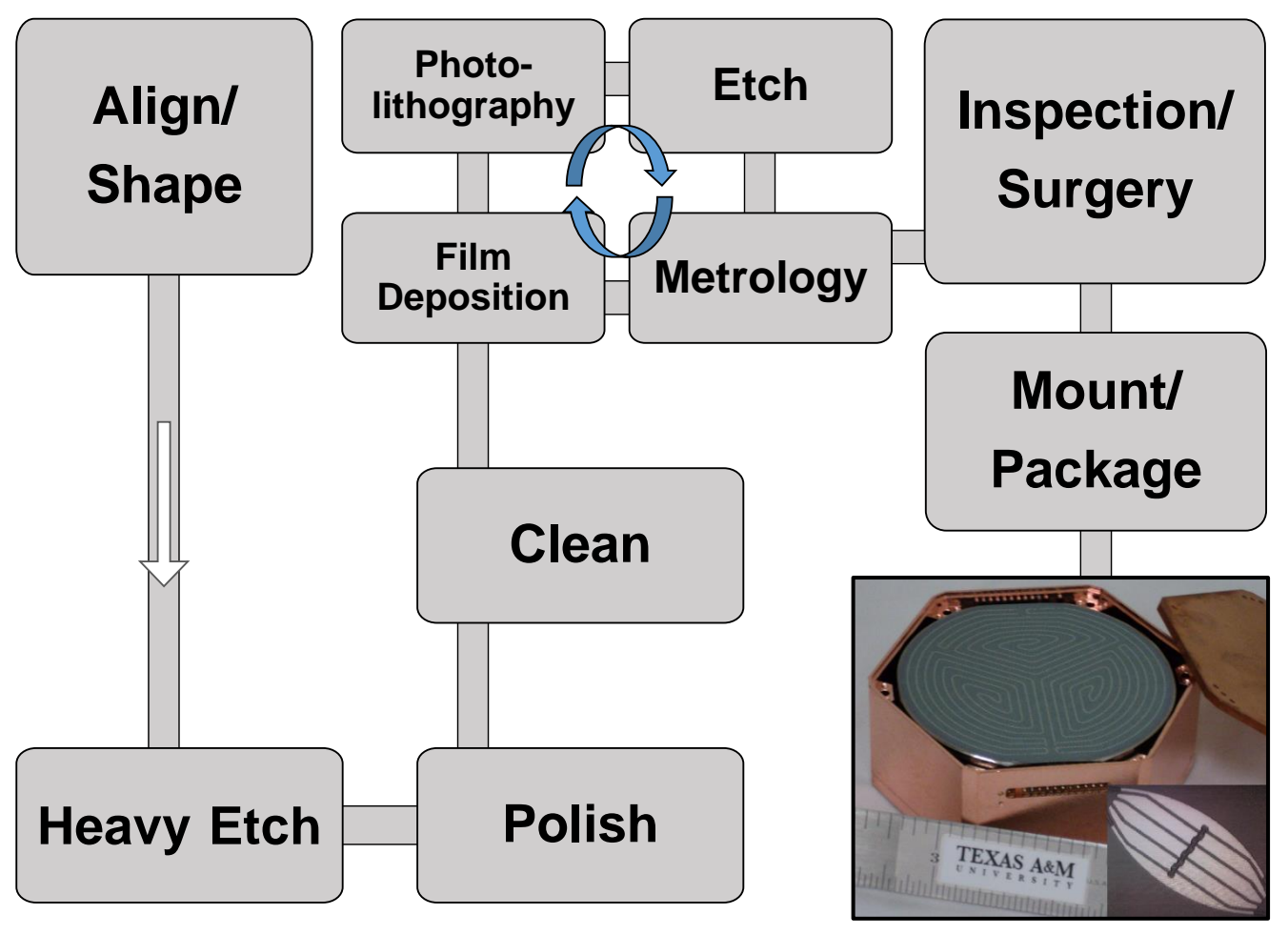

Figure 3.1: Process flow chart from raw substrate to completion (see Section 3.1).

The majority of this R\&D, including establishment and tuning of the fabrication process (specifically film characterization and photolithography steps), is performed using low resistivity commercial "Prime Grade" silicon wafers $(75 \mathrm{~mm}$ and $100 \mathrm{~mm}$ diameter with SEMI Standard ${ }^{1}$ thickness and flats). Being much lower in cost, easier to obtain, and easier to clean and prepare than thick substrates, they are a natural choice for practice and R\&D. Once established, fabrication procedures are then tested and confirmed on thick substrates. Low purity (and price) thick substrates are used for this before fabricating detector quality substrates.

\footnotetext{
${ }^{1}$ Standards and specifications available from www.semi.org
} 


\subsubsection{Alignment and Shaping}

To improve uniformity and charge collection performance among the detectors, the substrates are shaped and aligned to a specific crystal axis and orientation. Upon delivery from the vendor, the cylindrical substrates are guaranteed to be aligned within $\pm 2^{\circ}$ of the target crystal axis, typically [100]. For improved ionization drift and collection, they are subsequently re-shaped with the alignment refined to $\pm 0.1^{\circ}$. For this reason, the substrates, as purchased, are slightly over-sized in all dimensions to account for material loss in re-shaping. Re-shaping consists of aligning and grinding the substrates' faces, followed by grinding the cylindrical sidewall. A custom fixture has been made to allow the surface plane of the substrate to be manipulated with micrometers to precisely dial in the crystallographic axis to the coordinates of the x-ray diffractometer (XRD) used in this alignment process. For more information on the XRD process, see [9]. In this setup, a modified Rigaku DMAX-1BX is used with the x-ray source operated at $30 \mathrm{kV}$ and $20 \mathrm{~mA}$. First, the face of the substrate is positioned and aligned to the point of initial interference with the x-ray beam (which is set to $2 \theta=0^{\circ}$ ) with the face parallel to the beam and perpendicular to the goniometer's $\theta$ plane. The goniometer is then set to the Bragg angle of the target crystal axis and a local $2 \theta$ sweep is performed (the width of which is dictated by the alignment tolerance from the vendor). This produces a peak near the Bragg angle which will shift according to aforementioned micrometer adjustments. These adjustments and measurements are made iteratively (gross adjustments at first, followed by fine tuning) until the peak is within the required tolerance of the appropriate Bragg angle. The crystal is then locked into that orientation in the alignment fixture, which is designed such that it can be unmounted from the XRD system and attached to a

grinding fixture. This assembly is then placed on a Lapmaster 24C lapping machine 
(equipped with a 220 grit diamond magnetic plate) such that the substrate feeds into the grinding surface along the crystal axis. After grinding, this surface is measured again using XRD to confirm successful alignment. The second face is then ground parallel to the first using this same fixture. Parallelism of the faces is confirmed using

a granite indicator stand. The cylindrical sidewall must then be shaped parallel to the crystal axis. To reduce the chance of chipping during this process, circular plates of glass $(1 / 8$ " thick with a diameter 0.25 " larger than the final substrate diameter) are bonded to each face with a wax bonder using $69^{\circ} \mathrm{C}$ quartz wax. The sidewall shape is then defined using a diamond coring fixture. The coring diameter is that of the final substrate specification. The glass plates and quartz wax are then removed. To provide room for interface boards in the detector housings (see Section 3.1.8) and ensure all crystals are fabricated in a uniform rotational orientation, flats are ground on the sidewall of the substrate. These are located normal to a specific crystallographic direction ([011] in the case of [100] crystals). To perform this alignment, the crystal is loaded into a custom XRD mount with the previously aligned crystal axis normal to the $2 \theta$ plane and the x-ray beam incident upon the sidewall (with the sidewall now positioned to just slightly interfere with the beam while $2 \theta=0^{\circ}$ ). The goniometer is then set to the Bragg angle of the desired flat orientation, and the crystal is rotated about its axis (in the $2 \theta$ plane) using a precision rotary table indexer until the diffracted intensity is maximized (locating the orientation to $\pm 1^{\circ}$ ). Using a custom jig, the two diametrically opposed flats are ground using the Lapmaster 24C. The crystal is then lapped (on the same machine) to its desired thickness.

\subsubsection{Heavy Etch}

In order to remove substrate surfaces that may have been contaminated by previous processing and/or exposure to radon-containing atmosphere, the substrates 
are then chemically etched ${ }^{2}$. This process removes the outer layer (up to $\sim 250 \mu \mathrm{m}$ ) of material, which is assumed to be contaminated. Silicon substrate etching has not been used by CDMS in the past, but is currently under development for future detectors. Germanium etching is performed in the following solution:

\section{1. $3200 \mathrm{~mL} 69 \% \mathrm{HNO}_{3}$}

2. $640 \mathrm{~mL} 50 \% \mathrm{HF}$

\section{3. $150 \mathrm{~mL} \mathrm{CH}_{3} \mathrm{CO}_{2} \mathrm{H}$ (glacial)}

The substrate is dipped in the etchant using a modified PTFE wafer cassette (used in all subsequent acid processing) and agitated lightly by hand, followed by a dip in de-ionized (DI) water. This is repeated 10 times. It is then placed in a Verteq 1600$55 \mathrm{M}$ spin rinse/dryer for a standard rinse/dry process (to be referred to as SRD). The SRD process consists of the following steps:

1. 35 seconds @600rpm with $\mathrm{N}_{2}$ purge and DI spray

2. 230 seconds @1600rpm with heated $\mathrm{N}_{2}$ purge

3. 90 seconds @1600rpm with $\mathrm{N}_{2}$ purge

Following this step, substrates are stored in nitrogen purged cabinets when not being actively processed, reducing subsequent exposure to ambient radon.

\subsubsection{Lapping and Polishing}

Photolithographic processing of micron scale features requires a smooth, featureless substrate surface. For this reason, the coarsely lapped, heavy-etched detector faces must be polished. This is accomplished via four sequential steps:

\section{Fine-grit manual lapping}

\footnotetext{
${ }^{2}$ This etch recipe is based on a process described in [10] modified by Paul Brink and Larry Novak.
} 
2. Surface shaping polish

3. Scratch-removing polish

4. Final surface treatment polish

Substrates are hand-lapped on a slotted glass lapping plate using $9 \mu \mathrm{m}$ alumina polishing powder mixed with DI water to form a paste consistency. This is to remove large features from the surface. The surfaces must then be polished to a specified flatness with a mirror finish, free of visible features (such as scratches or pits) to facilitate uniform film depositions and prevent circuit defects (see Section 3.1.8) in subsequent processing. Polishing is performed on a dual spindle polishing machine. Control of surface curvature (concavity vs. convexity) is maintained with polisher settings and various sizes of polishing pads surfaced with polyester material in a 1:1 mixture of colloidal alumina polishing compound:DI water. This process is carefully controlled such that the final surface has $<2 \mu \mathrm{m}$ of total height deviation across the substrate if convex, $<1 \mu \mathrm{m}$ if concave (curvature is measured with a desktop laser interferometer). This is to ensure uniform contact with the photo mask (which can conform slightly to convexity but not concavity) during the photolithography process. Small surface scratches resulting from this step are then removed on the same machine using "regular nap" polyurethane pads and a fresh mixture of the same polishing slurry. Final surface polishing is performed with "high nap" polyurethane pads in a colloidal silica polishing compound. Final surface inspection is performed using a stereo zoom binocular microscope, manually confirming a defect free mirror finish.

\subsubsection{Cleaning}

Before the polished substrates can be processed into detectors, they must be cleaned carefully. This removes surface contaminants as well as any particulates that 
may cause defects in subsequent processing (see Section 3.1.8). For this reason, the cleaning is performed in a class 100 clean room. Germanium and silicon substrates require different cleaning processes, germanium's being much more time and labor intensive (another benefit of using silicon wafers for R\&D).

\subsubsection{Germanium}

Initial germanium cleaning involves a 5 minute soak in acetone followed by a 5 minute soak in isopropyl alcohol (NOTE: all chemicals used in cleaning and subsequent processing are semiconductor grade). Following a thorough rinse with DI water, the surfaces are manually inspected using a microscope equipped with an LED ring light (especially effective for identifying particles on the surface as it exposes diffuse features). If particulate count is unacceptable $\left(\geq 10 \mathrm{~cm}^{-2}\right)$, the previous chemical process is repeated, and the crystal is dried using a filtered nitrogen gun. If particulate count is still unacceptable, the substrate is rinsed with methanol and manually wiped with a PVA cleaning brush. If the surface condition is still unacceptable, the methanol and brush wipe is repeated as necessary. Otherwise, the substrate proceeds to the oxide removal step. For this, a mixture of 3:1 DI water:50\%HF is prepared in which the substrate is submersed for 5 minutes followed by a 3 minute soak in DI water. This is repeated three times and followed by surface inspection. If particulate count has become unacceptable, a methanol rinse and brush wipe are repeated as necessary. Upon completion, the substrate is placed in the oven at $120^{\circ} \mathrm{C}$ for 10 minutes to bake out remaining moisture.

\subsubsection{Silicon}

Silicon substrates also receive chemical cleaning, but have not shown the need for manual particulate removal. In the cleaning process, the substrates are initially doused with methanol then isopropyl alcohol, followed by SRD. To remove metals 
and organic contaminants, a Piranha clean process is used. This consists of a 20 minute dunk in the following solution (heated to $55^{\circ} \mathrm{C}$ ):

1. $600 \mathrm{~mL} 30 \% \mathrm{H}_{2} \mathrm{O}_{2}$

2. $700 \mathrm{~mL} 98 \% \mathrm{H}_{2} \mathrm{SO}_{4}$

3. $1050 \mathrm{~mL} 0.250 \mathrm{~N} \mathrm{H}_{2} \mathrm{SO}_{4}$

Substrates are then soaked in $55^{\circ} \mathrm{C}$ DI for 1 minute, manually agitated once every 15 seconds. To remove the native oxide from the substrates' surfaces, they are then placed in the following solution for 20 seconds:

1. $2700 \mathrm{~mL} \mathrm{DI}$

2. $50 \mathrm{~mL} 50 \% \mathrm{HF}$

The substrates are then dipped again in $55^{\circ} \mathrm{C}$ DI for 1 minute, manually agitated once every 15 seconds. To remove ionic and heavy metal atomic contaminants from the substrates' surfaces, the substrates are submersed in the following solution, heated to $70^{\circ} \mathrm{C}$, for 15 minutes:

1. $1750 \mathrm{~mL}$ DI

2. $325 \mathrm{~mL} 30 \% \mathrm{H}_{2} \mathrm{O}_{2}$

3. $300 \mathrm{~mL} 37 \% \mathrm{HCl}$

This is followed by SRD and a 5 minute dehydrate in the oven at $120^{\circ} \mathrm{C}$. The cleaning process seals the substrate with a thin oxide layer which is removed in the sputtering system prior to film deposition (see Section 3.1.6).

\subsubsection{Thin Film Deposition and Tuning}

The films that form the final circuit and sensors of the detector are deposited using a customized plasma sputtering deposition system. Precise and repeatable 

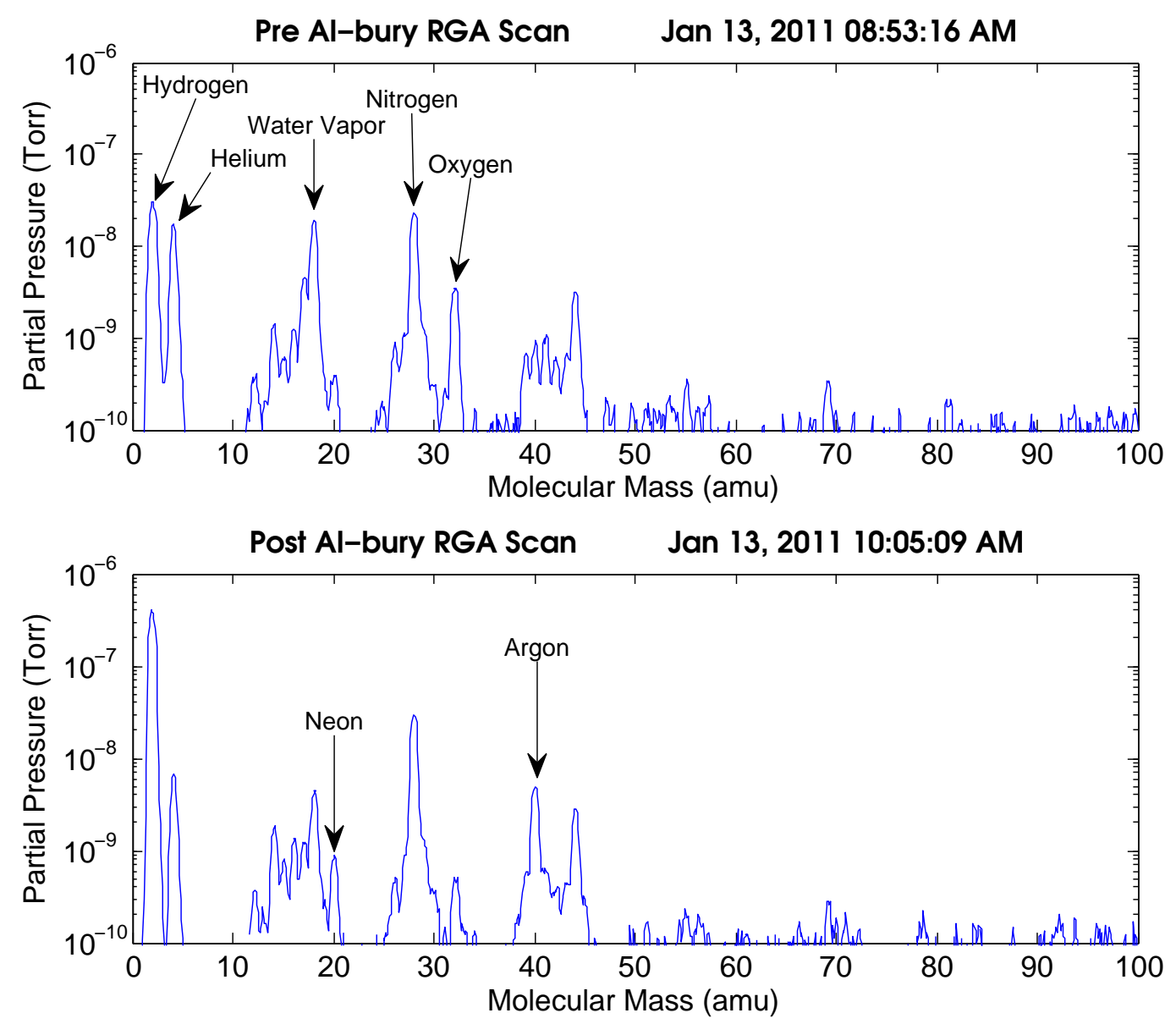

Figure 3.2: Example RGA spectra of partial pressures in the SEGI process chamber at various atomic masses before (top) and after (bottom) aluminum getter deposition (see Section 3.1.6), demonstrating the efficacy of this process in reducing oxygen and water vapor levels. 
process control is vital in the deposition of these films as they dictate the quality of the final circuit features (see Sections 3.2.1, 3.2.2, and 3.2.3). The deposition system used in this process is a Perkin Elmer 4400 Delta with PLC/PC interface automated by Semiconductor Engineering Group, Inc. (SEGI), which has been modified for this fabrication process. It can simultaneously sputter 8 substrates, $100 \mathrm{~mm}$ in diameter (or $6,150 \mathrm{~mm}$ diameter) and is composed of two main vacuum chambers: the load lock and the process chamber, separated by a gate valve. The load lock is the location in which substrates are initially loaded, which is then pumped to $4.0 \times 10^{-6}$ Torr using a turbo-molecular pump (added for this process). This allows the process chamber to stay isolated from atmospheric contaminants at all times (specifically when the substrate is transferred through the gate valve into this chamber). To further reduce contaminants, a pre-coat of Aluminum is sputtered in the process chamber as a getter. This process removes traces of $\mathrm{O}_{2}$ and $\mathrm{H}_{2} \mathrm{O}$ (see Figure 3.2) as well as other contaminants which can alter film characteristics, further improving process stability. The substrates are then transferred into the process chamber, which is subsequently pumped back to a base pressure of $9.0 \times 10^{-7}$ Torr. The process chamber is cylindrical ( $\sim \mathrm{m}$ in diameter) and can simultaneously accommodate three different targets of sputtering materials. The chamber is equipped with aluminum, tungsten, and silicon targets, all of which are $99.999 \%$ pure. After substrates are loaded in the load lock, the rest of the deposition process is entirely automated, including everything from rotation and height settings of the table on which the substrates sit to setting and maintaining the chamber and plasma conditions. Table height settings are customized for each substrate thicknesses to maintain a constant target-substrate distance. Other settings of particular note are the voltage and power supplied to the target (DC or RF), DC bias applied to substrates during deposition, flow of Argon into the chamber, and optional RF pre-etch. 


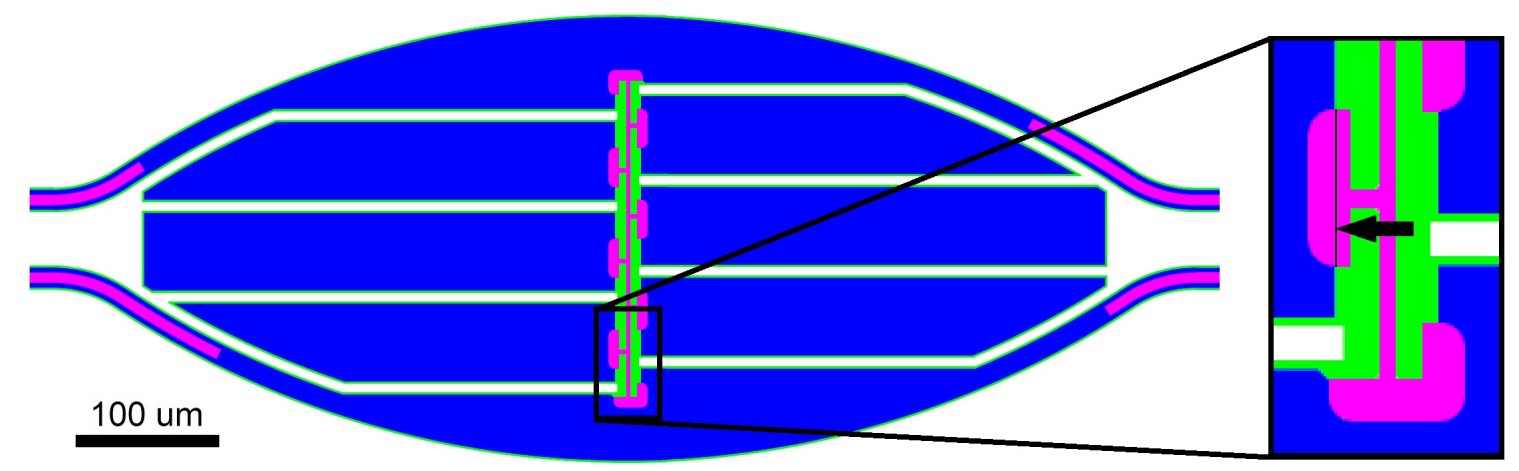

Figure 3.3: Image of a single phonon sensor and magnified inset of TES line and "waterfall" region (arrow indicates waterfall boundary, see Section 3.2.3 for description). Each color corresponds to the exposed film on the final structure: Blue=Aluminum (mask \#1, trilayer mask), Pink=Tungsten (mask \#2, TES mask), Green=a-Si (mask $\# 3$, "trench" mask) (see Section 3.1.7). The central vertical line is the $\sim 2 \mu \mathrm{m}$ wide TES, and the large aluminum "fins" are the phonon absorbing structures (see Section 3.2.1). Note: a-Si underlies all metal features.

The process chamber is equipped with an RGA (Residual Gas Analyzer) which measures the contents of the gas in the process chamber. The RGA can be used in two modes: plotting the entire spectrum at once, showing peaks at various masses corresponding to contaminants in the chamber (see Figure 3.2), or plotting the levels of a chosen contaminant over time. A high capacitance valve is implemented to allow the RGA to operate at high vacuum levels as well as in-process levels ( $\sim 10 \mathrm{mTorr})$. This allows in-situ analysis of any possible gas contaminants during the deposition process.

All of the above devices and processes allow the minimization of contaminants and maximization of control and repeatability in the deposition process. Using this system, three thin film layers (designated as the trilayer) are sputtered sequentially on both faces of each substrate: 40nm amorphous silicon (a-Si), 300nm aluminum, and 30nm tungsten. The a-Si layer underlies all final metal circuit structures and is used to protect the substrate surface from aluminum and tungsten etchant chemicals, as 
well as improving the ionization collection boundary. The aluminum layer forms the phonon collection structures (see Figure 3.3 and Section 3.2.1) as well as the circuit lines (electrodes) connecting the sensors on the detector. The tungsten layer serves as a cap layer, preventing the aluminum surface from oxidizing (see Section 3.2.3) and preventing back-sputtering of the aluminum during the subsequent deposition. Before each deposition begins, the target to be used is pre-sputtered for 25 seconds to clean its surface with its shutter closed (to prevent sputtered material from depositing on substrates). Before all depositions, an aluminum shadow mask is manually placed on the substrate face which covers the outer $\sim 1 \mathrm{~mm}$ of the exposed surface, preventing deposition on this region (see Section 3.2.5) and the substrate's sidewall surface. The steps and parameters used for the trilayer deposition are the following:

1. 10 minute RF etch, $350 \mathrm{~W}$ RF, 50 sccm Ar, 10 mTorr

2. 16 minute a-Si deposition, $500 \mathrm{~W}$ RF, 50 sccm Ar, 8 mTorr

3. $7 \mathrm{~m} 18 \mathrm{~s}$ aluminum deposition, $2.5 \mathrm{~kW}$ DC, $40 \mathrm{sccm}$ Ar, 10 mTorr

4. 36 second tungsten deposition, $2.5 \mathrm{~kW}$ DC, 40 sccm Ar, 8 mTorr

The films are then patterned photolithographically and chemically etched, forming the majority of the detector circuit (described in Section 3.1.7). After chemical etching, a 40nm layer of tungsten is sputtered on each face of the substrate. This layer forms the transition edge sensors (TES's) of the detector (see Sections 3.2.2 and 3.2.3). The steps and parameters used for this deposition are as follows:

1. 10 minute RF etch, $350 \mathrm{~W}$ RF, 50 sccm Ar, 10 mTorr

2. 51 second tungsten deposition, $2.5 \mathrm{~kW}$ DC, 40 sccm Ar, 8 mTorr, 100V DC bias delivered to substrate

This layer requires the most precise tuning and consistency, described in Sections 3.2 .2 and 3.2.3. 


\subsubsection{Photolithography}

A three step photolithographic process (see Figure 3.4) is used to define the circuit features on the substrates. The original process from which this was adapted is described in [11] and [12]. The first step defines the aluminum structures (circuit lines and phonon collecting fins [see Section 3.2.1]). The second step defines the tungsten TES features (see Section 3.2.2), and the third defines the a-Si structure and substrate trenching regions (see Section 3.2.4). The aluminum and tungsten are etched with chemicals, while the a-Si is plasma etched via an RIE (Reactive Ion Etch) process. In all three steps, an etch resistive mask of photoresist is used to protect the features while the exposed films are etched. The photoresist mask pattern is formed via UV transfer (exposure) using a master template mask. Chemical processing of the substrates is performed in a class $100 \mathrm{UV}$-free clean room.

\subsubsection{Trilayer Patterning}

After the trilayer deposition (see Section 3.1.6), a Solitec 5110-SJ spin coater is used to spin coat Shipley Microposit S1811 photoresist on both faces of the substrate. To create and maintain a vacuum seal between the substrate and spin coater's chuck, 0.032" thick elastomer skirts are stretched around the substrate sidewall. These are removed and discarded after the spin coat process (see Section 3.2.5 for more information on the photoresist layer and process). The substrate is then placed in the oven (in a PTFE cassette, covered with aluminum foil to protect the fresh

photoresist from particulates) at $120^{\circ} \mathrm{C}$ to soft bake the photoresist (see Table 3.1 for bake times).

After baking, the crystal is allowed to cool to room temperature. The cooling process is accelerated with a gentle stream of filtered nitrogen gas on each face. The substrate is then ready for the photolithographic mask transfer (exposure) process. 

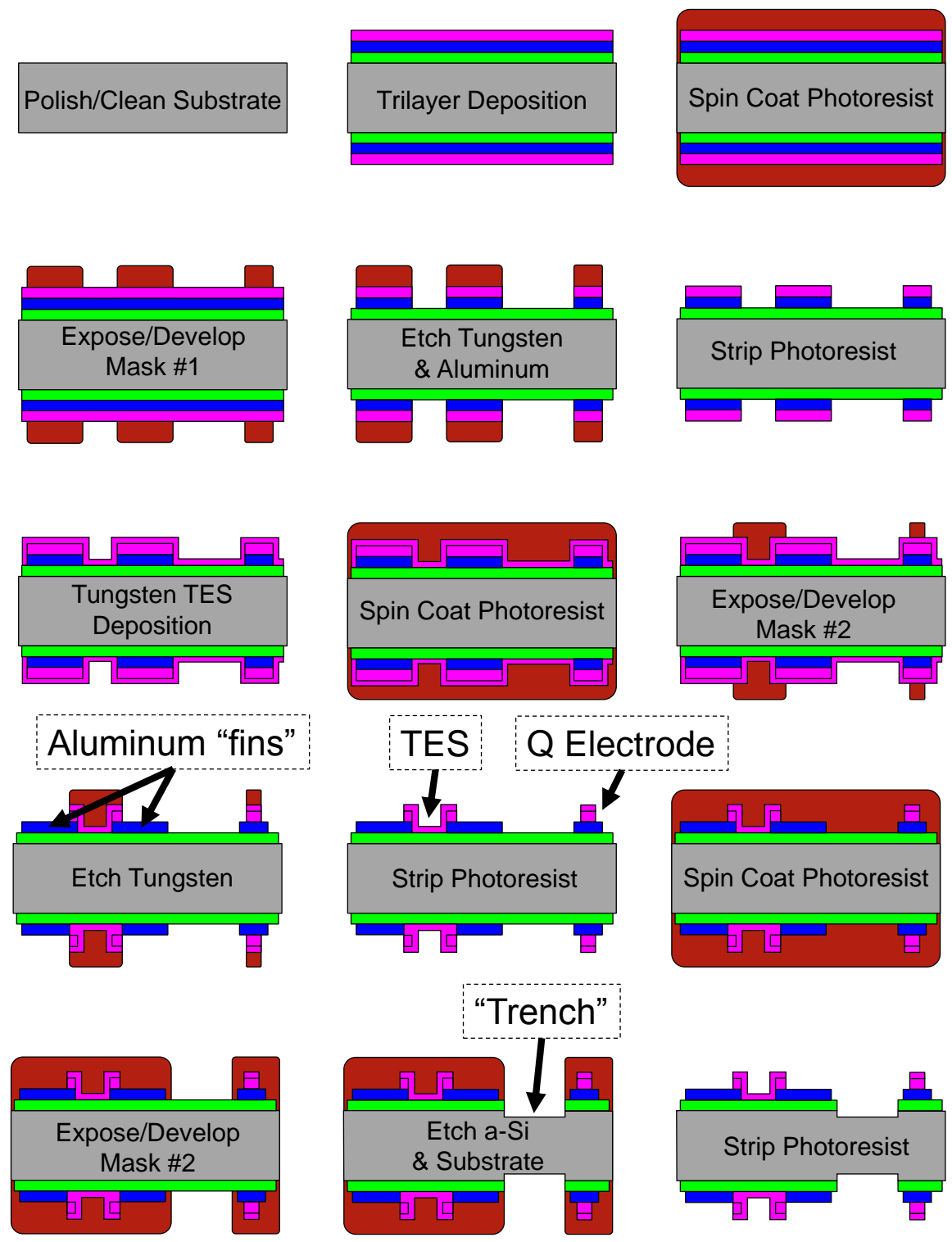

Figure 3.4: Detector patterning process (not to scale). Individual film layers are shown throughout the deposition and photolithography process (see Section 3.1.7). Gray $=$ Substrate, Green $=\mathrm{a}-\mathrm{Si}, \mathrm{Blue}=$ Aluminum, Pink=Tungsten, and Redbrown $=$ Photoresist. 

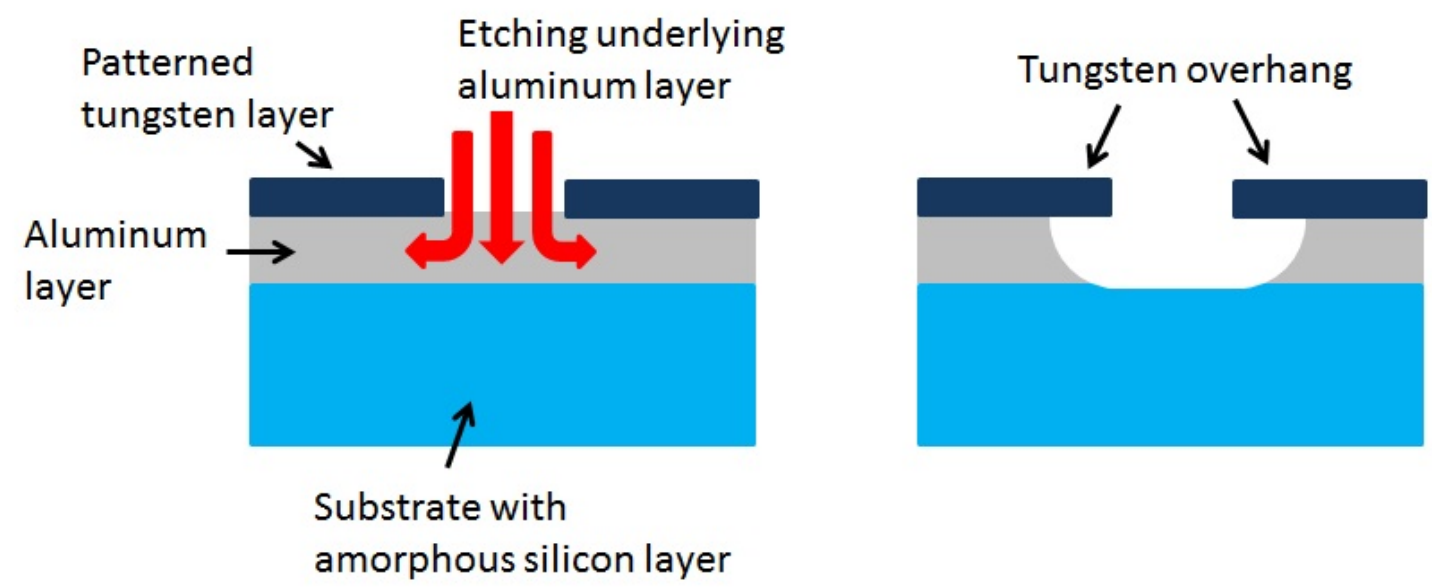

Figure 3.5: Diagram depicting the tungsten overhang issue caused by the isotropic aluminum etch process (not to scale). Figure reprinted with permission from [13].

\begin{tabular}{|c||c|c|}
\hline Size (Dia. $\times$ Thickness) & Soft Bake & Hard Bake \\
\hline $76 \mathrm{~mm} \times 10 \mathrm{~mm}$ & $20 \mathrm{~m}$ & $1 \mathrm{~h} 30 \mathrm{~m}$ \\
$76 \mathrm{~mm} \times 25 \mathrm{~mm}$ & $25 \mathrm{~m}$ & $2 \mathrm{~h}$ \\
$100 \mathrm{~mm} \times 33.3 \mathrm{~mm}$ & $28 \mathrm{~m}$ & $2 \mathrm{~h} 20 \mathrm{~m}$ \\
\hline
\end{tabular}

Table 3.1: Bake times for various substrate sizes. Thin (practice) wafers soft bake for $1 \mathrm{~m} 50 \mathrm{~s}$ on a $115^{\circ} \mathrm{C}$ hot plate, and hard bake for 15 minutes in the oven at $120^{\circ} \mathrm{C}$. 
An OAI 206-094735 contact aligner with a 350W Hg g-line UV lamp is used to expose each face for $5.3 \mathrm{~s}$ at $8.15 \mathrm{~mW} / \mathrm{cm}^{2}$ using mask \#1, the trilayer mask (see Figure 3.3). Special care must be taken not to scratch the backside photoresist layer when placing the substrate on the stage (and when flipping the substrate for exposure of the second face). The UV intensity is confirmed before each exposure using an OAI 0308 UV meter tuned to 436nm (g-line). The pattern is then developed using Shipley Microposit MF-319 developer, mildly agitated by hand, until completion. This is judged by eye, typically taking 70-90 seconds. The substrate is gently rinsed in DI water after development, then proceeds to SRD. The photoresist is then inspected to confirm successful development (robust replication of mask structure). The substrates are then returned to the oven at $120^{\circ} \mathrm{C}$ to hard bake the photoresist (see Table 3.1 for bake times). They are then cooled to room temperature with the assistance of gentle nitrogen gas flow. The tungsten layer is etched for 6m30s using $30 \% \mathrm{H}_{2} \mathrm{O}_{2}$ with 2 gentle manual agitations at 1 minute intervals, followed by SRD. The aluminum layer is etched using Cyantek Al-11. This typically consists of 5-6 iterations of the following: 45s Al-11 dunk with constant gentle agitation, followed by a 15s DI rinse. The aluminum etch leaves a slight overhang of the tungsten cap layer, due to the isotropic nature of the reaction (see Figure 3.5). Intermittent DI rinses are used to control the temperature of the exothermic etch reaction[14], improving etch uniformity and reducing the undercut (overhang) issue. When all exposed aluminum appears to have vanished, the substrate receives an additional 15s of Al-11 etch to ensure no aluminum remains, then proceeds to SRD. To remove the overhang feature, another tungsten etch is performed (see Section 3.2.3). This consists of a 10 minute submersion in $30 \% \mathrm{H}_{2} \mathrm{O}_{2}$, with 2 gentle agitations every 2 minutes, followed by SRD. At this point, the circuit pattern is carefully inspected to confirm successful etching and preserved photoresist integrity. The photoresist layer is removed using 
a 20 minute dip in Shipley PRX-127 at $45^{\circ} \mathrm{C}$, with 2 gentle agitations every 5 minutes, followed by SRD. As a final cleaning precaution, the substrate is submerged in Baker PRS-1000 for 10 minutes at $45^{\circ} \mathrm{C}$, followed by SRD. Etched features are then inspected (and again after each subsequent photolithography cycle), monitoring for defects and critical circuit feature dimensions. The substrate is then placed in the SEGI under vacuum overnight to boil off any moisture before the following tungsten (TES layer) deposition.

\subsubsection{TES Patterning}

The second deposition, that which forms the TES tungsten layer, is then performed (see Section 3.1.6). After this deposition, the substrate receives the same spin coat, soft bake, alignment, exposure, develop and hard bake process as previously mentioned. The mask used for this layer (mask \#2, see Figure 3.3) defines the TES structures on the circuit. After hard bake and cooling, the tungsten is etched in $30 \% \mathrm{H}_{2} \mathrm{O}_{2}$ for 12 minutes, with 2 gentle agitations every 2 minutes, followed by SRD. It should be noted that this step etches all tungsten not covered by the mask, including the tungsten cap layer from the mask \#1 structures. Therefore, anywhere that masks \#1 and 2 coincide, all four film layers remain. Otherwise, mask \#2 defines structures with only TES tungsten on top of a-Si (see Figure 3.4 and Section 3.2.3). The photoresist is then inspected for integrity and removed with the same PRX-127 and PRS-1000 process as before (aside from PRX-127 time reduction to 15 minutes). The substrate is then placed under vacuum overnight to remove moisture (improving adhesion of subsequent photoresist coat).

\subsubsection{3 a-Si Patterning and "Trenching"}

The last photolithography step defines the a-Si structure with mask \#3, using the same spin coat, soft bake, alignment, exposure, develop, hard bake, and cooling 
process as previous steps. After hard bake and cooling, the a-Si is etched in a modified Tegal 903C reactive ion etcher using 8 iterations of the following etch and cool down steps:

1. 18 second etch, $400 \mathrm{~W}$ RF @ $13.56 \mathrm{MHz}, 18$ sccm SF6, 50 sccm He, 1100 mTorr

2. 7 minute purge (cool down), $50 \mathrm{sccm}$ He, 900 mTorr (limited by Helium MFC)

See Section 3.2.4 for more information on this etch. The photoresist is then removed with the same PRX-127 and PRS-1000 process as before (with original PRX-127 time of 20 minutes).

\subsubsection{Inspection, Surgery, and Mounting}

It is possible for defects to arise in the photolithography process which can prevent a detector from operating as desired. For this reason, every element of every detector circuit is manually inspected using a microscope. This step is crucial to successful detector fabrication as micron scale defects can knock out an entire sensor channel. Defects of concern include areas of missing metal, causing breaks in the circuit continuity as well as metal films that did not etch properly, causing shorts (see Figure 3.6). In the case of open circuits due to breaks in metal continuity, a Kulicke \& Soffa 4523AD wire bonder (with a DewyL Tool MCSOE-1/16-750-45-C2025-M wedge and $0.00125 "$ diameter $99 \% \mathrm{Al} \mathrm{1 \%} \mathrm{Si}$ wire) is used to connect the isolated metal regions with wire bonds. The circuit is designed with extra metal pads (bonding locations) to make this task easier. In the case of a defect causing a short circuit, repairs can be made using one of two options:

1. Manually abrading the film with the wedge of the wire bonder to eliminate the unwanted electrical connection (an auxiliary wedge should be used for this, preventing damage to the bonding wedge) 
2. Using a localized droplet of the proper chemical etchant to remove the metal. This can also be used during the photolithography process (depending on the nature and location of the defect), utilizing the protective photoresist mask, thus minimizing chemical damage to nearby structures.

The detectors are then mounted in hexagonal OFHC copper housings which include Detector Interface Boards (DIBs). The detector electrodes have large bond pads near the DIBs, used to wire bond the detector channels to copper traces on the DIBs. This provides a feed-through to the outside of the grounded housing as well as a rigid connection to external hardware.

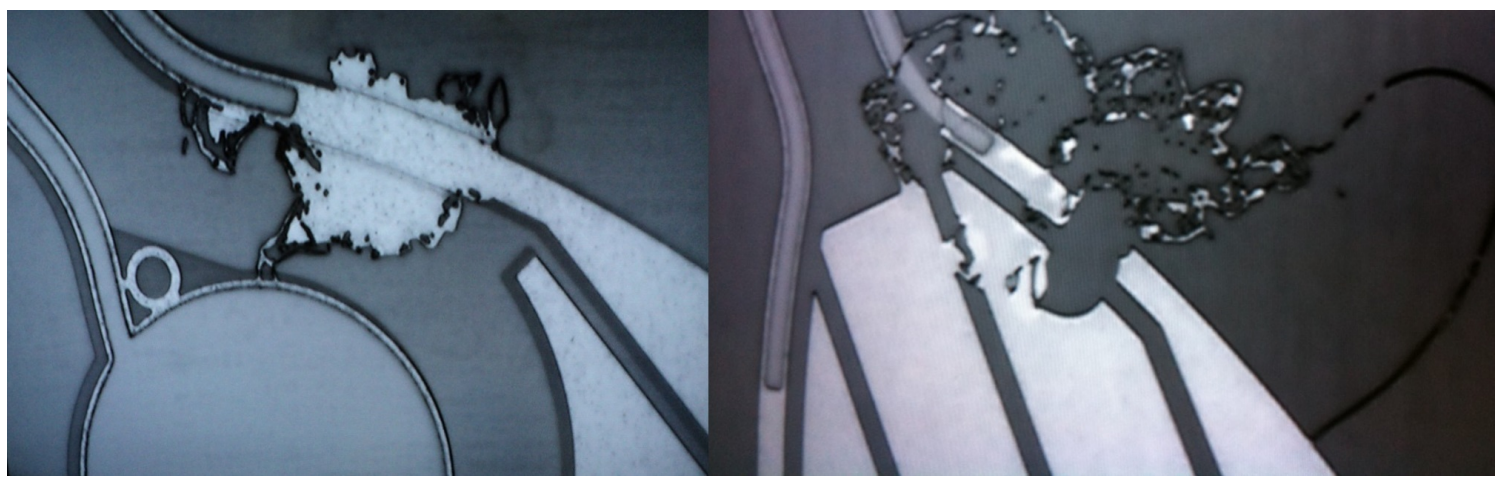

Figure 3.6: Left) Example of an un-etched section of Aluminum, causing a possible short. Right) Example of photoresist failure allowing unwanted aluminum etching, breaking circuit continuity, and requiring a "surgery" wire bond. Both examples were likely caused by particulates on the detector surface during early processing.

\subsection{Process Tuning, Results, and Improvements}

\subsubsection{Aluminum Film}

To efficiently read out phonon energy, the phonons are first absorbed in the superconducting aluminum "fins" (see Figure 3.3) where the energy is used to dissociate 
Cooper pairs which split into pairs of quasi-particles. These quasi-particles must diffuse through the aluminum to reach the tungsten TES where they are essentially trapped due to the tungsten's lower superconducting bandgap value (see Section 3.2.3). A crucial property of the aluminum is a high quasi-particle diffusion length. This length is inhibited by impurities found in the aluminum.

Quasi-particle diffusion length is important to monitor and control. To quantify the quality of the aluminum film, a measurement of the residual resistivity ratio (RRR) is performed. This is the ratio of the film's resistivity at room temperature to its resistivity at $4 \mathrm{~K}$. A higher RRR value corresponds to a higher quality film (i.e. one with fewer impurities) [15]. A RRR value of 10 has been deemed sufficient for a well functioning device in these detectors, but films have been tuned using the SEGI to routinely achieve a value of $\sim 16$.

\subsection{2 $T_{c}$ Tuning}

Optimal detector readout relies on the TES sensors being held at specific temperatures in their superconducting-transition resistance curves. The second tungsten deposition forms the TES layer. Consequently, this deposition must be carefully tuned to produce tungsten of a uniform, consistent, and precisely-controlled critical temperature $\left(\mathrm{T}_{c}\right)$. Critical temperatures of thin tungsten films are largely dictated by the ratio of $\alpha$ to $\beta$ phase in the material. This is due to the fact that the $\alpha-\mathrm{W}$ exhibits a $\mathrm{T}_{c}$ of $15 \mathrm{mK}$ [16] while $\beta$-W can have $\mathrm{T}_{c}$ 's ranging from 1 to $4 \mathrm{~K}$ [17]. Utilizing this and the fact that the two phases have different crystallographic structures (and therefore, different Bragg angles), provides a technique of roughly estimating the $\mathrm{T}_{c}$ of a given sample at room temperature using XRD[17] (see Figure 3.7). This technique is useful for tuning film samples to have high $\alpha: \beta$ ratios $\left(\mathrm{T}_{c}\right.$ 's closer to the desired range), but in this range, the ratio becomes so heavily $\alpha$-dominated 

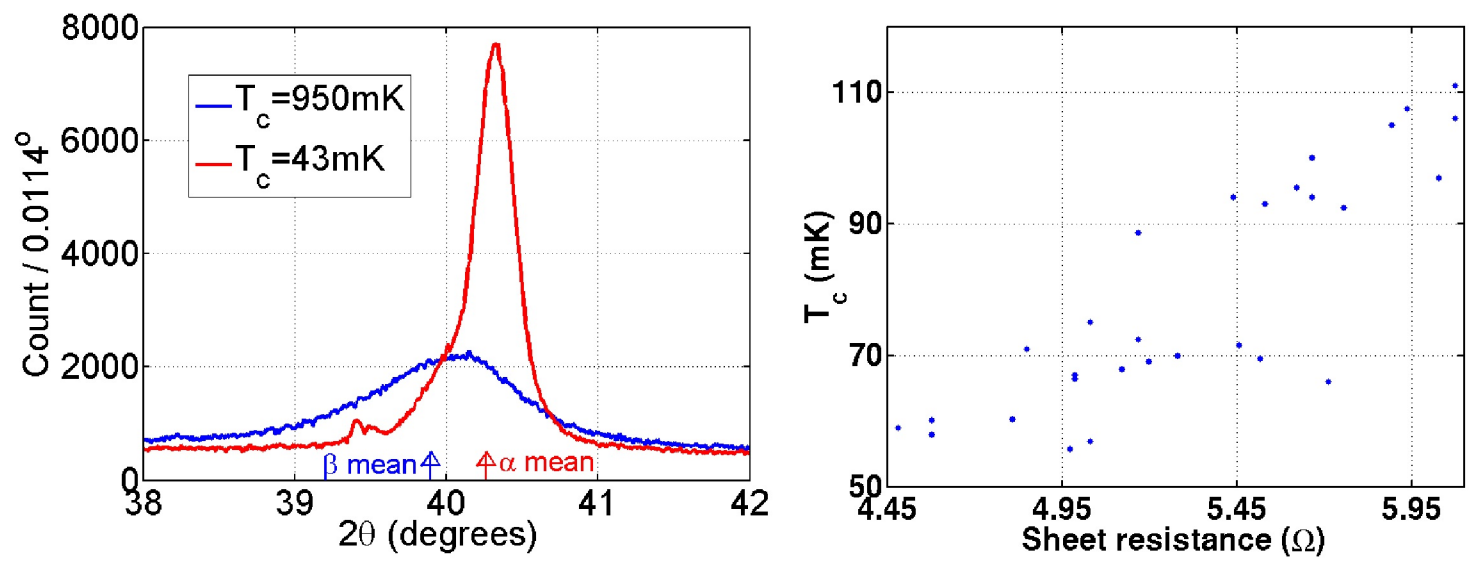

Figure 3.7: Left) XRD spectra showing discrimination between samples of differing $\alpha: \beta$ concentrations and their resulting $\mathrm{T}_{c}$ 's. Also marked are the locations of the peaks of pure $\alpha$ and $\beta$ phase films. An algorithm fitting two Gaussian functions (centered at these values) provides an estimate of phase ratios, and therefore $\mathrm{T}_{c}$ 's, of the films. Right) Plot showing correlation of critical temperature vs. sheet resistance of similar thickness films $(40 \pm 4 \mathrm{~nm})$ [13]. These room temperature characterization methods allow film deposition parameters to be tuned without the time or monetary expense of dilution refrigerators (see Section 3.2.2). Reprinted with permission.

that differences in $\beta$ concentrations become indistinguishable, making $\mathrm{T}_{c}$ predictions difficult. To finely tune deposition parameters to the $\sim 80 \mathrm{mK}$ target, a dilution refrigerator is used to physically measure the resistance transition as the sample is cooled past its $\mathrm{T}_{c}$ and again as it warms up. With this feedback, depositions with different sputtering power, substrate bias, and argon pressure were produced and tested, creating films of varying $\mathrm{T}_{c}$ 's. In this process, a correlation was established connecting room temperature resistivity of the films to their $\mathrm{T}_{c}$ (see Figure 3.7, right), allowing recipes to be roughly tuned and chosen with simple room temperature measurements (sheet resistance measured with a 4-point probe, corrected for film thickness to calculate resistivity)[17]. Using these processes, a recipe was chosen to produce films possessing the desired $\mathrm{T}_{c}$. Current experimentation with devices of varying $\mathrm{T}_{c}$ 's rely 
heavily on the resistivity- $\mathrm{T}_{c}$ correlation, saving significant time and money required for dilution refrigerator tests.

In addition to depositing films with carefully tuned, repeatable $\mathrm{T}_{c}$ 's, the SEGI has demonstrated the ability to produce films with much higher $\mathrm{T}_{c}$ uniformity across the substrate surface. Previous systems have had large $\mathrm{T}_{c}$ gradients across the face of detectors, beyond an acceptable limit (see Figure 3.8). To correct this issue, $\mathrm{T}_{c}$ distributions must first be mapped (requiring detector testing in a dilution refrigerator), followed by ion implantation of ${ }^{56} \mathrm{Fe}$ (specifically into the TES's) to correct for the measured $\mathrm{T}_{c}$ gradient, a process described in [18]. Films deposited in the SEGI, however, have demonstrated uniformities as good or better than typical postimplant samples from other systems. This "as-delivered" uniformity circumvents a full round of millikelvin testing ( $\mathrm{T}_{c}$ mapping) and ion implantation, increasing throughput rates. The consistency and uniformity of films produced by the SEGI may allow the test process to largely avoid $\mathrm{T}_{c}$ testing, aside from periodic verification. Circuit continuity tests can be accomplished at higher temperatures (up to $\sim 1 \mathrm{~K}$ ), meaning these detectors may be able to avoid dilution refrigerator testing as a whole during high throughput periods. With improved production throughput rates, the bottleneck is shifted from fabrication to testing, exaggerating the importance of these consistency and uniformity improvements.

\subsubsection{Conformal Film Deposition}

Controlling the fabrication quality of the aluminum-tungsten interface (to maximize quasi-particle diffusion into the TES) is important because phonons absorbed in the aluminum only contribute to the measured phonon signal if they are able to drift into the tungsten. When quasi-particles drift from the aluminum "fins" to the overlapping TES structure (see Figure 3.3), they must first drift into the intermediate 


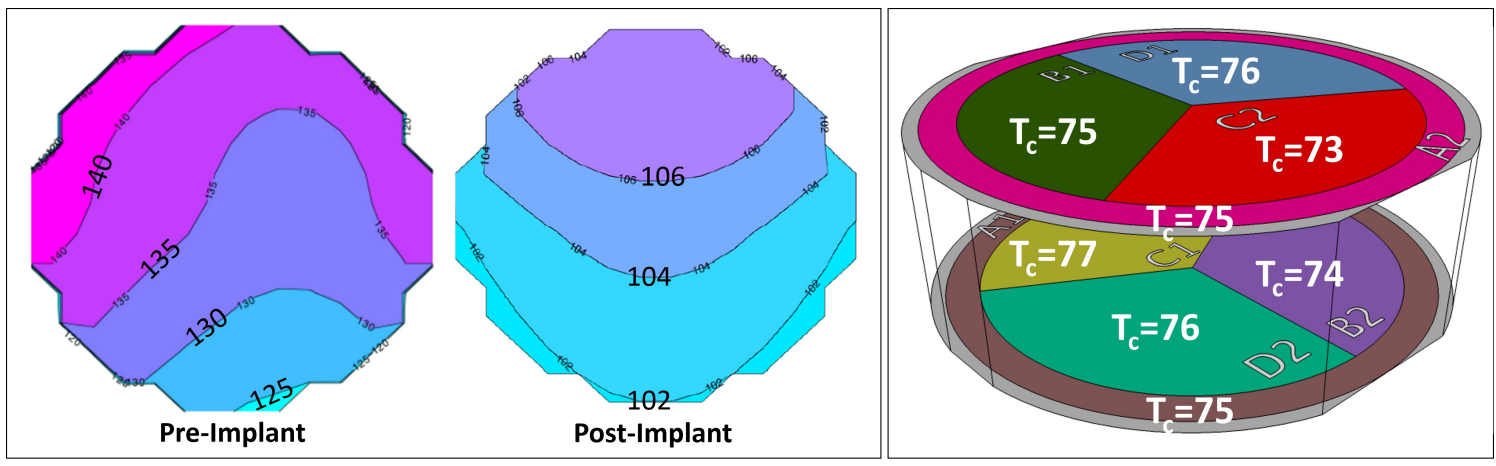

Figure 3.8: Left) Example of $\mathrm{W} \mathrm{T}_{c}$ variation (in $\mathrm{mK}$ ) across a sample substrate face from the previous CDMS deposition system. Middle) $\mathrm{T}_{c}$ variation of same film after ion implant compensation (see Section 3.2.2). Right) $\mathrm{T}_{c}$ measurements (see Table 3.2) from the 8 phonon channels of G9F, a detector fabricated at TAMU, demonstrating $\mathrm{T}_{c}$ uniformity without ion implantation. All $\mathrm{T}_{c}$ 's are in $\mathrm{mK}$.

tungsten cap layer. Since this intermediate tungsten layer is deposited immediately after the aluminum layer without breaking vacuum, no oxide is able to form between the two. Without this cap layer, an oxide forms on the aluminum surface before the TES layer deposition and inhibits the diffusion of quasi-particles from one film to the other. While the cap layer does oxidize slightly, the oxidation is easily removed with the RF etch which precedes the TES film deposition, forming a more favorable interface between the two tungsten layers.

The quasi-particle propagation from the "fins" to the TES's is aided by the bandgap disparity arising from the aluminum and tungsten films' contrasting $\mathrm{T}_{c}$ 's (aluminum's $\mathrm{T}_{c}$ of $\sim 1.2 \mathrm{~K}$ equates to a gap energy of $0.18 \mathrm{meV}$ compared to the tungsten's gap energy of $\sim 25 \mu \mathrm{eV})[13]$. Due to the magnitude of disparity in band gap energies, a process of quasi-particle multiplication can even occur at this boundary[19].

Because it is deposited over an already etched structure, the TES film must maintain continuity while stepping down $\sim 330 \mathrm{~nm}$ (the initial aluminum + tungsten layer) from the initial tungsten cap layer to the a-Si layer. Discontinuity in this 


\begin{tabular}{|c||c|c|}
\hline Channel & $\mathrm{T}_{c}(\mathrm{mK})$ & $\mathrm{R}_{n}(\Omega)$ \\
\hline $\mathrm{A} 1$ & 75 & 0.64 \\
$\mathrm{~B} 1$ & 75 & 0.59 \\
$\mathrm{C} 1$ & 77 & 0.59 \\
$\mathrm{D} 1$ & 76 & 0.59 \\
$\mathrm{~A} 2$ & 75 & 0.69 \\
$\mathrm{~B} 2$ & 74 & 0.56 \\
$\mathrm{C} 2$ & 73 & 0.54 \\
$\mathrm{D} 2$ & 76 & 0.56 \\
\hline
\end{tabular}

Table 3.2: Critical temperatures and "normal" resistance values $\left(\mathrm{R}_{n}\right)$ for the 8 phonon channels of detector G9F (see Section 3.2.2). $\mathrm{R}_{n}$ is the resistance of the channel while the aluminum is superconducting, but the tungsten is normal (held at a temperature significantly above its $\mathrm{T}_{c}$ ). Note: Channels A1 and A2 are outer channels (see Figure 3.8) and have higher $\mathrm{R}_{n}$ values due to their sensor layout.
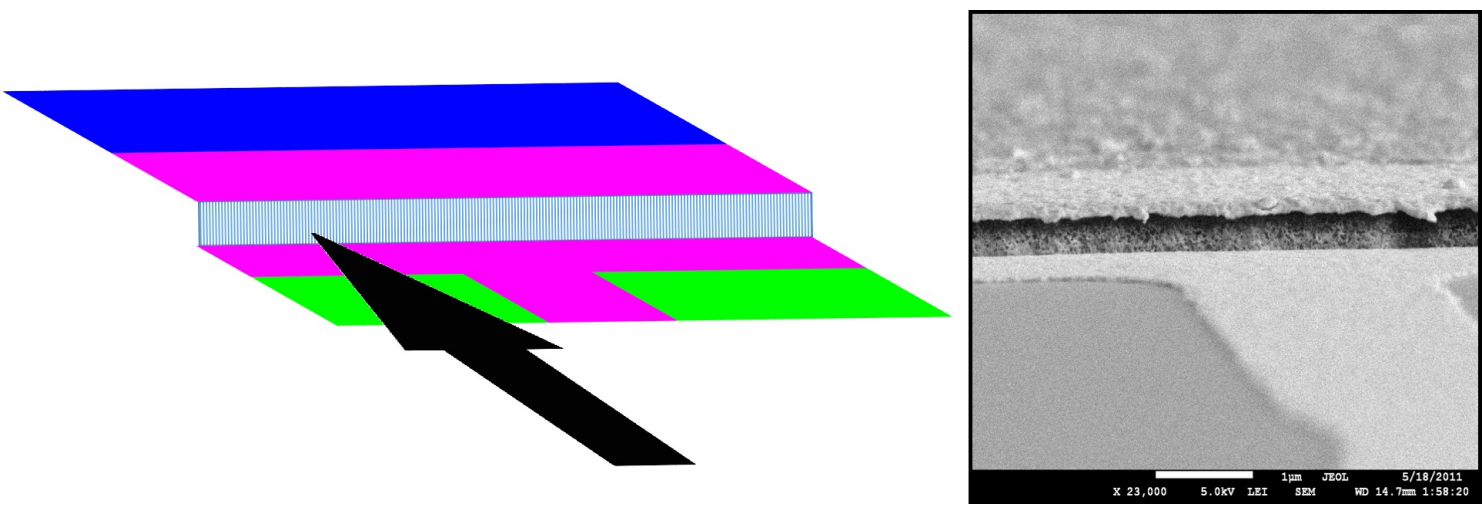

Figure 3.9: Close-up and SEM image of "waterfall" boundary (see Section 3.2.3). Location on phonon sensor and perspective are indicated by the arrow, referencing Figure 3.3.

region severs the phonon collection structure from the TES line (see Figures 3.3 and 3.9), preventing signal readout. To avoid this issue, the TES film must be a conformal layer closely following the topology, particularly the sidewall, of the trilayer structures. This region is designated as the "waterfall" region (see Figure 3.10 for examples of this feature exhibiting both poor and good continuity). To prevent this 


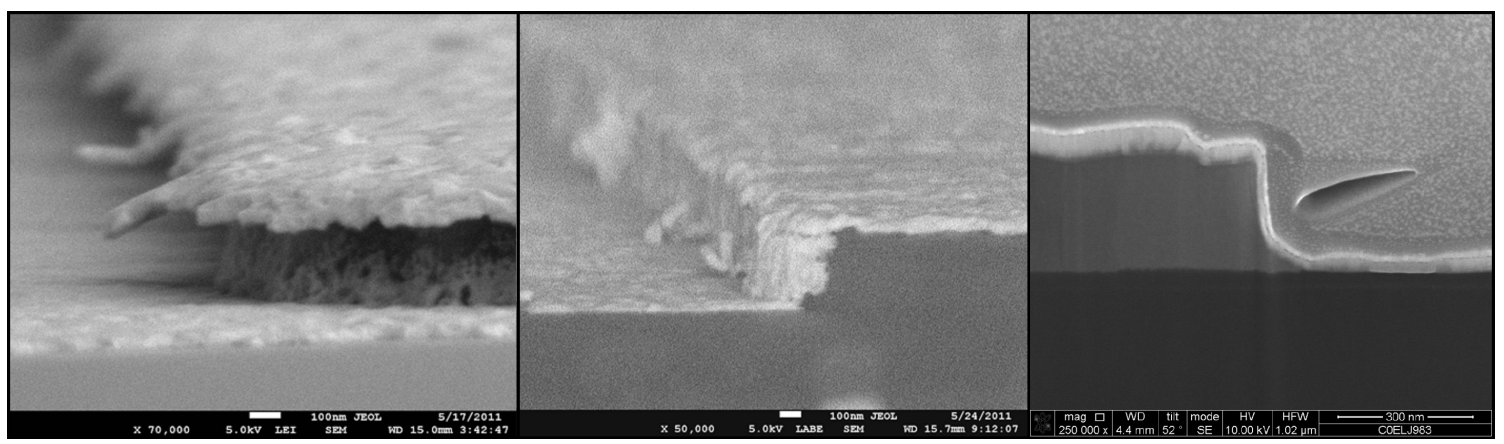

Figure 3.10: Left) SEM images of overhang discontinuity issue. Middle) Conformal sidewall deposition utilizing overhang etch, resulting in robust film continuity (see Section 3.2.3). Right) SEM image of FIB-prepared cross-section of waterfall feature, showing conformal tungsten deposition. FIB image courtesy of Evans Analytical Group.

problem, the "overhang etch" has been implemented into the process. This etch was tuned by performing many iterations of circuit fabrication on practice wafers with various overhang etch times using SEM imaging for feedback. Once established, the process was confirmed with thick substrates, again using SEM imaging.

\subsection{4 a-Si Etch and "Trenching" of iZIP Detectors}

Discrimination between background and signal events using these detectors relies on a calibrated ratio of energy measured in the ionization channels versus that measured in the phonon channels. Charge carriers produced by events near the faces of a detector often fail to drift through the entire crystal to the appropriate electrode. This results in a reduced ionization collection signal, causing the event to be improperly identified in subsequent analyses. A new circuit design has been implemented to combat this. The design is called the iZIP (interleaved Z-sensitive Ionization and Phonon detector)[22][23][24], and as the name suggests, it utilizes interleaved electrodes on each surface. The interleaved electrodes alternate from ground to $+2 \mathrm{~V}$ on one face and from ground to $-2 \mathrm{~V}$ on the other (see Figure 3.11). This is in contrast to 


\section{iZIP Charge Signal}

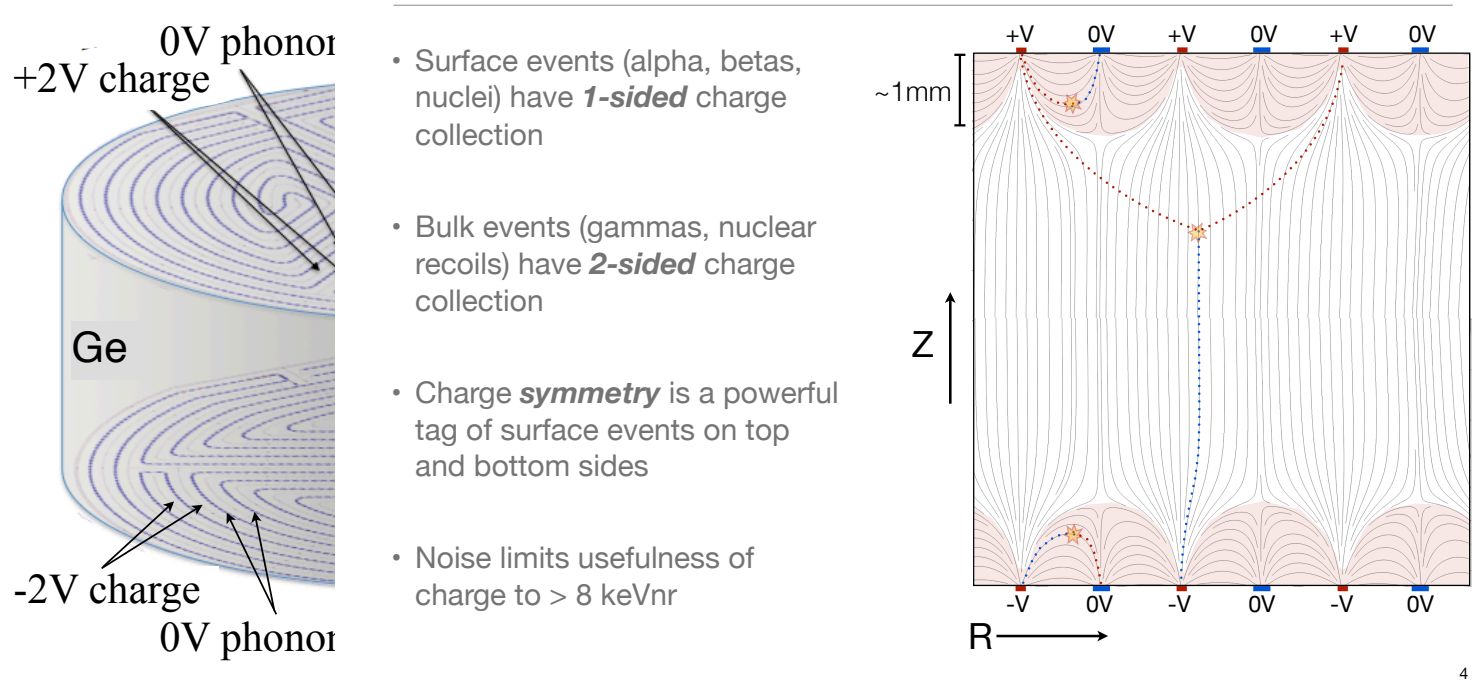

Figure 3.11: Left) Diagram of iZIP detector geometry and design used in SuperCDMS Soudan showing alternating biased charge collecting rails and $0 \mathrm{~V}$ phonon rails (see Section 3.2.4). Figure from [20]. Right) Simulation of iZIP internal field lines, showing strong tangential electric fields at the surface and a uniform drift field in the detector bulk, a method proven to discriminate against the previously problematic surface events. Figure from [21].

previous designs [25] where one face is held at ground potential while the other is voltage biased. The interleaved design produces a very uniform field in the bulk but local regions of high field intensity near the surface. This causes the carriers (electrons and holes) produced near the surface to both be collected by the adjacent surface, with relatively little charge drifting to the opposite face. Therefore, any events with significant disparities in charge collection from one face to the other (i.e. failing the charge-symmetry requirement) are considered to be surface events. This procedure has been demonstrated to be very successful and is the design currently operating in SuperCDMS Soudan[20]. To fully realize the potential of this technique, detectors should be able to hold higher biases (producing stronger local surface fields) without breakdown. Limitations arise, however, as the electrode spacing is $\sim 1 \mathrm{~mm}$, and current begins to leak across the surface as voltage is increased, eventually resulting in 
breakdown. To reduce this problem and allow higher bias voltage, a trench is etched into the surface of the substrate, between the electrodes. For this purpose, the a-Si etch step is extended by $\sim 700 \%$. Because the gas used to etch the a-Si also etches the substrate material, this extra time allows etching of the substrate itself. The process has been tuned such that a trench of $\sim 1 \mu \mathrm{m}$ in depth is created between the electrodes and has been shown[26] to produce detectors that can hold much higher bias voltages without the problems mentioned previously (see Section 4.4).

\subsubsection{Photoresist Studies}

The tuning of the photoresist layer is of utmost importance as it dictates not only the geometries of the final detector circuit structure, but whether or not the deposited films survive the fabrication process at all. For this reason, much time was spent investigating the photoresist layer and photolithographic processing of this layer. The cross-section of the developed photoresist pattern is controlled with the UV exposure, which can result in angled sidewalls (inward or outward), changing the width of the film etched below (see Figure 3.12). A dedicated study of the UV exposure (varying UV power and time, with SEM feedback) was performed to prevent these problems from affecting our circuit features.

In previous CDMS detector designs, Shipley Microposit S1813 photoresist[27] has been used for the photolithographic processing. This chemical was chosen for multiple reasons:

1. Resistance to etchant chemicals used in this process

2. Ability to reproduce sub-micron line-widths

3. Viscosity to match our desired layer thickness $(\sim 1.4 \mu \mathrm{m})$ with rotational speeds that produce optimum uniformity (3500-5500rpm [27])

4. Compatibility with metal ion-free developers [27] 


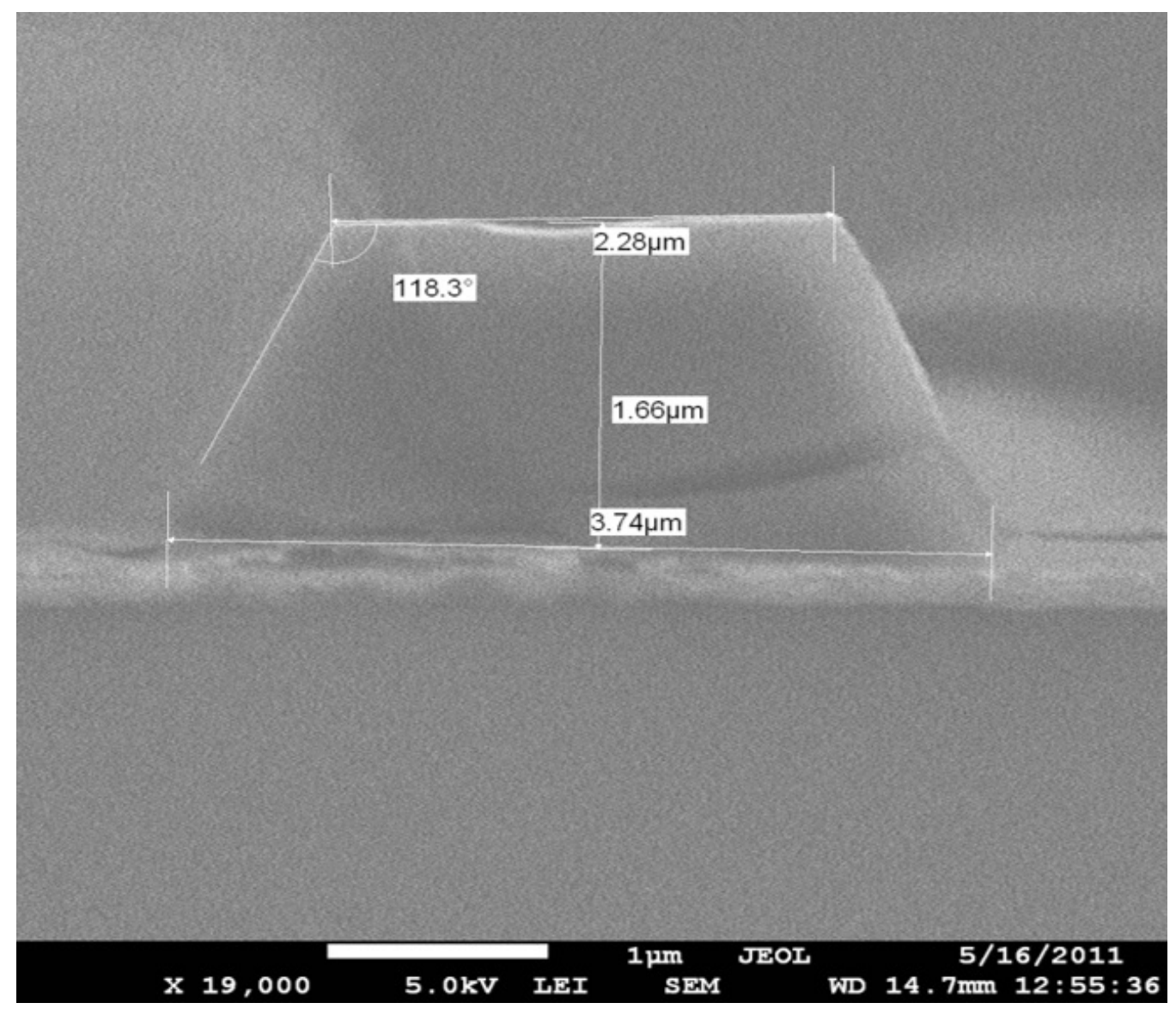

Figure 3.12: SEM image of a photoresist feature's cross-section. Due to improper UV exposure, this feature's sidewalls angle outwards, protecting a wider area of film from etching underneath, resulting in widening of circuit features. Note: This layer's thickness of $1.66 \mu \mathrm{m}$ corresponds to a previous spin coat recipe, using S1813 photoresist (see Section 3.2.5).

However, as substrates of larger mass joined the production line, it became desirable to decrease spin coating speeds (causing less strain on the spin coater and less risk to the substrates). For this reason, Shipley Microposit S1811[27] is now used for its lower viscosity while still possessing the other characteristics mentioned above. Using S1811, the spin coat process is performed at $2300 \mathrm{rpm}$ (as opposed to 4000 rpm required for S1813) for 60 seconds, producing a $1.4 \mu \mathrm{m}$ layer on each face. These 
parameters (along with those of the UV exposure mentioned previously) were tuned using feedback from SEM images confirming faithful reproduction of mask feature line-widths and robust cross-sections after exposure and development.

Another photoresist issue that was studied and successfully remedied is that of a so-called "edge bead". After the spin coat process, a thick bead of photoresist can be seen around the edge of the substrate surface. Thicker than the nominal layer, this bead does not receive enough UV exposure and developing to be properly patterned and removed prior to etching. While there are no vital circuit features in this region, it prevents any films underneath from being etched away, leaving a metal band (which could potentially cause shorts) around the edge of the detector after the photoresist is removed. It is for this reason that the previously mentioned shadow mask is implemented, preventing deposition in this region (see Section 3.1.6). This procedure has proven to be a low cost yet highly effective method of combating the edge bead problem, with negligible impact on detector patterning at radial extremities.

\subsection{Results to Date}

Using this process at the dedicated TAMU fabrication facility, detectors have been produced of the size and design of those in SuperCDMS Soudan. Test data from detector G9F, one of the first of these produced at TAMU, can be seen in Figure 3.13, demonstrating pulses from operational phonon sensor channels as well as the 356 keV photopeak from a Ba-133 calibration source. In addition, this detector showed unparalleled TES $\mathrm{T}_{c}$ uniformity without ion implant compensation (see Figure 3.8 and Table 3.2). However, it showed an inability to hold adequate bias voltage, leading to further tuning of the trenching process (see Section 3.2.4). The following detector, G10F (using the improved trenching process), demonstrated more than adequate ability to hold bias, showing no signs of leakage up to $\pm 5 \mathrm{~V}$ (the limit of the test 
stand). Specification standards used to rate SuperCDMS detectors categorize this detector as "very good". Subsequent testing showed functional charge performance up to $9 \mathrm{~V}$ [26], much higher than required for the experiment.

Detectors produced at this facility have demonstrated performance that meets or exceeds the requirements for this experiment, certifying this location as an integral fabrication facility for SuperCDMS SNOLAB detectors. $100 \mathrm{~mm}$ x $33.3 \mathrm{~mm}$ thick science quality detectors were successfully produced at this facility in early 2013 (see Figure 3.14).
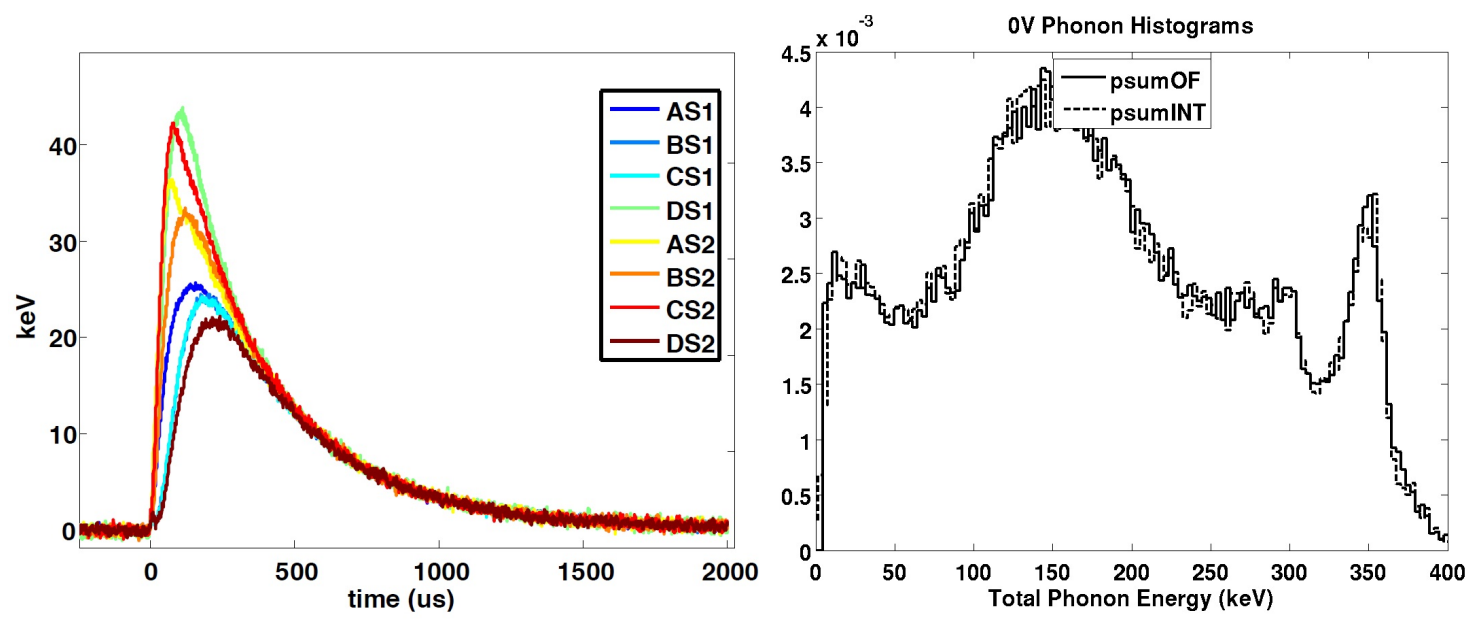

Figure 3.13: Left) Phonon pulses from detector G9F, fabricated at TAMU. Right) Calibration spectrum from detector G9F, clearly showing the $356 \mathrm{keV} \mathrm{Ba}^{133}$ peak (see Section 4.4). 


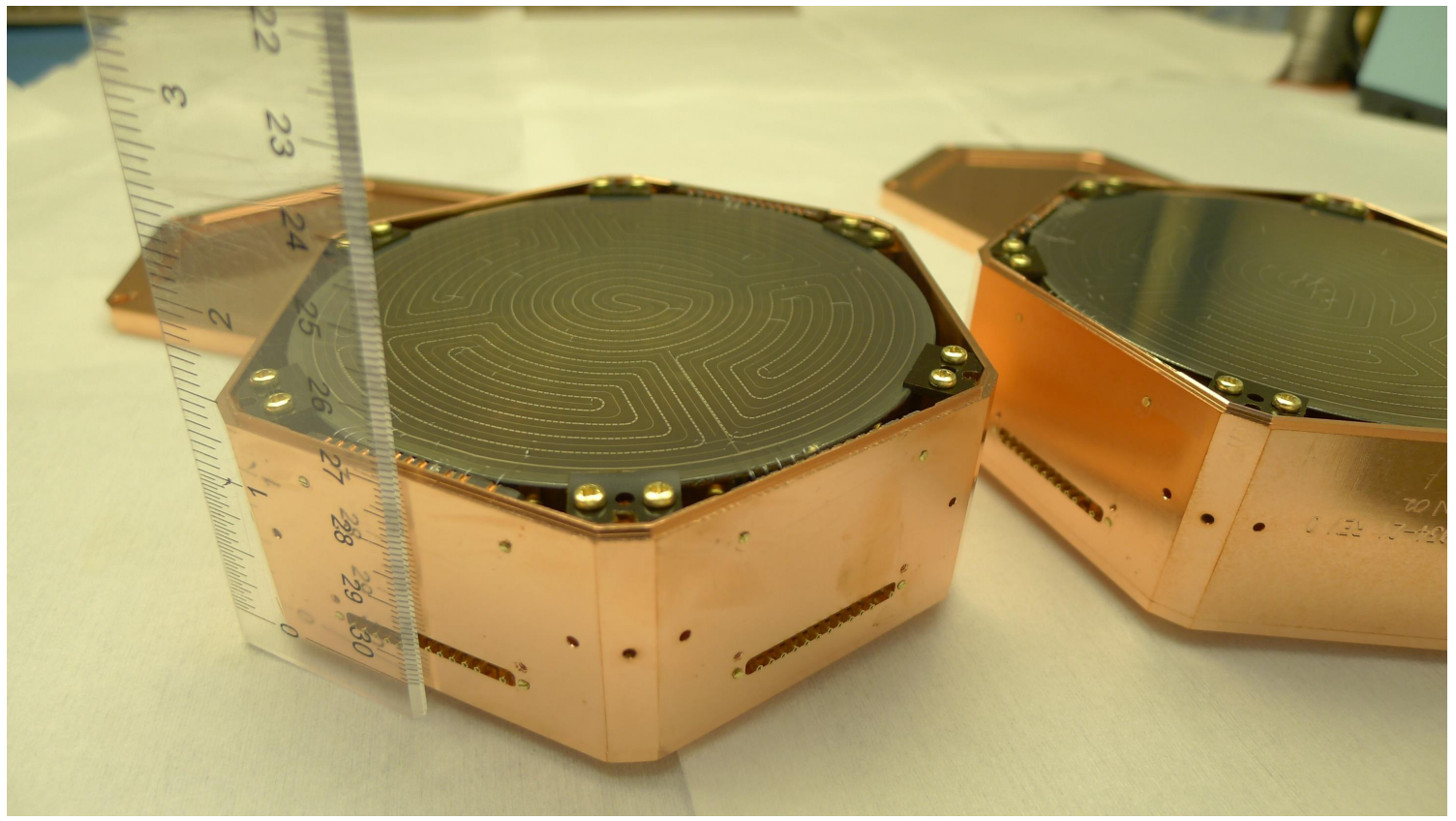

Figure 3.14: $100 \mathrm{~mm} \times 33.3 \mathrm{~mm}$ detectors fabricated at TAMU. 


\section{LOW ENERGY NUCLEAR RECOIL MEASUREMENTS IN GERMANIUM}

\subsection{Introduction}

Understanding low energy nuclear recoils in various elements has become an increasingly important topic recently, due to the latest results from direct detection dark matter experiments. The nuclear recoil signal is that which is expected from a WIMP interaction, and recent results from these experiments (CDMSII silicon[28], for instance) have shown possible hints of WIMP signals in the light mass (low energy deposition) parameter space. Current analyses of these experiments rely on predictions of expected detector response to low energy nuclear recoils based on theoretical treatments of energy dissipation and stopping powers explored and published by Lindhard et al. in 1961 and 1963 ([29], [30], [31]). The relationship in question is the ratio (termed the 'Lindhard factor', see Figure 4.1, and Section 2.3.1) of ionization energy produced by a nuclear recoil vs that of an electronic recoil (the typical calibration scale for these detectors). Since then, experiments have measured and largely verified the predicted Lindhard factor in various elements, but the uncertainties in the low energy measurements still need to be improved to make confident claims of WIMP signals in this region. In this work, an infrastructure is designed and used to support such measurements in germanium. This is accomplished by scattering neutrons of known energies off of a germanium detector at specific angles, producing nuclear recoils of specific energies.

\subsection{Experimental Setup}

Low energy nuclear recoils of known energies are typically difficult to produce for calibration purposes due to the energy spectra of common radioactive neutron sources. While these spectra produce features that can be used for calibration, they 


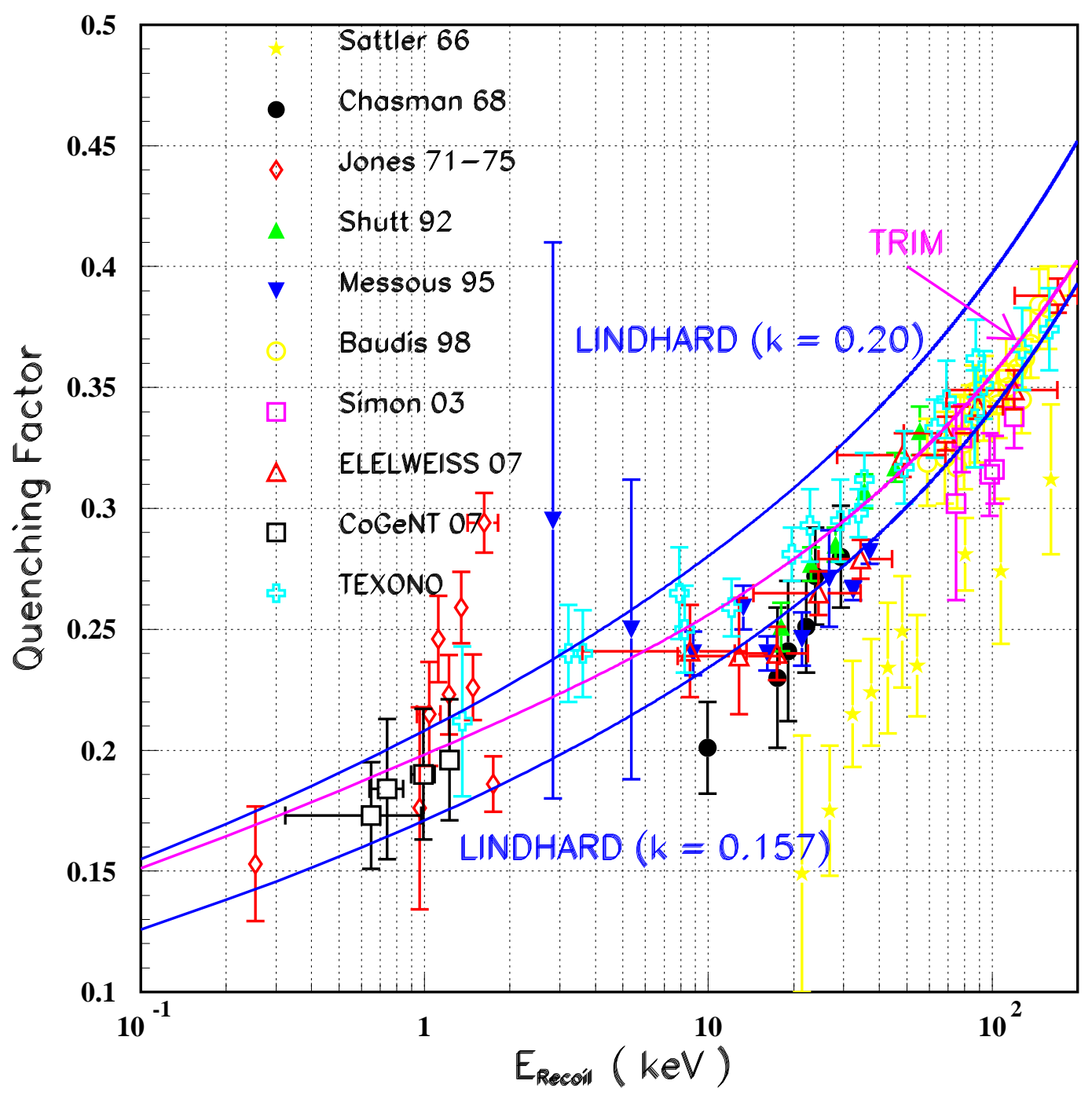

Figure 4.1: Current measurements of the Lindhard factor in germanium [32]. 
are typically at much higher energies than those of interest here. It is possible to produce lower energy neutrons using radioactive sources to excite beryllium. Two elements commonly used for this purpose are yttrium and antimony (specifically, ${ }^{88} \mathrm{Y}$ and ${ }^{124} \mathrm{Sb}$ ). These sources produce neutron energies of 166 and $26 \mathrm{keV}$, respectively [33]. This method is currently being used by other members of the CDMS collaboration to make similar measurements, with results and publication expected in the near future.

Ideally, one would use a mono-energetic neutron beam of tunable energy and intensity. This experiment uses tools to replicate those parameters to the best of current abilities in the field. This is accomplished using a proton beam (see Appendix B) of stable, tunable energy incident upon a LiF target, producing a subsequently stable, tunable pseudo-mono-energetic neutron beam (see Section 4.2.1).

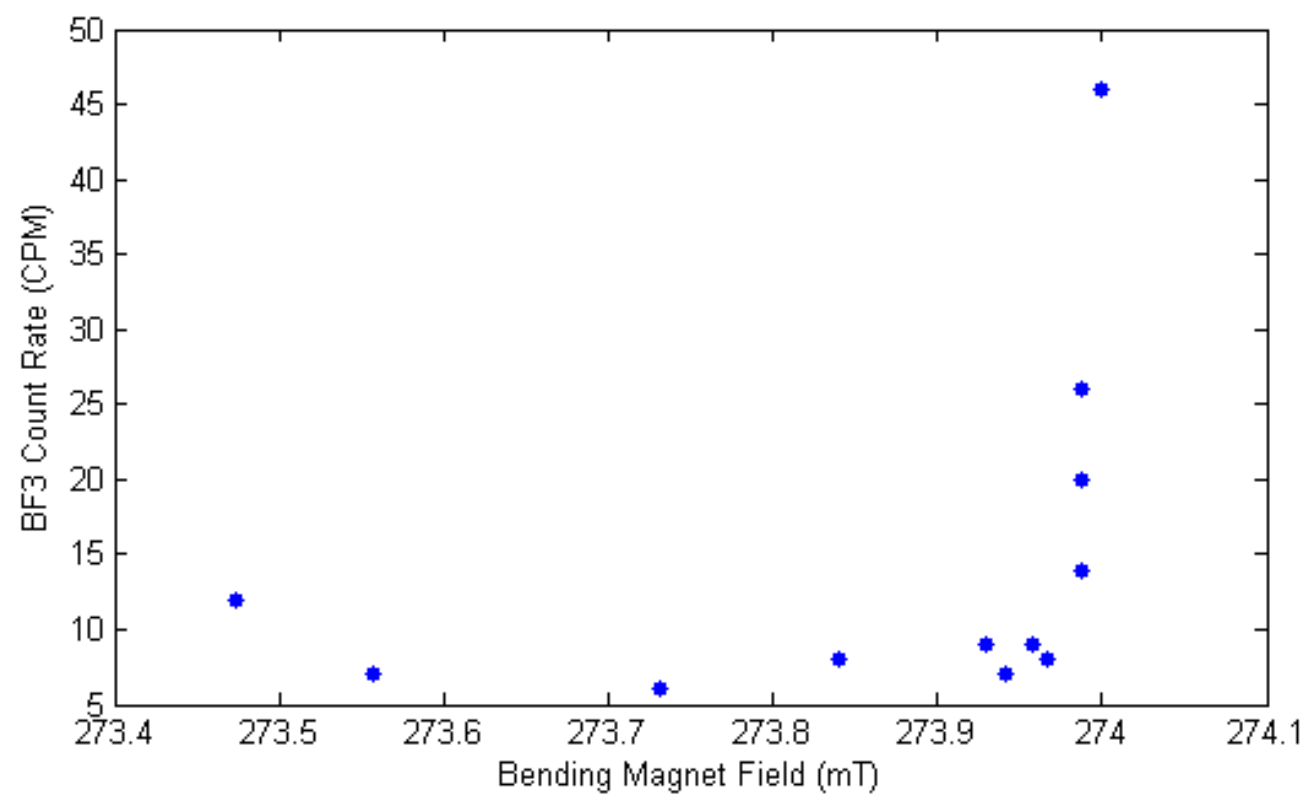

Figure 4.2: Neutron rate (measured with a $\mathrm{BF}_{3}$ counter enclosed in polyethylene case) vs. bending magnetic field (a measure of incident proton energy). The threshold in this case is measured within $21 \mu \mathrm{T}$. 


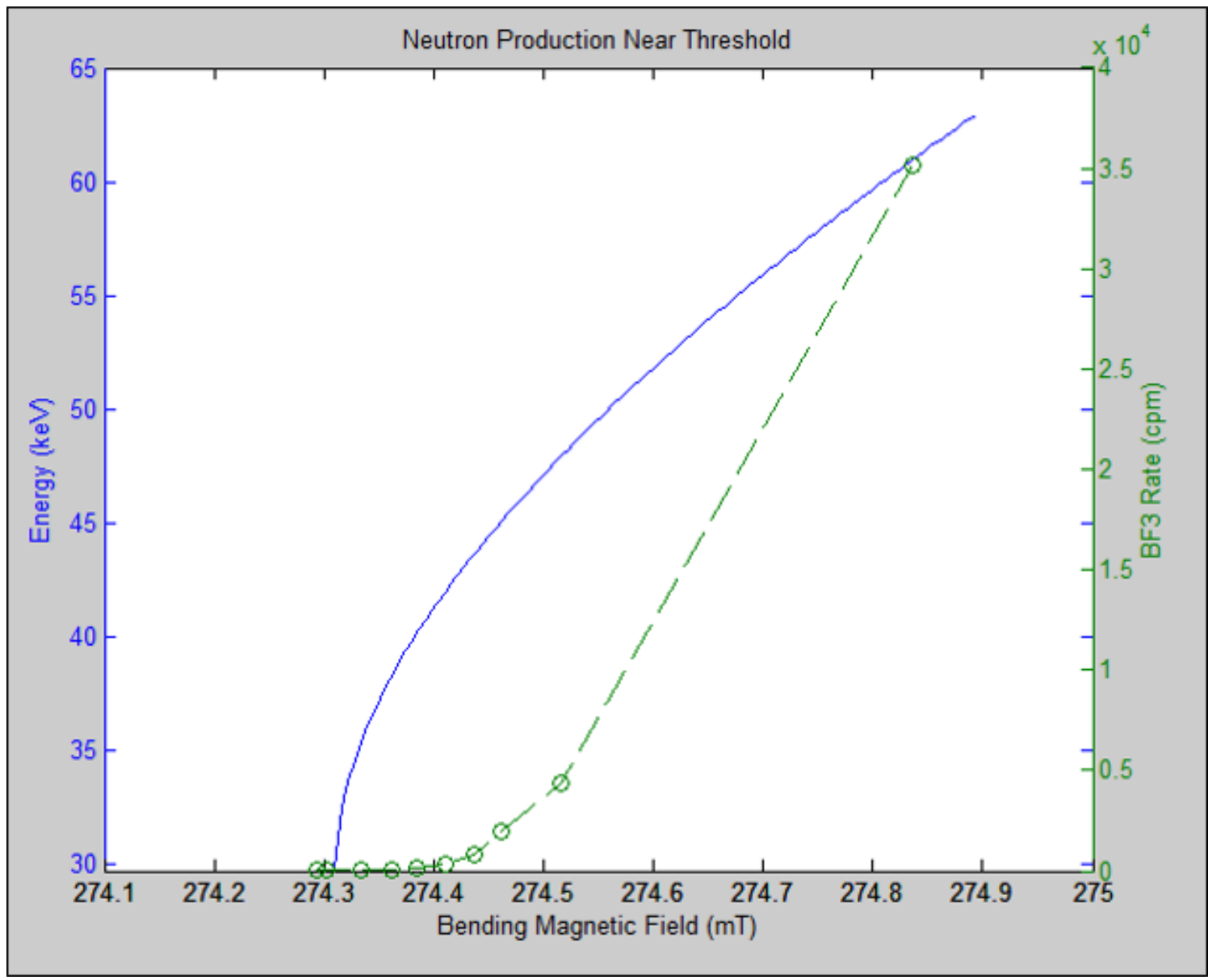

Figure 4.3: Neutron energy (solid line) and neutron detection rate (dashed line) vs. bending magnetic field. Neutron rate is measured with a $\mathrm{BF}_{3}$ counter enclosed in polyethylene.

\subsubsection{Proton Beam and Neutron Production}

The collision of protons above a certain threshold energy upon lithium atoms induces a ${ }^{7} \mathrm{Li}(\mathrm{p}, \mathrm{n}){ }^{7}$ Be reaction. This threshold energy $(1.88 \mathrm{MeV}$ [34], producing 29.7 $\mathrm{keV}$ neutrons) is used to calibrate the proton beam energy before each run. Starting below threshold, the proton beam energy is increased while observing the neutron detection rate in $\mathrm{BF}_{3}$ counter (see Figures $4.2 \& 4.3$ ) placed at the beam end allows one to precisely calibrate the beam energy. In order to increase the proton 


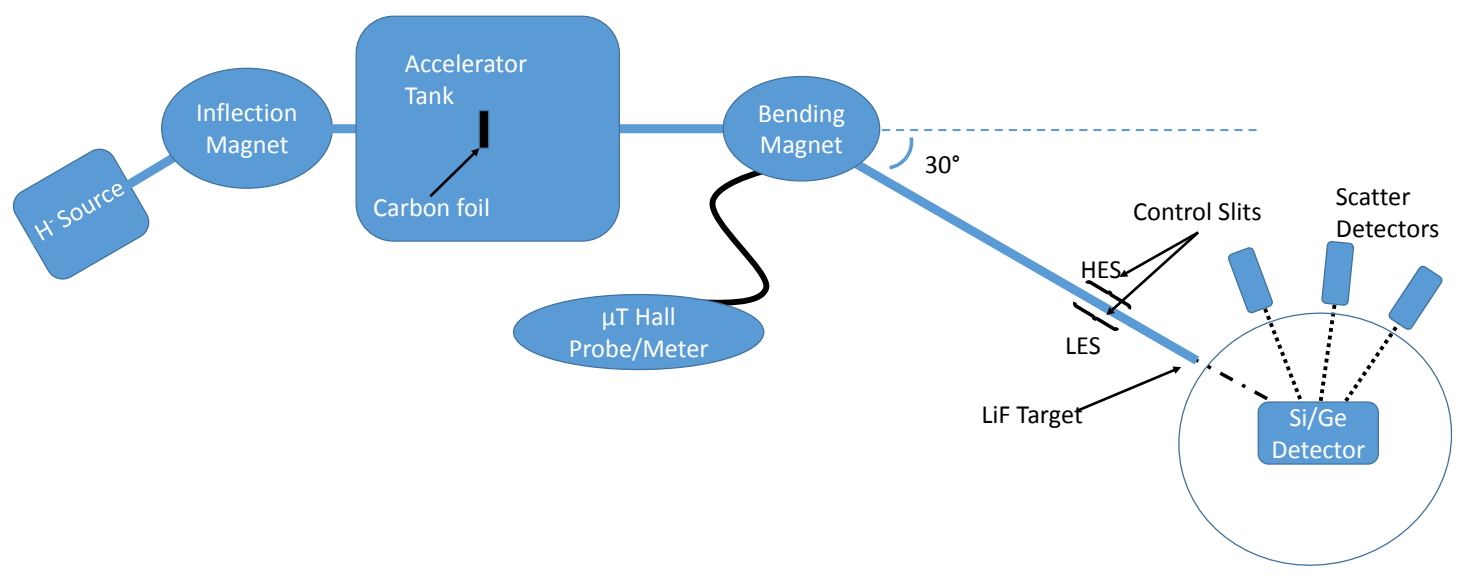

Figure 4.4: Diagram of the proton beam setup, neglecting steering, focusing, and measurement components (see Section 4.2.1). Not to scale.

beam energy, the bending magnet (see Figure 4.4) is adjusted and measured precisely, while the accelerating potential compensates accordingly using feedback from sensors downstream. In this way, the bending magnet is the only adjustment used to tune the proton beam energy. This magnet is incredibly stable, and its field is precisely measured to $\pm 1 \mu \mathrm{T}$ using a high-quality temperature-compensated Hall probe read out by a Gauss/Teslameter (F.W. Bell Model 8010). Using the known threshold proton energy and its corresponding bending magnet field, one can find the bending field required for any other proton energy desired using the following equation:

$$
E_{p}=\frac{q^{2} B^{2}\left(a^{2}+d^{2}\right)^{2}}{8 m_{p} d^{2}}
$$

In this equation, the proton energy $\left(\mathrm{E}_{P}\right)$ and bending field $(\mathrm{B})$ are the only variables. The constants q (proton charge), a \& d (magnet dimensions), and $\mathrm{m}_{p}$ (proton mass) can thus be combined into a single constant, $\mathrm{k}$, simplifying the equation to 
the following:

$$
E_{p}=k B^{2}
$$

The constant $\mathrm{k}$ is found using the proton energy at the neutron production threshold with a known B-field. This allows calculation of the proton beam energy from the bending field value alone. With a given incident proton energy, one can calculate the outgoing neutron spectrum, using the following equation from [33]:

$$
\begin{array}{r}
E_{n}=E \frac{m_{G} m_{n}}{\left(m_{n}+m_{r}\right)^{2}}\left\{2 \cos ^{2} \theta+\frac{m_{r}\left(m_{r}+m_{n}\right)}{m_{G} m_{n}}\left[\frac{Q}{E}+\left(1-\frac{m_{G}}{m_{r}}\right)\right]\right. \\
\left. \pm 2 \cos \theta \sqrt{\cos ^{2} \theta+\frac{m_{r}\left(m_{r}+m_{n}\right)}{m_{G} m_{n}}\left[\frac{Q}{E}+\left(1-\frac{m_{G}}{m_{r}}\right)\right]}\right\}
\end{array}
$$

In this equation, $\mathrm{E}_{n}$ is the outgoing neutron energy, $\mathrm{E}$ is the incoming proton energy, $\mathrm{m}_{G}, \mathrm{~m}_{n}$, and $\mathrm{m}_{r}$ are the masses of the projectile nucleus (proton), neutron, and residual nucleus $(\mathrm{Be})$, respectively, $\theta$ is the neutron emission angle, and $\mathrm{Q}$ is the $\mathrm{Q}$ value for the ${ }^{7} \operatorname{Li}(\mathrm{p}, \mathrm{n})^{7} \mathrm{Be}$ reaction. Note that the angular dependence prevents this from being a truly mono-energetic neutron beam. However, given a small solid angle subtended by a target (at which neutrons are projected), the spread in neutron energies can be made quite small (see Figures $4.5 \& 4.6$ ).

It should also be noted that, below a certain proton energy $(\sim 1.92 \mathrm{MeV})$, the neutron energy is double valued. This is due to the fact that in the center of mass frame, there is a forward traveling population and a backward traveling population. The neutron energies used in this work are much higher, and thus, single valued (see Figure 4.6 for example). This equation (4.3) also tells us the spread in neutron energy due to the spread in incoming proton energy. 


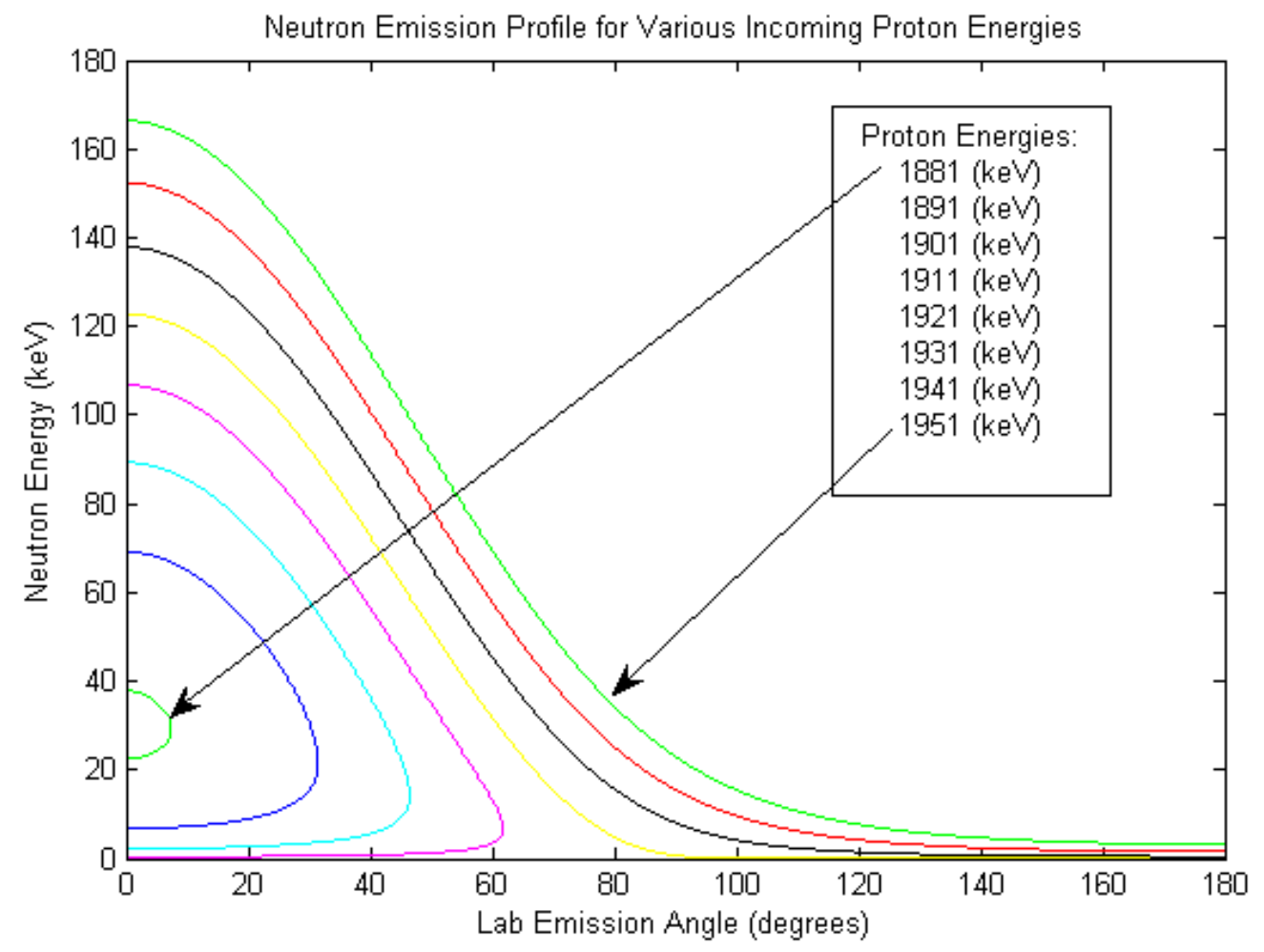

Figure 4.5: Plot of neutron energies at various angles for given proton energies (from Equation 4.3). 


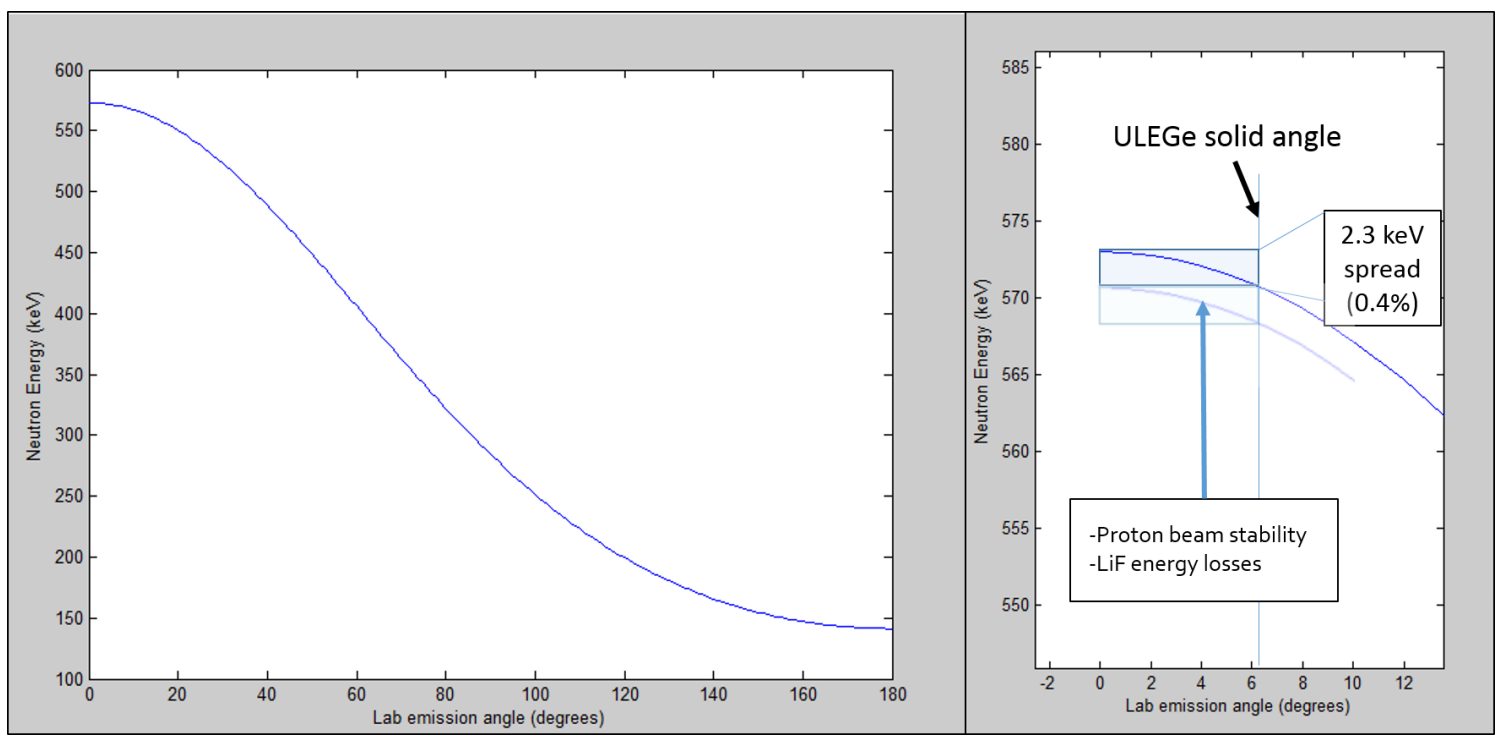

Figure 4.6: Left) Example neutron spectra of the approximate energy used in this work. Note, the angular dependence is minimal at $0^{\circ}$ (used in this experiment), and $180^{\circ}$. Right) Demonstration of sources of uncertainty in incident neutron energy. The $\mathrm{x}$-axis spread in energy is due to the solid angle of the detector and the angular dependence of neutron production, resulting in a $0.4 \%$ uncertainty based on the target detector and configuration used in this work. The vertical shift is due to uncertainties in the proton energy at the point of the ${ }^{7} \mathrm{Li}(\mathrm{p}, \mathrm{n})^{7}$ Be reaction (see Section $4.3)$. 


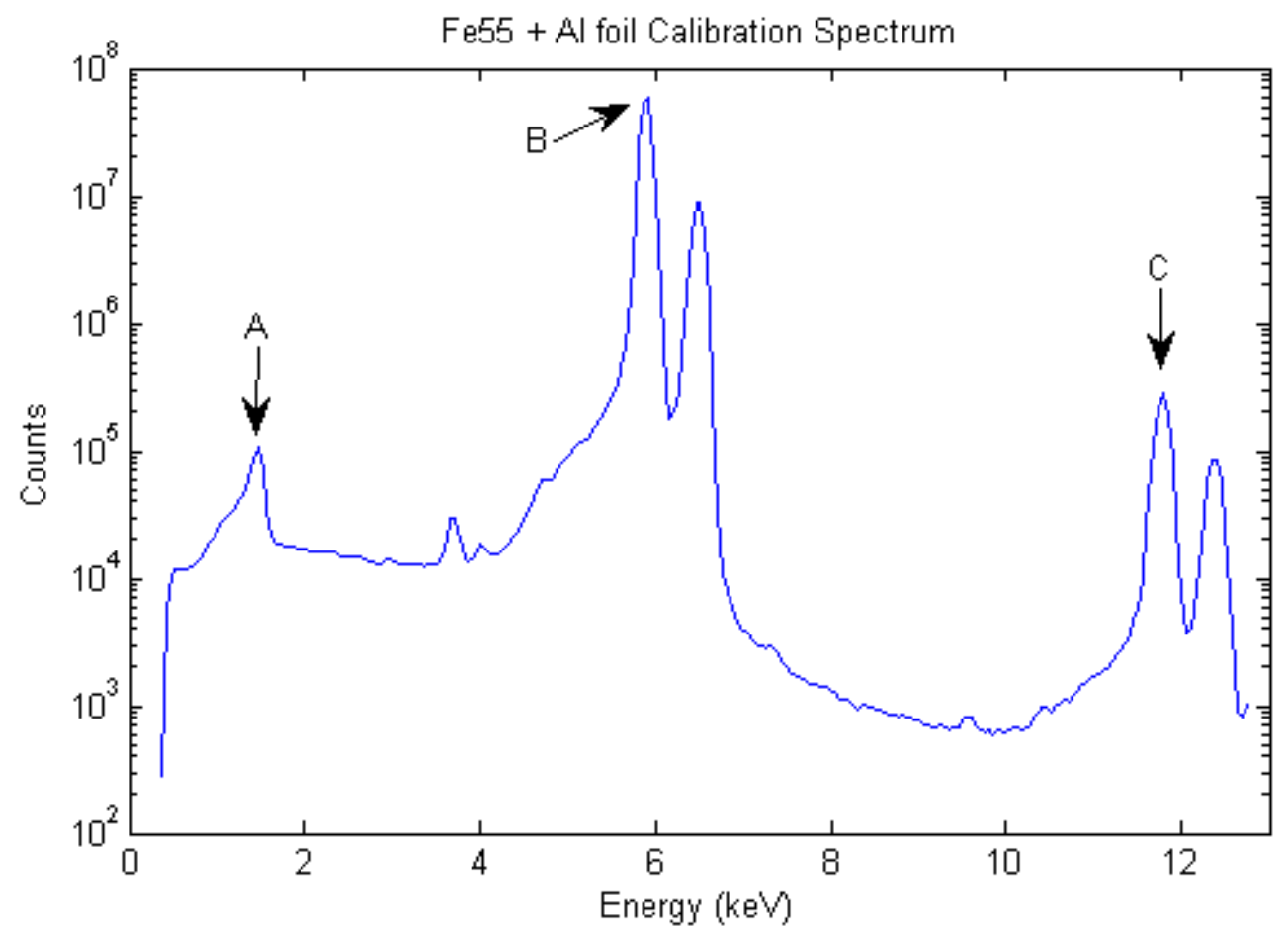

Figure 4.7: Example calibration spectrum obtained with the Ge detector. This spectrum is the result of an ${ }^{55} \mathrm{Fe}$ source on a sheet of aluminum foil on the detector window. Three peaks are used for calibration: A) Al $\mathrm{K} \alpha$ (from the foil) $=1.48$ $\mathrm{keV}, \mathrm{B}) \mathrm{Mn} \mathrm{K} \alpha$ (from the ${ }^{55} \mathrm{Fe}$ ) $=5.89 \mathrm{keV}$, and C) Pile-up of two simultaneous Mn $\mathrm{K} \alpha$ events $=11.78 \mathrm{keV}$. These three peaks provide a good measure of linearity and resolution across the full scale of the MCA. 


\subsubsection{Detector Setup}

The detector being characterized in this experiment is an Ultra-LEGe (Ultra-Low Energy germanium) commercially available from Canberra Industries. The three scatter-tagging detectors are PMT instrumented scintillators; two are NaI and the other is NE213. The liquid scintillator NE213 is a natural choice (high hydrogen content yields higher energy deposition from incoming neutrons, and NE213 has the capability of $\mathrm{n} / \gamma$ pulse-shape discrimination. While not as efficient at neutron tagging, the $\mathrm{NaI}$ detectors were also included as they proved sufficiently effective early on, given appropriate external event discrimination (see Section 4.2.3).

The Ultra-LEGe detector (or more precisely, it's pre-amp) is directly read out by a LYNX ${ }^{\circledR}$ DSP/MCA module. This unit provides precise energy measurements as well as various I/O options to control the data acquisition process (see Section 4.2.4). The Ge detector is calibrated before every run using an ${ }^{55} \mathrm{Fe}$ source in conjunction with Al foil (see Figure 4.7). These calibrations have shown excellent linearity and stability in this detector.

The scintillators were roughly calibrated at the start of the experiment using ${ }^{241} \mathrm{Am}(60 \mathrm{keV} \gamma)$ and ${ }^{22} \mathrm{Na}(511 \mathrm{keV}$ annihilation $\gamma$ and $1275 \mathrm{keV} \gamma)$ to give an idea of what pulse heights to expect from incident neutrons. This was later verified in situ (see Section 4.2.3). Precise calibration is not necessary however, as the energy measured by these detectors does not need to be well defined to provide effective neutron tagging.

Careful shielding, however, is required for successful tagging of neutron scatters. In this work, $\mathrm{Pb}$ is used both on the beam-end (to block gammas produced in the proton collisions) and around the scatter detectors (to prevent false coincidence events

due to ambient and beam gammas (see Figure 4.8). In addition, wedges of polyethy- 


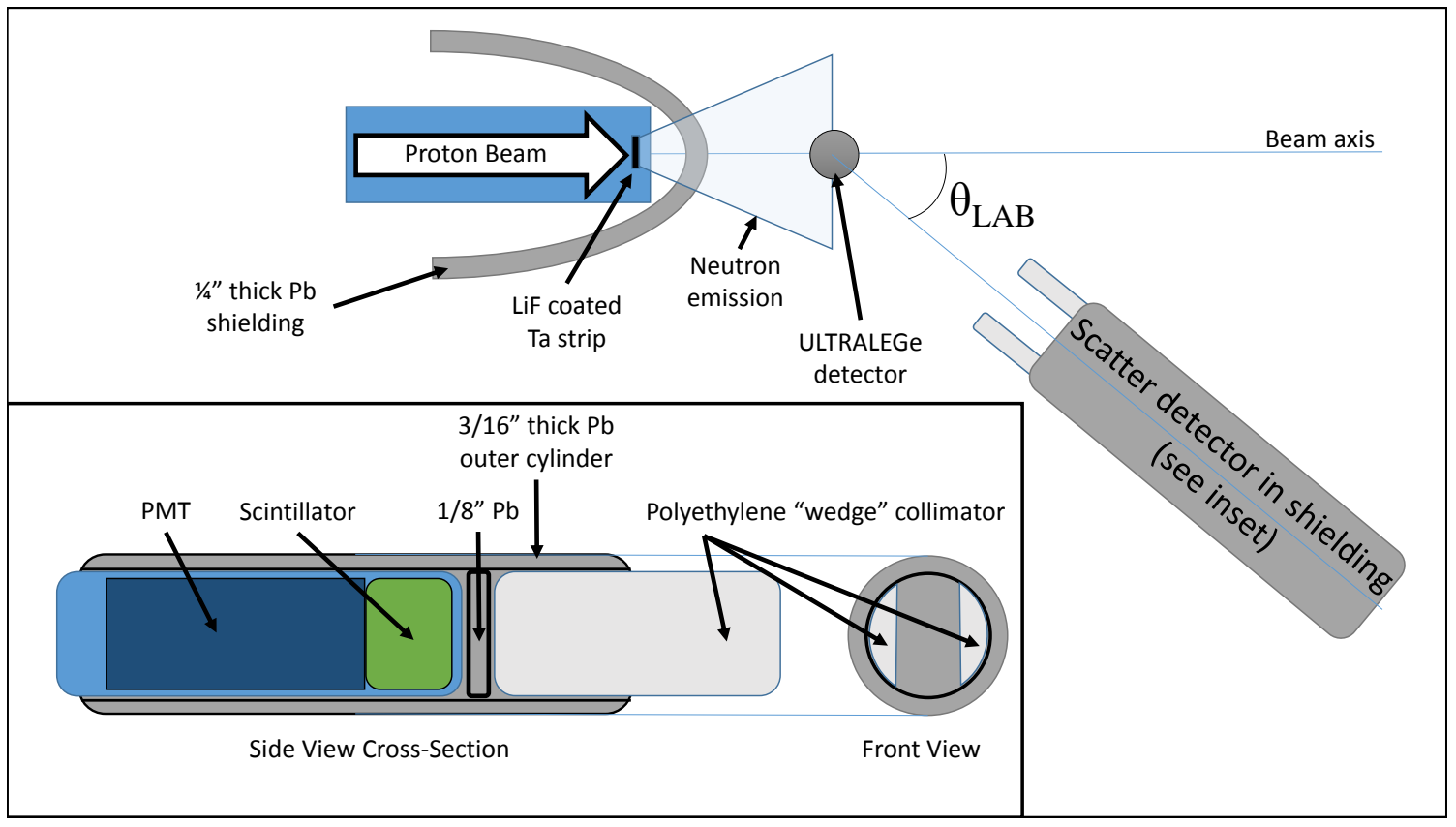

Figure 4.8: Diagram showing the end-beam setup. Inset) Diagram of scatter detector shielding and collimation.

lene are used to narrow the acceptance window of neutrons, defining a smaller solid angle in energy $(\theta)$ space while maintaining the full detector diameter solid angle in the perpendicular direction. In this way, the peak produced in the Ge spectrum is better defined without loss of statistics at the peak (see Figure 4.9 and Section $4.2 .6)$.

\subsubsection{Signal vs. Background Event Discrimination}

For a successful measurement, careful discrimination must be implemented to reject background events and retain the events from the desired scattering process. Two handles are used in this process: the energy measured in the scatter detector, and the timing between the Ge event and the subsequent scatter detector event.

Using Monte Carlo simulations as well as experimental data, an energy window (using a lower- and upper-level discriminator, LLD and ULD) is chosen such that 


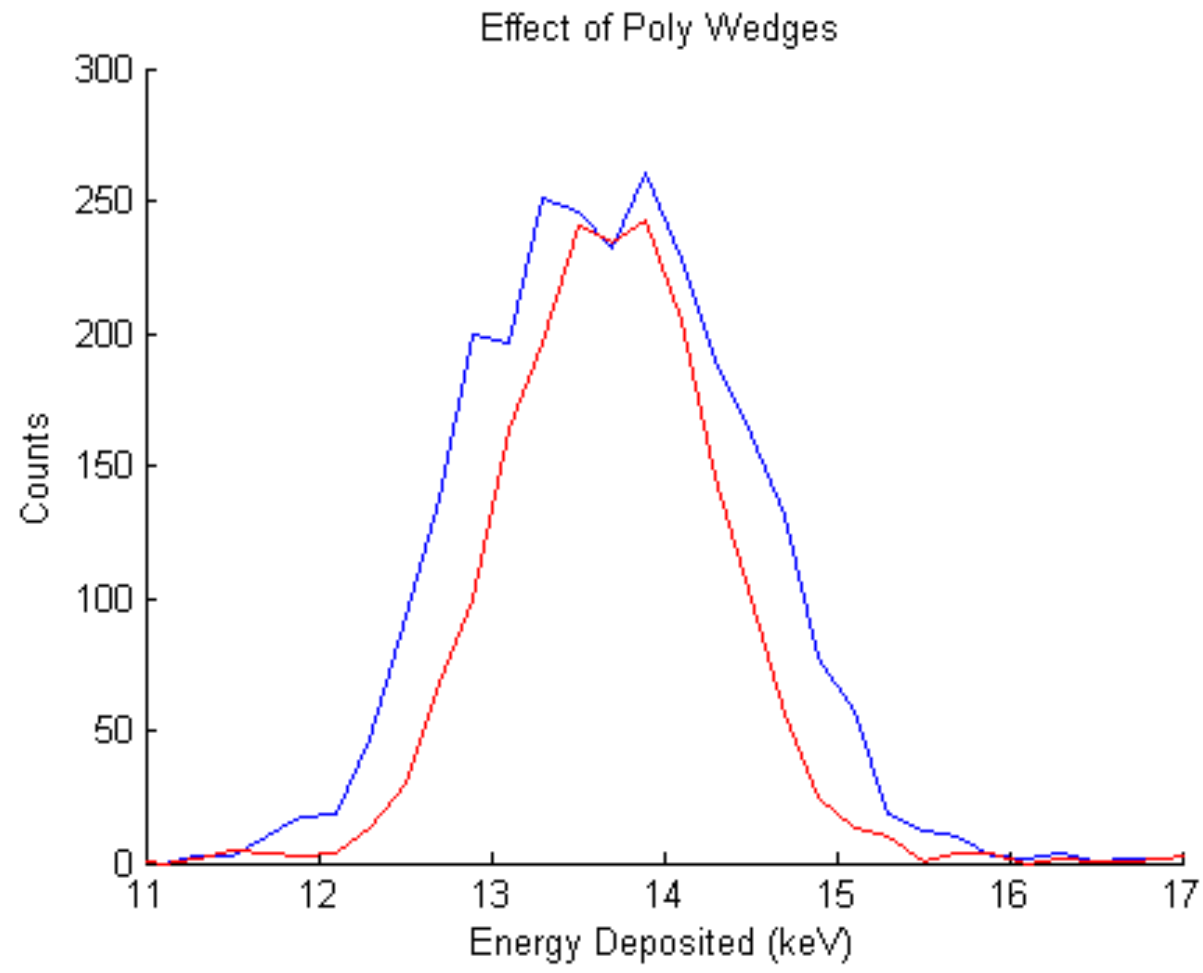

Figure 4.9: Effect of scatter detector collimator, as simulated in GEANT, showing the peak narrowing due to the polyethylene without loss of peak statistics. 

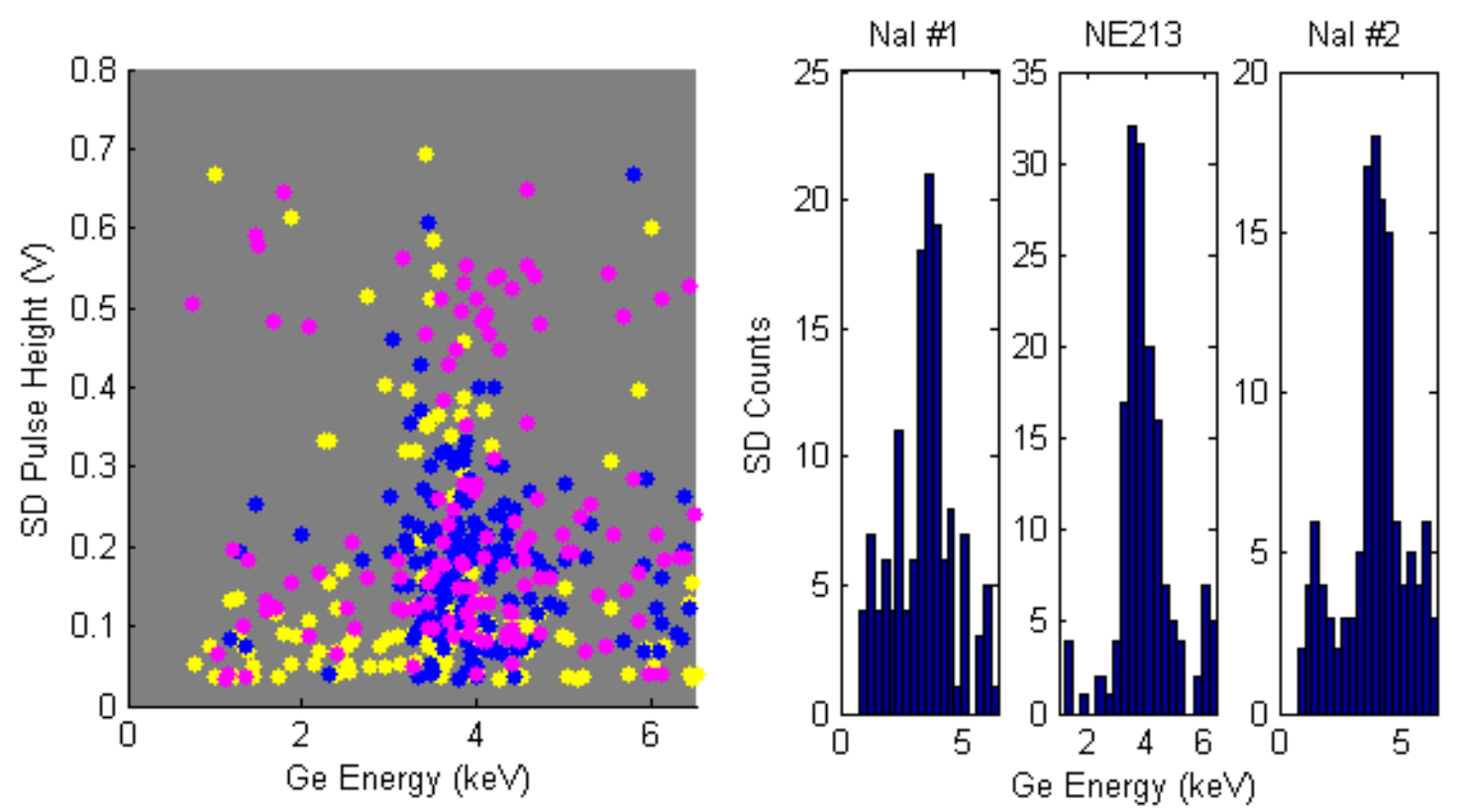

Figure 4.10: Left) Scatter detector data showing which pulse heights correspond to the ROI in ionization energy measured in the Ge detector. This population determines hardware settings for upper- and lower-level discriminators for accepting scatter detector pulses. Right) Histograms showing the ROI in each detector under these discriminator settings. Neutron scatter peak is clearly visible in all three. See Section 4.2 .3

a sufficient neutron event acceptance is ensured while excluding background events. Experimental data is logged using a 4 channel oscilloscope triggered on accepted events (SCA output). Upon triggering, pulse heights of each scatter detector are measured as well as the MCA's auxiliary 'analog out' signal. This signal is proportional to the Ge ionization energy. Plotting these shows a population of scatter detector pulse heights corresponding to desired neutron scatter events (see Figure 4.10). To maintain effective discrimination at various neutron beam energies, the LLD and ULD are scaled accordingly for each run.

Without precise timing information, the scatter events in the final Ge spectrum would be entirely buried by background events (false coincidences). Therefore, this 


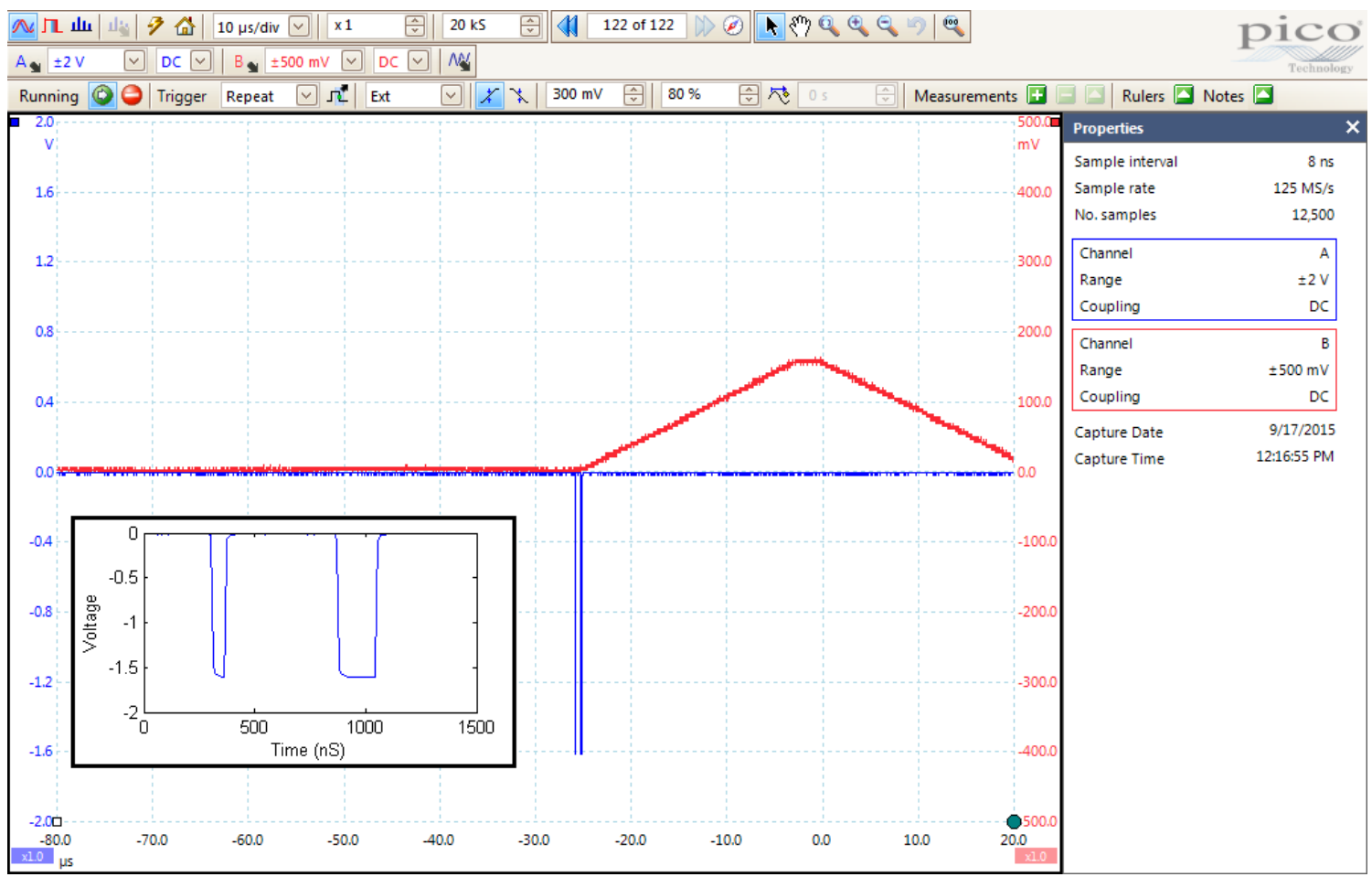

Figure 4.11: Display of 2 channel scope used to monitor and analyze timing information. The red channel is the 'analog' Ge ionization energy channel (an auxiliary output of the MCA) which has an amplitude proportional to the ionization energy. The blue channel measures the two combined timing pulses: the ICR pulse from the MCA (signaling the detection of a Ge ionization event) and the scatter detectors' combined discriminator output, signaling a scatter detector ionization event. Inset) Zoomed image of a scatter detector pulse followed by an ICR pulse. This gap is used to define the timing settings in the timing and logic hardware (see Section 4.2.3).

aspect is carefully examined and tuned to maintain appropriate discrimination. Due to the time for charge readout and electronics processing, the ICR signal (incoming count rate, a signal which is triggered in the DSP's fast channel to signal the initial detection of a possible Ge event) actually triggers after the subsequent scatter detector discriminator trigger (even though the Ge event occurs first in real time). For reliable discrimination, this delay needs to be well defined and tuned in the logic and timing hardware. A PC oscilloscope is used as a DAQ to analyze the delays for 
proper neutron scatter events, recording the timing pulses as well as associated Ge ionization energy (see Figure 4.11). The timing pulse delays and corresponding Ge energy measurements are then plotted to find and confirm the appropriate timing for scatter events (see Figure 4.12). For the neutron energies and distances used in this experiment, time of flight variations are not large enough to require tuning timing parameters on a run-by-run basis. However, due to other reasons, it turns out that the timing parameter does need tuning corrections for lower energy depositions in the Ge detector (see Section 4.2.5).

With these two windows (timing and energy) tuned, the scatter detectors reliably tag neutron events with acceptable background event rates.

\subsubsection{Logic and Timing Setup}
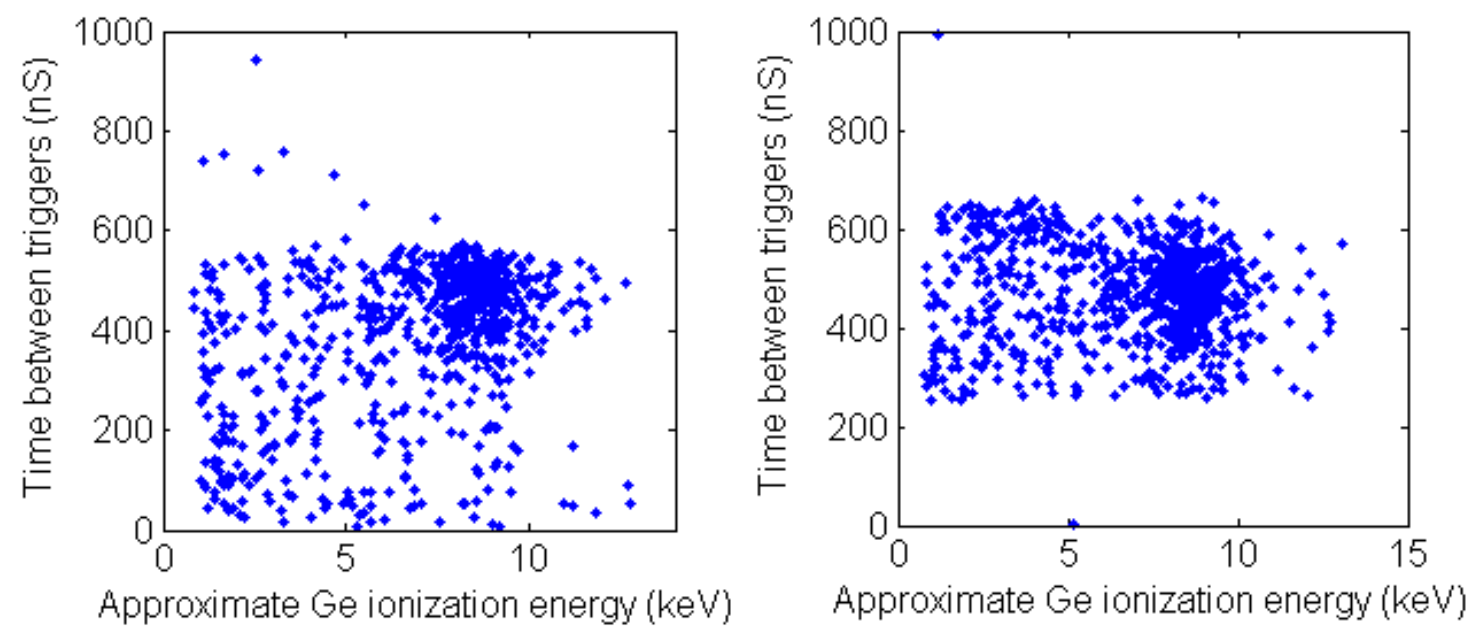

Figure 4.12: Trigger timing data. Left) $\sim 30 \mathrm{keVnr}$ events before fine tuning, showing the ROI population is being excluded at higher ( $>\sim 550 \mathrm{nS})$ trigger spacings. Middle) Data from remainder of run after timing adjustments were implemented. ROI is clearly well defined inside of this parameter space. C) 16keVnr events (after further tuning) showing shifted ionization energy measured. 
Information from the scatter detectors and Ge detector must be carefully routed throughout the hardware in order to appropriately tag and record proper neutron scatter events. While the scatter detectors merely send PMT signal outputs to the logic and timing circuit, the Ge detector's DSP/MCA sends and receives multiple signals to allow neutron tagging and subsequent ionization yield analysis. There are two main paths of signal through the DSP/MCA: the slow channel (cleaner, filtered signal: deals with pulse shaping and energy measurement) and the fast channel (noisier unfiltered signal: deals with timing issues such as count rate, pile-up prevention, etc). The signals utilized in this experiment are the following:

- Analog out - Slow channel signal, trapezoidal shaped pulse for energy measurement (see Figure 4.11).

- ICR - Incoming count rate. Triggers when fast channel energy threshold is exceeded, signaling the start of an event. This is a positive pulse, so it is fed into a TTL $\rightarrow$ NIM converter before entering the timing circuit.

- SCA - Single Channel Analyzer. Triggers when an event is accepted within the energy range of the MCA spectrum.

- Gate - Input on the MCA that alerts it to accept any events being processed while active.

For an event to be accepted by the MCA, one of the scatter detectors must produce a pulse of proper amplitude and timing (relative to the Ge event time, see Section 4.2.3). To determine that these conditions are met, the scatter detector pulse goes through the following processing steps, consisting of NIM electronics (see Figure 4.13 for block diagram). First, each scatter detector's output is fed into its own fanout unit. This allows the signal to be monitored and processed in non-interfering parallel paths. The NaI signals are each then fed into x10 amplifiers (the NE213 


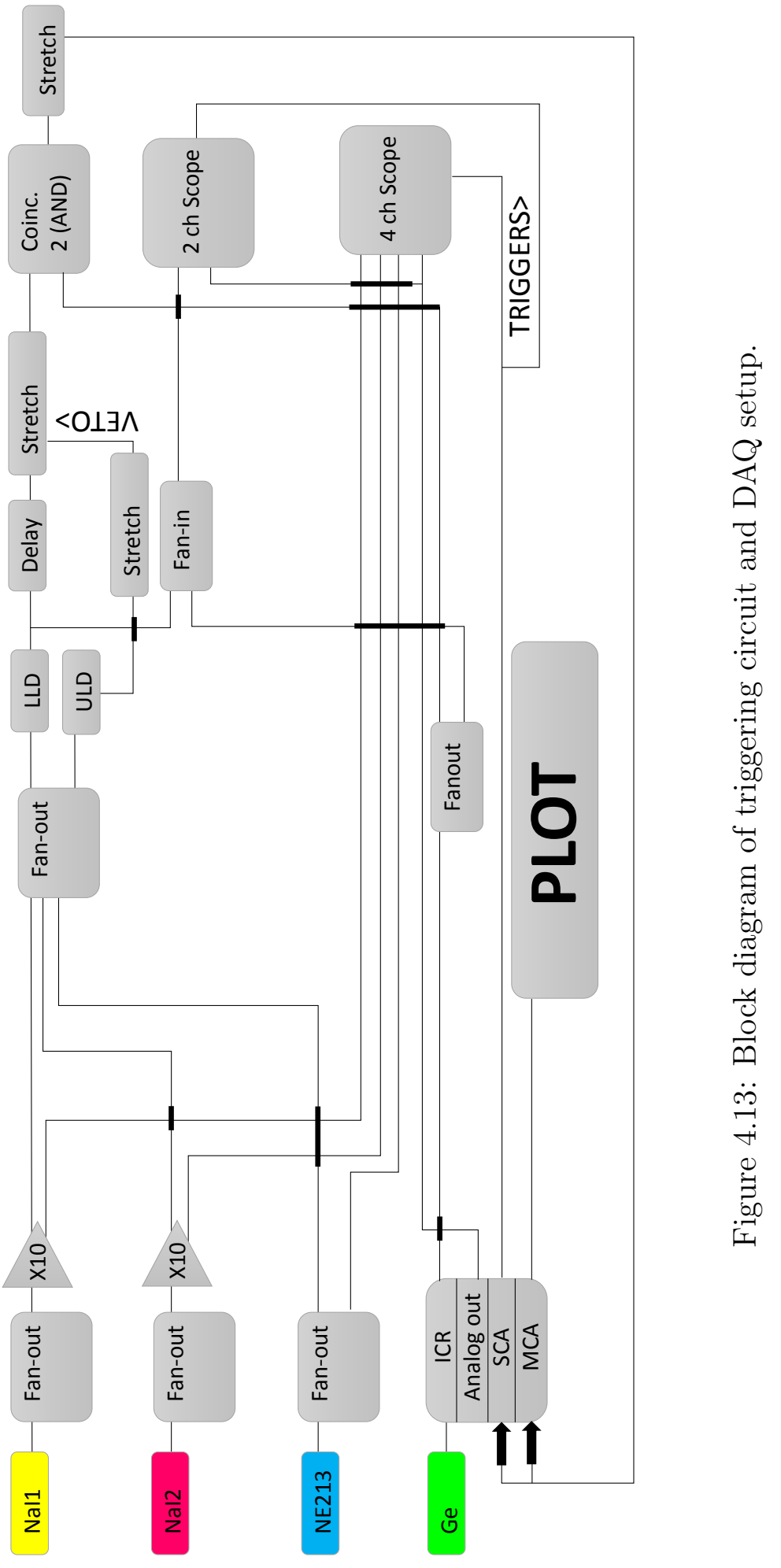


pulses are naturally $\sim 10 \mathrm{x}$ larger, so they do not need amplification). The amplified NaI signals and the raw NE213 signal are then all fed into two different units, a 4 channel oscilloscope with data logging capabilities, and a fan-in/out unit. The 4 channel scope is used to monitor the pulse heights and is triggered upon acceptance of a pulse (at which time it also captures the associated Ge pulse height). The fanin/out combines the 3 detector signals such that they are are handled as one signal down the line. This signal then passes to an LLD and, in parallel, a ULD. The LLD is set high enough to exclude much of the ambient gamma pulses, but not so high to exclude too many neutron hits. The ULD is used as a veto for pulses known to be too high to be relevant. In order for the ULD to veto pulses (which would necessarily have already triggered the LLD during the pulse's initial rise, the ULD logic pulse must be stretched in time, and the LLD logic pulse must be delayed to occur entirely within the ULD veto pulse. As it turns out, this delay is also necessary for scatter event timing, as the ICR pulse arrival is inherently delayed. The amount the ULD is stretched determines the length of the veto and is tuned to block the majority of after-pulsing which often occurs after large pulses. These smaller afterpulses are results of the large pulse event decays and are therefore unwanted in the 'accepted trigger' logic. The un-vetoed, delayed LLD pulses are then stretched to define a window in which they can register coincidence with the ICR pulse from the Ge event. The ICR is also stretched to ensure pulses are long enough to be registered by downstream electronics. The stretched ICR pulses AND delayed, stretched, unvetoed LLD pulses are then fed into a coincidence unit. When any overlap of the two occurs, the unit supplies a short NIM pulse signaling coincidence. This pulse is not long enough to efficiently trigger the gate logic in the MCA, so it is stretched, then fed into the gate input. This signals the MCA to measure and plot the associated Ge pulse. In parallel, the un-altered LLD and ICR pulses are fed into a fan-in, 
combining them into one signal. This signal, as well as the 'analog out' Ge energy signal, is sent into a 2 channel PC oscilloscope (triggered on accepted pulses by the $\mathrm{SCA})$. This is used to monitor the scatter timing parameter alongside the resulting Ge energy. This measurement allows tuning of the pulse stretching and delays as needed (see Section 4.2.3).

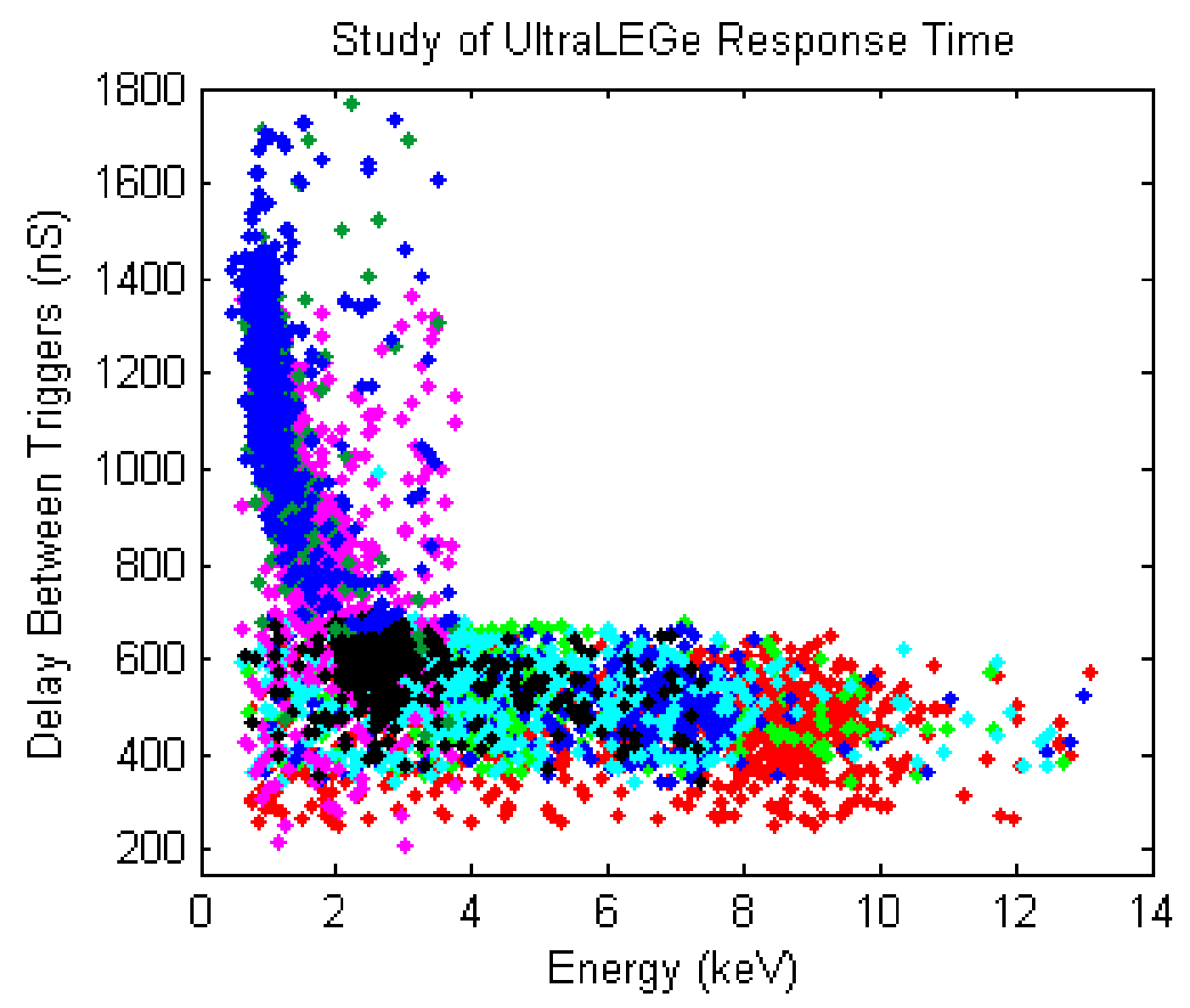

Figure 4.14: Inherent delay of Ge trigger after scatter detector trigger vs. Ge ionization energy. Different colors represent different targeted nuclear recoil energies. Rapid increase of delays with decreasing energy (below $\sim 3 \mathrm{keV}$ ) is unexpected and needs to be accounted for in the timing and logic settings. 


\subsubsection{Timing Deviations}

While the TOFs do not deviate enough $(<10 \mathrm{nS}$ within a $>300 \mathrm{nS}$ acceptance window) to warrant timing compensation, there are other effects in the detector read out process that do require timing compensation. These effects were unknown prior to this analysis and initially caused a great deal of difficulty in the data acquisition process.

Initially, the disparity in detector timing response between the germanium detector and the scatter detectors was analyzed using a ${ }^{22} \mathrm{Na}$ calibration source. The simultaneous $180^{\circ}$ separated gamma events produced from positron-electron annihilation were perfect for characterizing this issue. Based on the delay measured in these events, the signal delays for coincidence condition were then adjusted for the expected neutrons' time of flight. This worked for the higher energy depositions in the Ge, but failed at lower energies, inexplicably requiring the timing parameters to be loosened. Throughout this work, this effect was monitored and can be seen plotted in Figure 4.14. Without this information, precise timing measurements at low Ge energies would not be possible. The cause is likely in the DSP triggering system, with lower energy pulses taking longer to reach the initial trigger threshold, but the effect is larger than one would expect from such an issue.

The effect was studied further using a ${ }^{22} \mathrm{Na}$ source. The simultaneous gamma emissions allowed a 'true' start time to be established using a nearby NaI detector. With this reference time, the timing response of the Ge detector was characterized. Figure 4.15 shows the effect to be largely caused by the low energy shaping filter in the DSP system. However, the study was extended to higher energies (see Figure 4.16) using the 'normal' fast discriminator shaping, showing that the timing disparity is prevalent throughout, regardless of settings. This is a very important effect to 

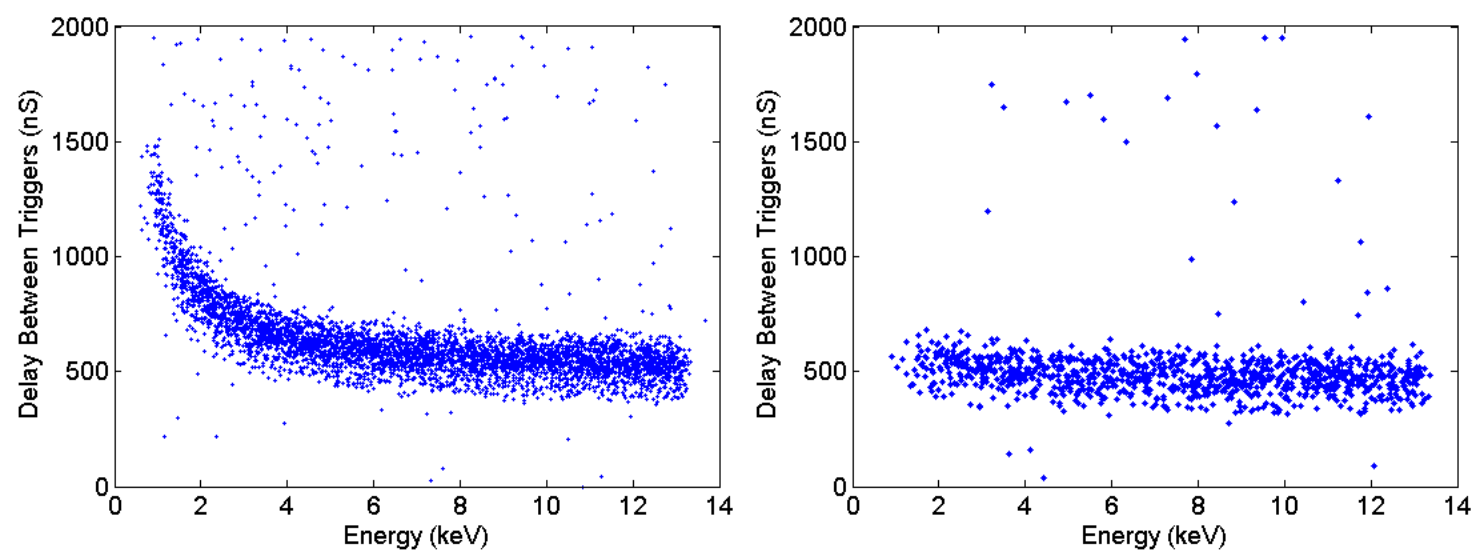

Figure 4.15: Plots showing timing and energy correlation in Ge detector using a ${ }^{22} \mathrm{Na}$ source. Left) Fast discriminator shaping mode set to 'low energy'. Right) Fast discriminator shaping mode set to 'normal'. While trigger efficiency and detector resolution is degraded, timing becomes more uniform.

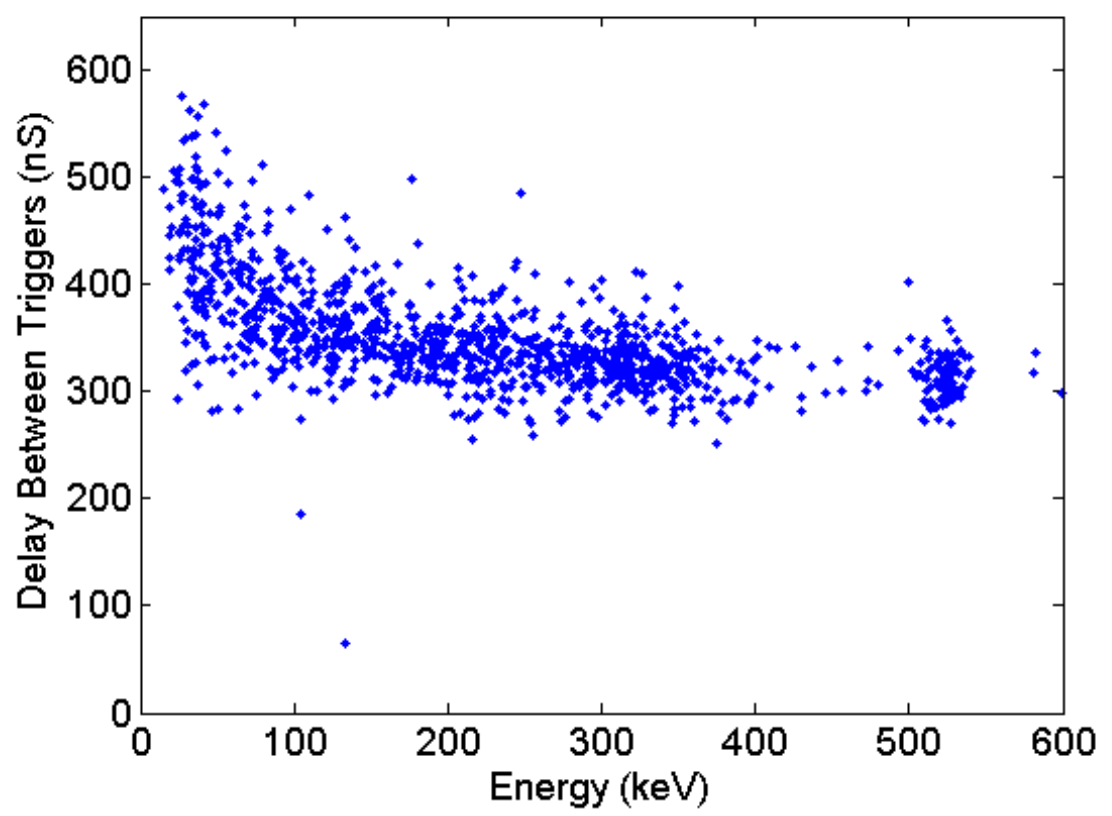

Figure 4.16: Plot showing the timing disparity using higher energy gamma events, including the $511 \mathrm{keV}^{22} \mathrm{Na}$ photo-peak. 
characterize, as any measurements relying on strict timing parameters with such a setup are vulnerable and will suffer unintentional biasing if left uncorrected.

\subsubsection{Scatter Parameter Choices and Future Considerations}

To choose a certain nuclear recoil energy, the neutron energy and tagged scatter angle need to be carefully tuned. Many factors go into this decision. Targeting recoils of $\sim 10 \mathrm{keV}$ in germanium can be done with neutrons of anywhere from $\sim 180 \mathrm{keV}$ to $\mathcal{O}(\mathrm{MeV})$ scale. However, depending on the experimental setup and the physics involved, not all energies are practical in making this measurement.

In choosing the neutron energy, the following factors should be considered:

1. A higher incoming neutron energy means that for a given scatter detector's solid angle, there will be a larger spread in the deposited energies, thus favoring a lower incoming neutron energy.

2. A higher incoming neutron energy results in a higher outgoing neutron energy for a given scatter angle. This leads to higher energy depositions in the scatter detectors which also suffer from reduced sensitivity to nuclear recoils as compared to electronic. This favors a higher incoming neutron energy.

3. The peak in neutron production rate occurs at high (by this experiment's standards) energies, $\sim 550 \mathrm{keV}$ (see Figure 4.17 ). This is a dominant factor in energy choice since this measurement can easily become statistics limited depending how long and at what intensity a beam run can be maintained.

In an ideal world, factor 1 would take precedence, leading to a smaller uncertainty in the measurement. This could be accomplished by fixing the issue with factor 2 . Using more sensitive scatter detectors with good neutron:gamma discrimination (to combat the high background rate of low energy gammas) would serve this purpose. 


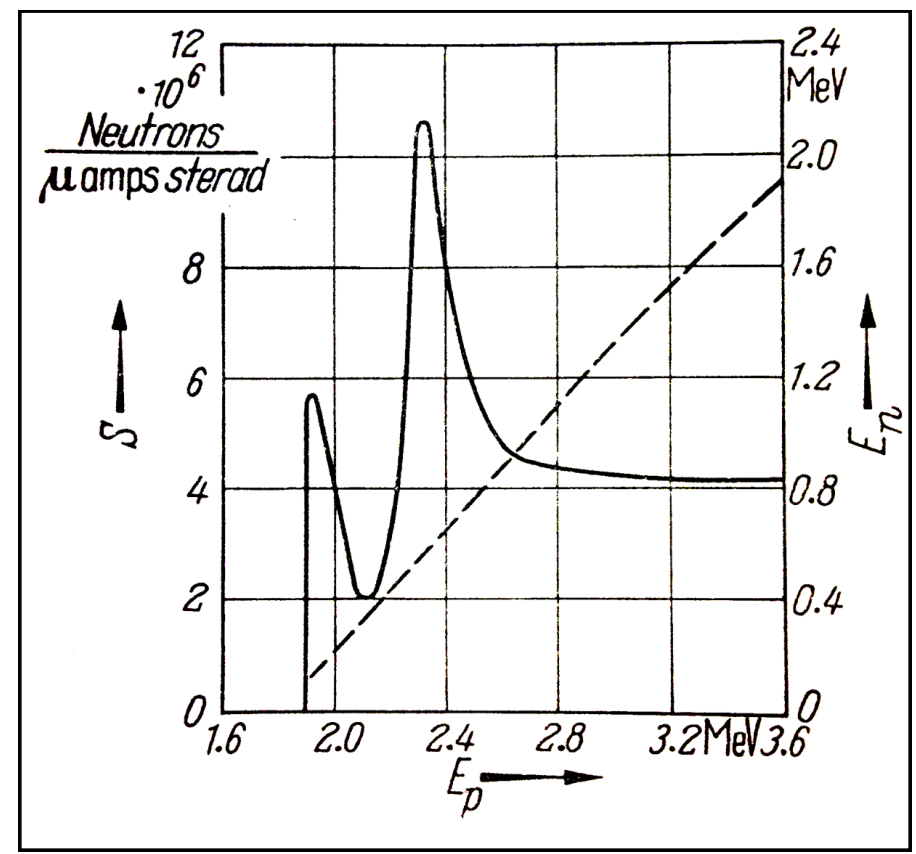

Figure 4.17: Plot of neutron rate (solid line) and energy (dashed) vs. incident proton energy[33]. Note the sharp turn-on at threshold, which is exploited in the energy calibration process.

Improved sensitivity also allows a better neutron tagging rate by accepting lower energy depositions in the scatter detectors. This would negate the use of the higher energy neutrons required in factor 3. These factors are all being considered for future runs using this setup.

In choosing the scatter detectors and placement, the following factors should be considered:

1. Because the target detector area is a region that is quite active in gammas, the scatter detector would benefit greatly from neutron:gamma event discrimination.

2. The scatter detector medium (or its constituent elements) should have a low atomic mass, allowing higher energy transfer from neutron to detector. Al- 


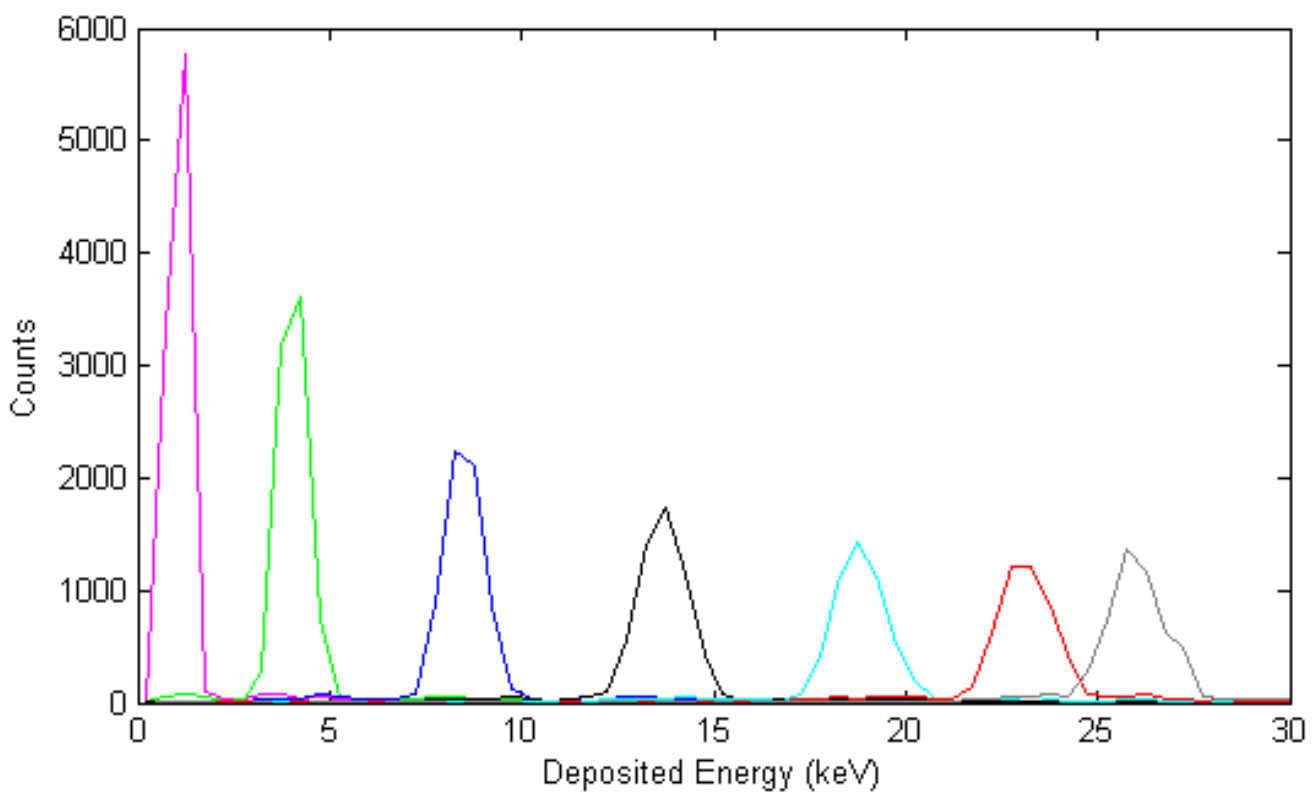

Figure 4.18: Plot of GEANT simulated events scattered at various angles, demonstrating the advantage of shallow angle scatters. Scatter angle in degrees, from left to right: $22.5,45,67.5,90,112.5,135,157.5$

ternatively (or complementarily), the medium could contain elements (such as gadolinium or boron) which benefit from higher cross-sections to neutron interaction.

3. Because the energy deposition scales as $(1-\cos \theta)$ (see Equation 4.4), the effective solid angle (in deposited energy space) scales as $\sin \theta$. Therefore, minimum effective solid angles occur at $0^{\circ}$ and $180^{\circ}$, peaking at $90^{\circ}$. This factor favors extreme angles (near $0^{\circ}$ or $180^{\circ}$ ) for energy resolution purposes, and specifically shallow angles (near $0^{\circ}$ ) for low energy recoils (see Figure 4.18).

4. The kinematics of the elastic scatter prefer forward scattering (see Figure 4.19). Therefore, the data rate is improved at low scatter angles.

5. Perhaps the most obvious factor (other than angle) in the placement of the scatter detectors is the distance at which they are placed from the target detector. 


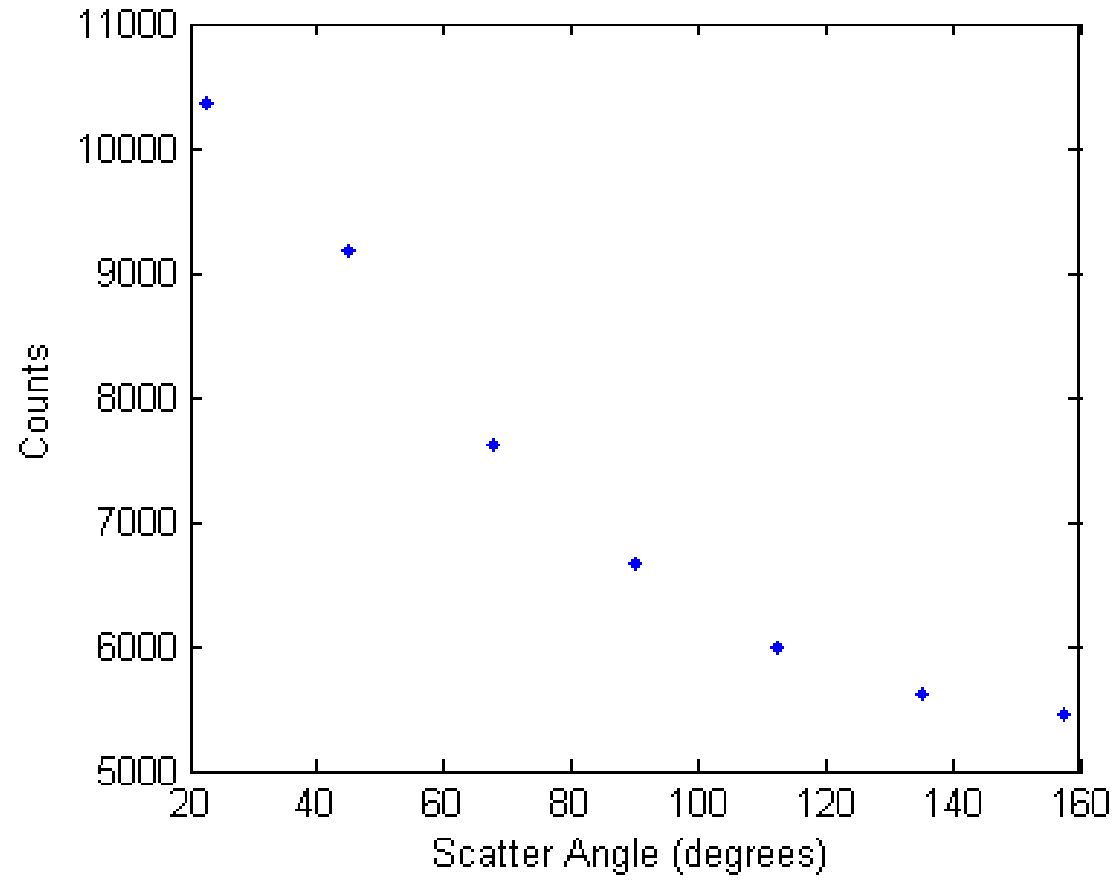

Figure 4.19: Plot of tagged neutron scatters vs. scatter angle, showing shallow angle preference (simulated in GEANT). 


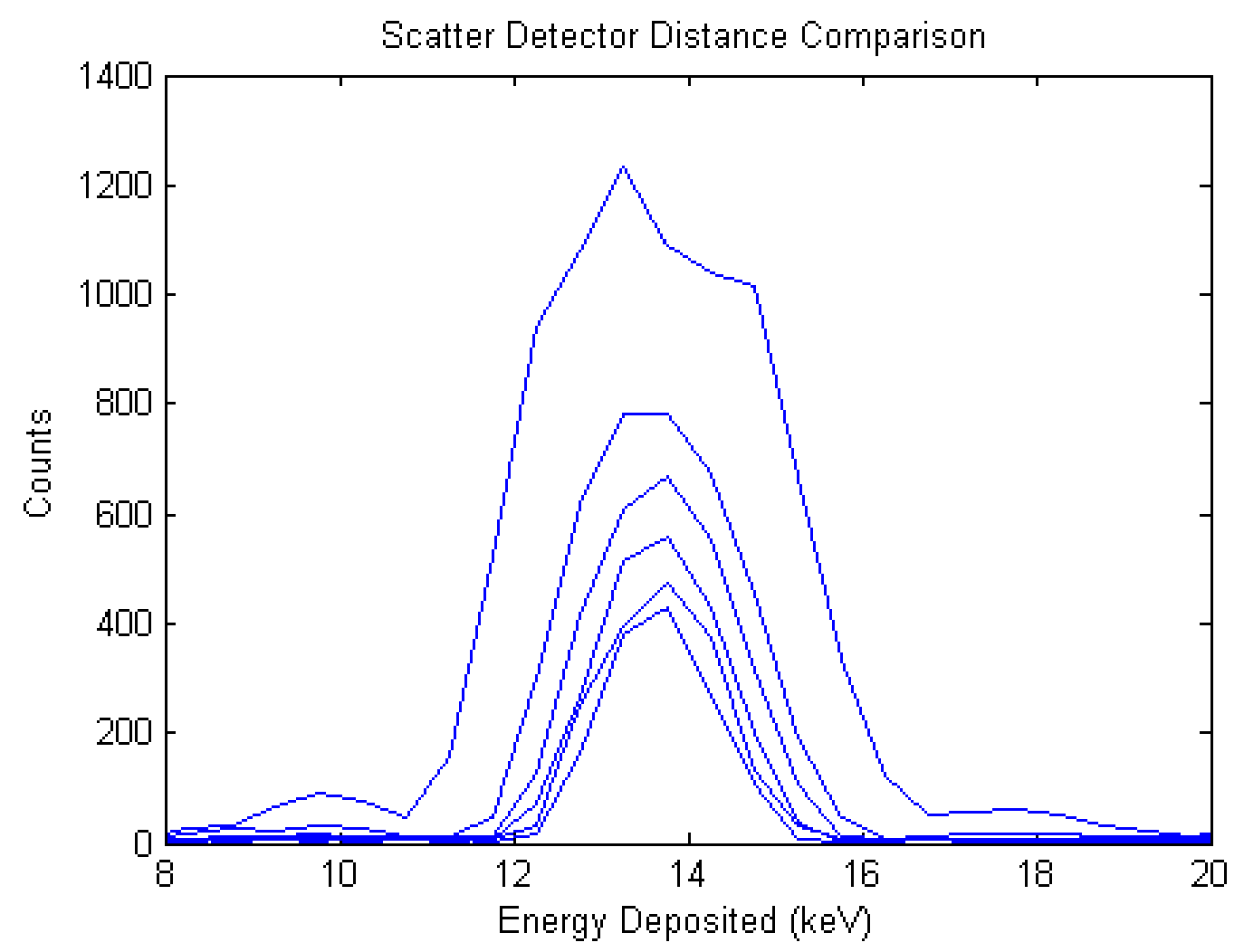

Figure 4.20: Plot of simulated germanium energy deposition spectra from various scatter detector distances, demonstrating the effect on rate and energy spread. Distances in cm, from smallest peak to largest: $34.3,30.5,26.7,22.9,19.1,15.2$

This effect is of course from the resulting solid angle the detector occupies (scaling as $1 / r^{2}$ ). Changing the solid angle occupied influences two important (yet competing) conditions: 1) Reduced solid angle means reduced scatter rate (see Figure 4.20), and 2) Reducing the solid angle reduces the energy spread in the resulting data. A balance must be found between these conditions, and, in the case of this work, that balance was found at a distance of $34 \mathrm{~cm}$.

\subsubsection{Calculating Energy Deposition from Scatter Angle}

For a given incident neutron, the energy it deposits in the medium off of which it elastically scatters (Ultra-LEGe in this case) is represented in the angle at which it 
scatters. This relationship (equation 4.4) is derived kinematically and shown below. In this equation, $\mathrm{E}_{0}$ is the incident neutron energy, $\mathrm{m}$ and $\mathrm{M}$ are the masses of the neutron and Ge nucleus, respectively, and $\theta$ is the angle at which the neutron scatters.

$$
E_{d e p}=E_{0} \frac{2 m M}{(m+M)^{2}}\left(1-\cos \theta_{c m}\right)
$$

It should be noted that equation 4.4 is in terms of $\theta_{c m}$. This is the scatter angle in the center of mass frame of the neutron-germanium collision. The relation between the center of mass frame angle $\theta_{c m}$ and the lab angle is shown in equation 4.5.

$$
\tan \theta_{l a b}=\frac{\sin \theta_{c m}}{m / M+\cos \theta_{c m}}
$$

It is clear from equation 4.5 that when $\mathrm{m}<<\mathrm{M}, \theta_{l a b} \approx \theta_{c m}$. This is the case for a target mass of germanium. However, the two angles are not equal, and the correction, although minor (see Table 4.1), has been implemented in this work. The correction peaks at $\theta=90^{\circ}$ and increases with decreasing target nucleus mass. These factors should be considered in future experiments.

\begin{tabular}{|l||l|l|l|l|l|l|}
\hline Lab Frame Angle (degrees) & 45 & 51 & 58 & 66 & 87 & 145 \\
\hline Center of Mass Frame Angle (degrees) & 45.6 & 51.6 & 58.7 & 66.7 & 87.8 & 145.5 \\
\hline
\end{tabular}

Table 4.1: Lab frame angles used in this work and their corresponding center of mass frame angles.

\subsection{Uncertainties}

There are many sources of uncertainty in this experiment, those deemed significant enough to deserve analysis are the following: 
- Incident neutron energy

- Angular placement and solid angle of detectors

- Resolution of germanium detector

These have been carefully considered in the analysis of this data, and described in detail in the following sections. While there are many sources of uncertainty, it should be noted that only two of these factors have the possible effect of shifting the data: 1) Proton beam uncertainty (bending magnet stability), and 2) Detector placement. It will be shown, that these two factors have negligible errors in this work. The remaining uncertainties instead result in a spread of the measured energy, leaving the accuracy of the peak value intact.

\subsubsection{Uncertainty in Neutron Energy}

One of the claims in this work is that the beam stability and experiment design allow it to probe very specific neutron energies for nuclear recoil calibrations. The reasons for this being the following:

- Proton beam stability

- Small energy loss in LiF target

- Small spread in energy throughout germanium detector solid angle

- Precise threshold calculation

The proton beam stability results from two main factors, the bending magnet stability, and the ability of the terminal potential to be automatically compensated in real-time. The bending magnet stability can be seen in Figure 4.21. This figure shows the bending field, left alone at $\sim 275.41 \mathrm{mT}$ for 20 minutes. In this period it exhibited a maximum deviation of $10 \mu \mathrm{T}$. At the energies used in this experiment, this would equate to a $0.027 \%$ change in neutron energy. Over the period of longer runs 


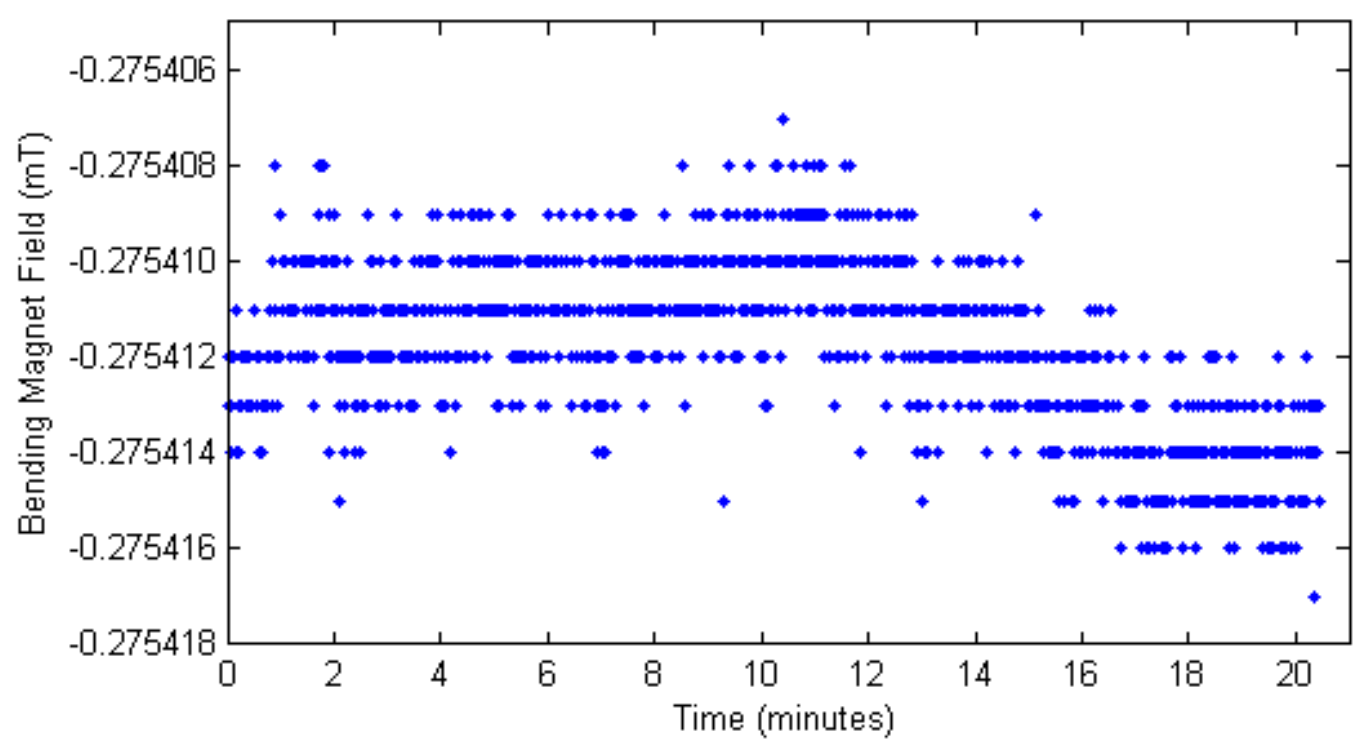

Figure 4.21: Bending magnet field over time. 0.00363\% (10 $\mu \mathrm{T})$ maximum deviation in 20 Minutes.

( 8-10hrs), the effect is increased, but by no more than a factor of $3(0.081 \%$ change in neutron energy, worst case). The ability of the terminal potential stabilizer to lock in on the appropriate voltage to maintain a centered beam downstream allows full appreciation of the magnet stability, allowing negligible spread in the proton beam energy.

When the protons encounter the LiF target, some interact immediately, producing neutrons (corresponding to the incident proton beam energy). However, some will interact with the LiF film, losing energy before undergoing the ${ }^{7} \operatorname{Li}(p, n)^{7}$ Be reaction. In this case, the outgoing neutron energy corresponds to the reduced proton energy. For this experiment, the $\mathrm{LiF}$ used is chosen to be thin $(75 \mathrm{~nm})$ to reduce this effect (see Figure 4.22). This thickness equates to a spread in the proton energy of $\sim 2.5 \mathrm{keV}$ for the neutron threshold calibration and even less for the data runs (at higher proton energies). This spread in proton energy at the energies used here equates to a $0.26 \%$ 


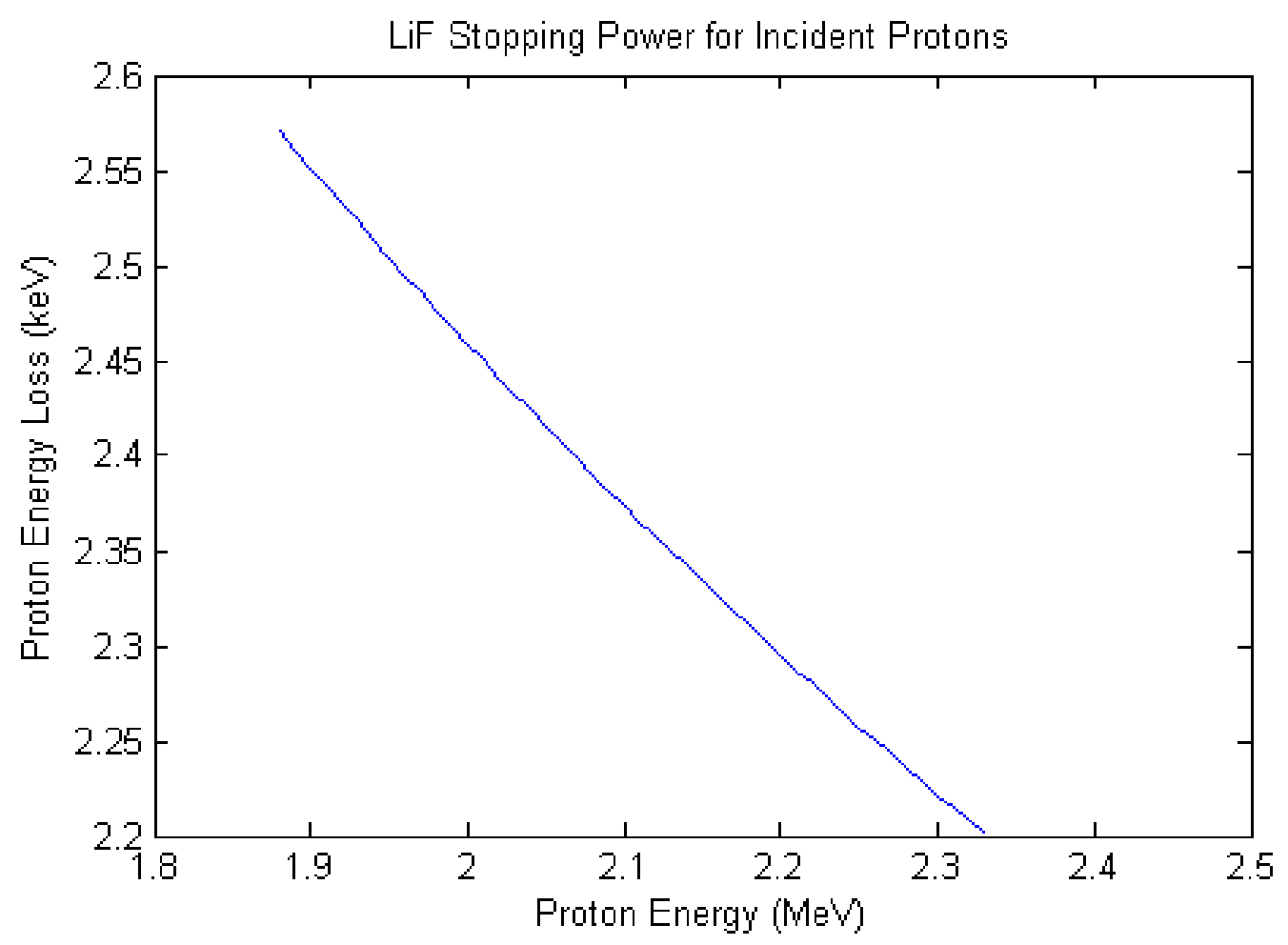

Figure 4.22: Stopping power of LiF for incident protons. This plot shows all proton energies used in this work (see Section 4.3.1). Stopping power values provided by NIST pstar database [35].

spread in neutron energy. Again, this is a negligible amount for this measurement.

Since the neutron energies emitted are angle dependent, the solid angle of the germanium detector equates to a spread in neutron energy (see Figure 4.6). In this setup, the germanium detector occupies $\sim 6^{\circ}$ of the neutron 'cone'. This results in a $\sim 2.3 \mathrm{keV}$ spread $(0.4 \%)$ in neutron energy. While it is the most significant of these factors, it is still negligible in this experiment.

The neutron production threshold calibration precision is essential for this experiment. However, due to the stability of the proton beam, sufficient precision is easily achievable. As seen in Figure 4.2, which was not a particularly rigorous calibration (almost a worst-case in fact), the threshold was found to within $21 \mu \mathrm{T}$. This equates 
to $0.064 \%$ uncertainty in the resulting neutron energy.

\subsubsection{Scatter Angle Uncertainties}

There are three main sources of uncertainty in defining the neutrons' scatter angles for a given run.

1. Physical placement of the detectors relative to the beam

2. Scatter detector solid angle

3. Germanium detector solid angle

An angle is chosen for each run, which then must be physically replicated in the lab setup. This is performed using two metal meter-sticks bolted together at the end to allow free choice of angle which can then be locked in place. This fixture is then suspended with one of the arms on a string which is itself suspended above (and parallel to) the beam line. The other arm is then suspended from the ceiling directly above, ensuring it is not tugged in any horizontal direction. This fixture allows for a sufficiently precise alignment of the scatter detectors to the germanium detector and beam axis. From visual estimation, $\leq 1 \mathrm{~cm}$ of error is expected in this alignment, resulting in an error of $\sim 1.6^{\circ}$ in scatter angle. At $90^{\circ}$ (worst case), this equates to a $1.4 \%$ shift in energy.

The scatter detectors occupy $\sim 5 \mathrm{~cm}$ perpendicular to $\theta$. This equates to an $8.4^{\circ}$ spread in $\theta$. This has the largest effect in the measurement at $\theta=90^{\circ}$, due to the $\mathrm{E}_{d e p}=\alpha(1-\cos \theta)$ relationship (see Section 4.2.6). Therefore, even though the solid angle stays the same, the effective solid angle (in energy deposition space) varies for each choice of angle. In this work, the worst spread occurs for the two data points taken at $87^{\circ}$. In those instances, the full detector diameter equates to a $15.5 \%$ spread in energy values. At the least affected angle used, $145^{\circ}$, the spread is $5.1 \%$. This effect is mitigated by the use of polyethylene collimating wedges (see Figure 4.8). 
These serve the purpose of narrowing the resulting germanium spectra peaks by reducing neutron hits at the extreme angles of the detector face, cutting the angular occupation in half while maintaining statistics at the central region. While it does not completely shield those areas, the effect is still present, as seen in Figure 4.9.

The fact that the germanium occupies a significant solid angle of the beam causes additional spreading in the energy spectra. In the setup used here, the detector occupies $\sim 6.1^{\circ}$. At $87^{\circ}$ (worst case), this equates to a $5.3 \%$ spread in energy values $\left(3.1 \%\right.$ at $\left.145^{\circ}\right)$.

\subsubsection{Germanium Detector Resolution}

The resolution of the germanium detector is approximately linear in this range, with the full-width half-max approximately matching the following equation (determined from calibration):

$$
F W H M=0.113 e V+0.005 E(k e V)
$$

For the worst case in this work, this results in a FWHM of $154 \mathrm{eV}(\sigma \approx 67 \mathrm{eV})$ for the highest energy data point. This peak is at $8.1 \mathrm{keV}$, meaning the sigma is $0.83 \%$ of the peak energy. At the lowest energy data point, the detector's FWHM is $133 \mathrm{eV}$ $(\sigma \approx 58 \mathrm{eV})$. This peak is at $0.7 \mathrm{keV}$, so the sigma is $8.3 \%$ of the peak energy.

\subsubsection{Uncertainties Condensed}

- Uncertainty in neutron energy

- Proton beam stability*,$<0.081 \%$

- Energy loss in $\mathrm{LiF}^{*}, \leq 0.26 \%$

- Germanium detector solid angle, 0.4\%

- Threshold calibration*,$\leq 0.064 \%$ 


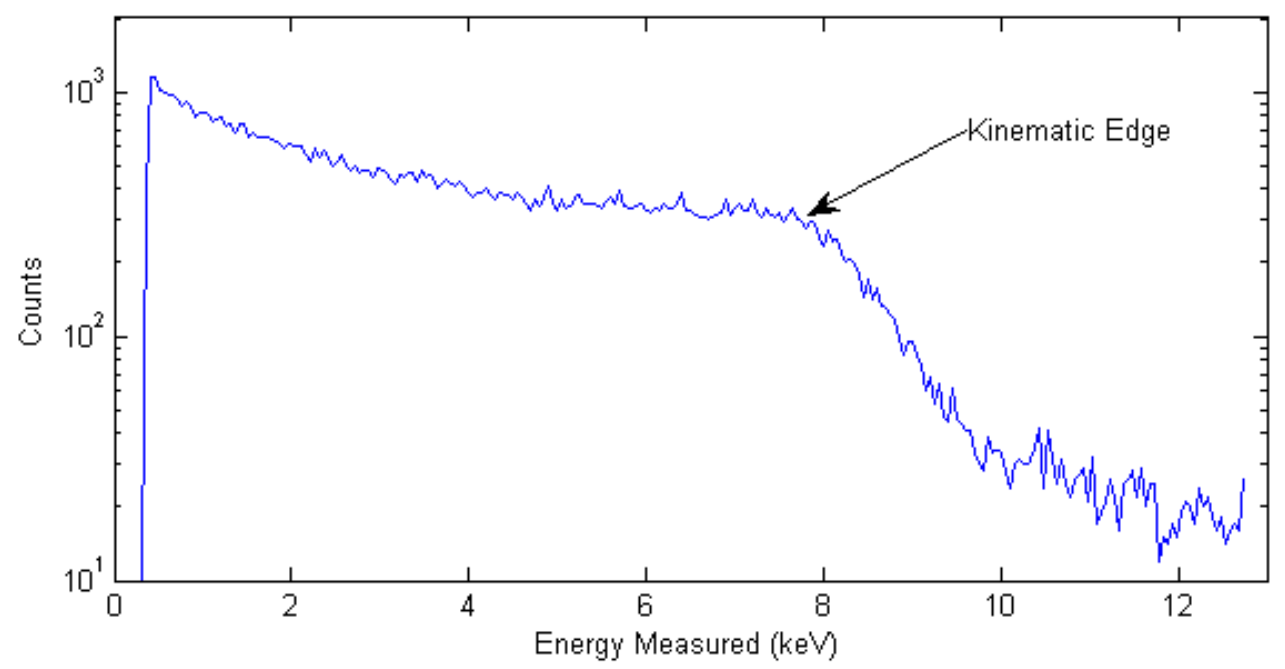

Figure 4.23: Full spectrum of measured event energies. This is the spectrum obtained by recording all events (no scatter detector requirement. The neutron shelf can easily be seen, marked by the endpoint at which $180^{\circ}$ scatters have occurred. This is the expected shape of the background due to random coincidence events.

- Detector positioning and solid angles

- Physical placement*,$\leq 1.4 \%$

- Scatter detector solid angle, worst $=15.5 \%$, best $=5.1 \%$

- Germanium detector solid angle, worst $=5.3 \%$, best $=3.1 \%$

- Germanium detector resolution, worst $=8.3 \%$, best $=0.83 \%$

*Indicates factors that are not accounted for in simulations.

\subsection{Analysis and Results}

The data obtained in this experiment comes in the form of a spectral peak on top of a background, a common situation in this field. Background events can be registered due to random coincidence with scatter detector events. The background shape is dominantly composed of the full spread of neutron energy depositions in the germanium detector. This spectrum is in the form of a shelf with an upper limit, 
the so-called 'kinematic edge' at which $180^{\circ}$ scatters deposit the maximum energy (see Figure 4.23). Events above this energy (further than detector resolution would explain) can be explained by multiple scatters or photons, but are rare in comparison.

In order to define a proper fit for these spectra, an appropriate background shape should be added to an appropriate peak shape. The background shape described above is quite complex and difficult to form a fit around. Unfortunately, simulation data is unable to be used as it relies on the data itself (Lindhard factor). This fit will, however, be attempted in future analyses for publication. In addition to fitting the background, an appropriate fit for the peak must be constructed. A rigorous fit for this feature is complicated by the following:

- The geometry of the scatter detector

- The effect of the polyethylene collimator

- The asymmetrical 'lossy' Gaussian due to incomplete energy collection

- Detector resolution effects

- Detector efficiencies at low energy data points

The complexity of this fit precluded its use in this work, but will be attempted in analyses for future publication. Instead, a simpler model was chosen, and it is argued here that even with conservative estimates of the error introduced, the measurement is robust and scientifically relevant. It should be noted that the two data points of lowest energies are known to be skewed by detector threshold effects. These will be modeled and accounted for in future publication, and are expected to lower the peak energies of these data points.

It has been found that a double Gaussian was able to fit the date quite effectively, so this approach was used for the data presented here. Mean and sigma values for the peak measurements were quite robust to background subtraction as the dominant 
background shape is fairly flat. For this reason, the data extracted is not expected to deviate significantly with a more rigorous, scientifically motivated fit. The values obtained from these fits can be found in Table 4.2. The raw data along with the fits, parameters, and errors can be found at the end of this section. The data is shown compared to the Lindhard model (best fit k-value) in Figures 4.24 and 4.25. The Lindhard equation (ionization efficiency as function of recoil energy) for a given atomic number $\mathrm{Z}$, mass number $\mathrm{A}$, and recoil energy $\mathrm{E}_{r}$ is defined as the following:

$$
\varepsilon=\frac{k * g(\epsilon)}{1+k * g(\epsilon)}
$$

where

$$
\begin{gathered}
k=0.133 Z^{2 / 3} A^{-1 / 2} \\
g(\epsilon)=3 \epsilon^{0.15}+0.7 \epsilon^{0.6}+\epsilon \\
\epsilon=11.5 E_{r} Z^{-7 / 3}
\end{gathered}
$$

Using this model, a best-fit $\mathrm{k}$-value of $\mathrm{k}=0.146$ was found. The raw data from each run is shown along with its fit in Figures 4.26 through 4.35 . 


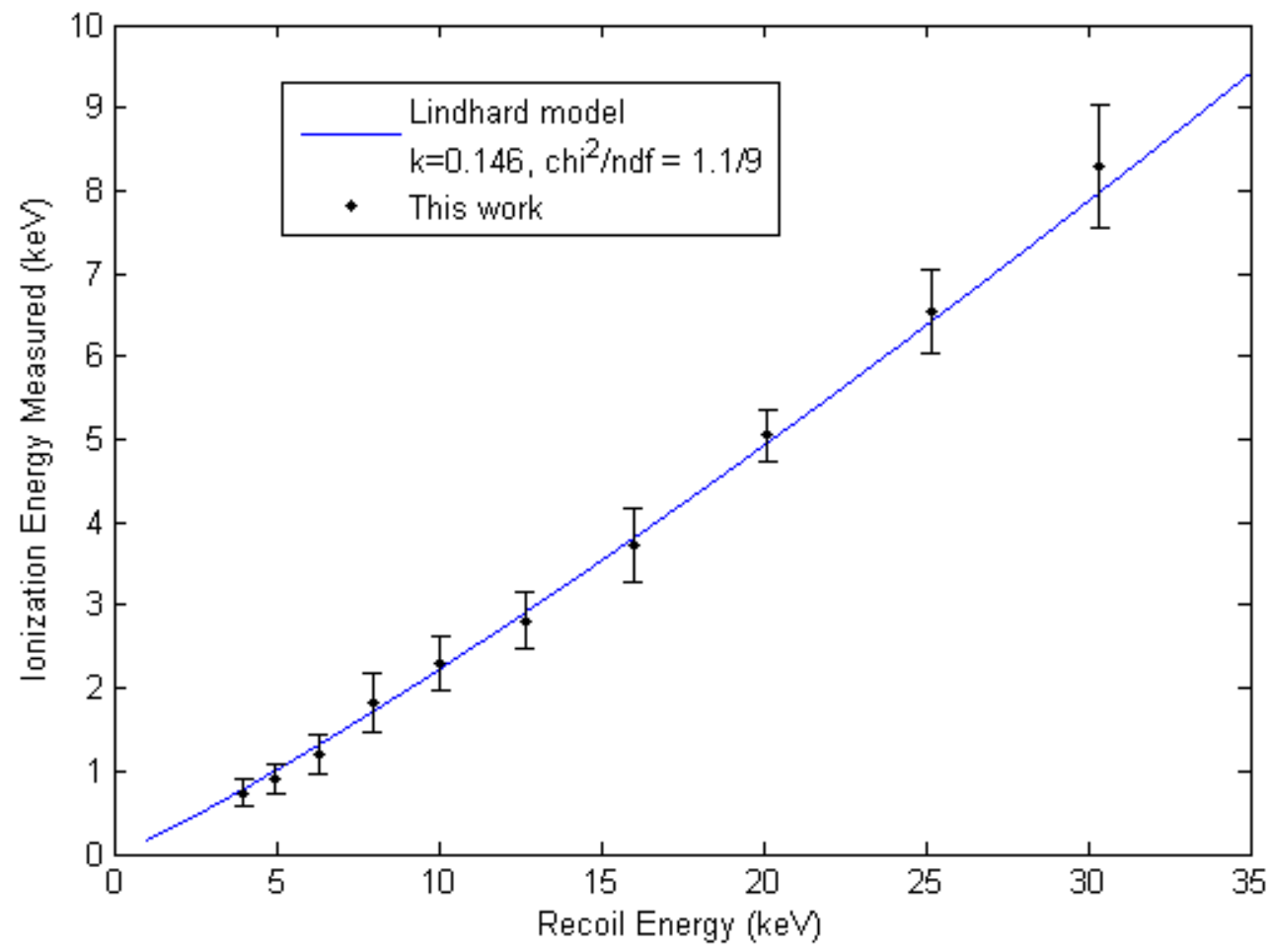

Figure 4.24: Ionization measurements at 10 different recoil energies, using the sigma values of the Gaussian fits for the errors, a conservative approach.

\begin{tabular}{|c|c|c|c|c|c|}
\hline $\mathrm{E}_{n}(\mathrm{keV})$ & $\theta_{\text {lab }}\left({ }^{\circ}\right)$ & $\mathrm{E}_{\text {dep }}(\mathrm{keV})$ & $\mathrm{E}_{\text {meas }}(\mathrm{keV})$ & $\sigma_{E}(\mathrm{keV})$ & Ion. \% \\
\hline 600 & 40 & 3.99 & 0.74 & 0.186 & 0.186 \\
600 & 45 & 4.98 & 0.90 & 0.181 & 0.181 \\
600 & 51 & 6.30 & 1.20 & 0.190 & 0.190 \\
600 & 58 & 7.98 & 1.82 & 0.365 & 0.228 \\
600 & 66 & 10.05 & 2.31 & 0.330 & 0.230 \\
476 & 87 & 12.68 & 2.81 & 0.344 & 0.222 \\
601 & 87 & 16.01 & 3.72 & 0.452 & 0.232 \\
398 & 145 & 20.14 & 5.05 & 0.308 & 0.251 \\
498 & 145 & 25.17 & 6.54 & 0.513 & 0.260 \\
601 & 145 & 30.37 & 8.29 & 0.740 & 0.273 \\
\hline
\end{tabular}

Table 4.2: Data from all runs used in this analysis. Variables (left to right): incident neutron energy, scatter angle (lab-frame), energy deposited by nuclear recoil, energy measured, sigma value of Gaussian peak-fit, and \% ionization energy measured. 


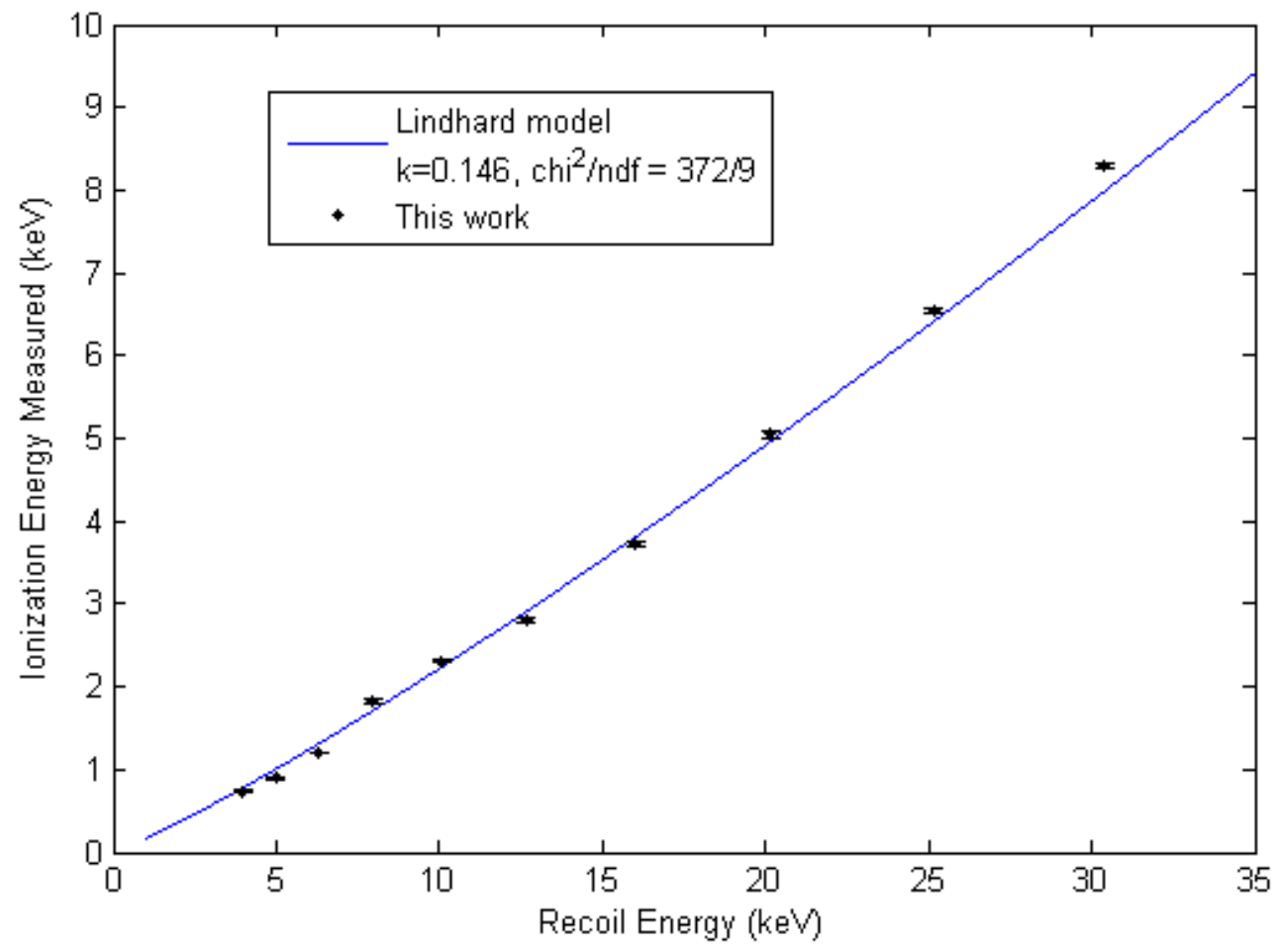

Figure 4.25: Ionization measurements at 10 different recoil energies, using the statistical uncertainty in the mean for the errors. This method does not account for systematic effects. 


$$
\mathrm{E}_{\text {dep }}=4.0 \mathrm{keV}
$$

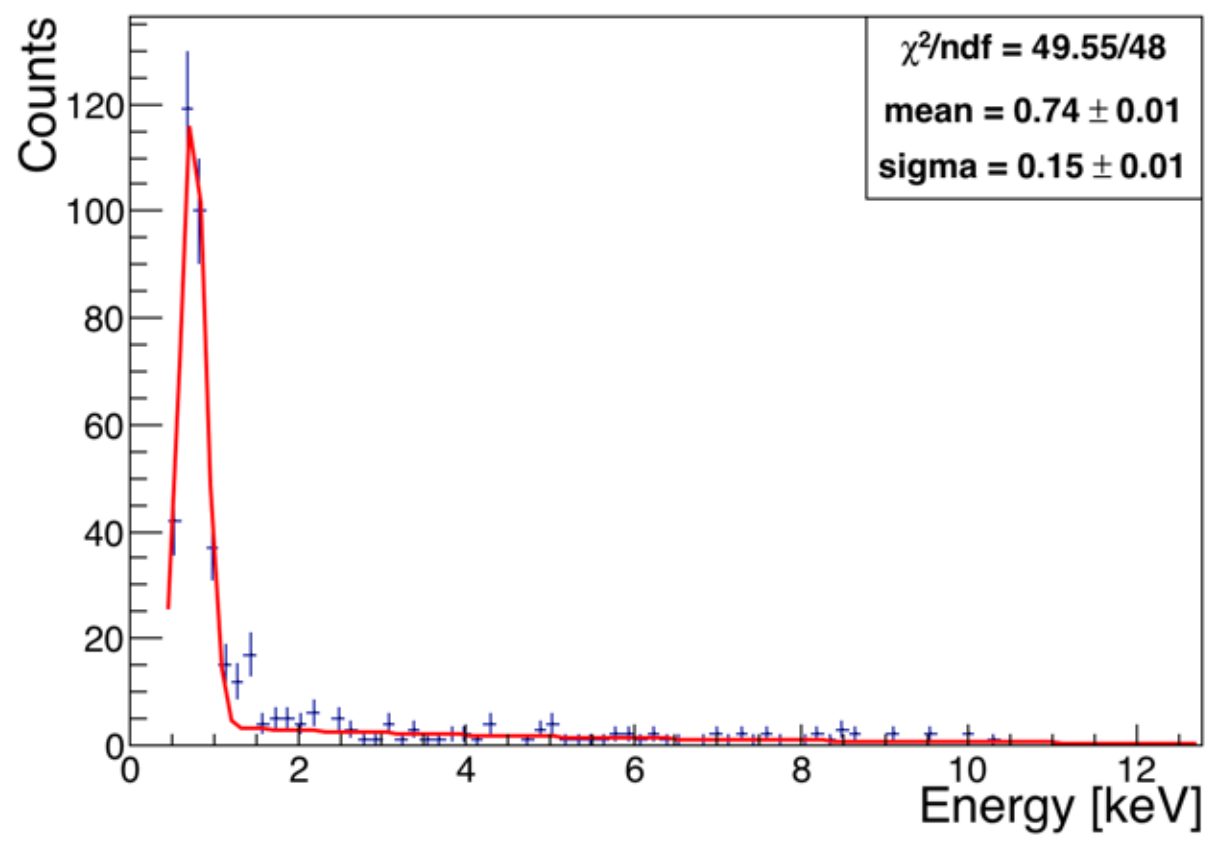

Figure 4.26: Raw data and fit for $4.0 \mathrm{keV}$ nuclear recoil depositions.

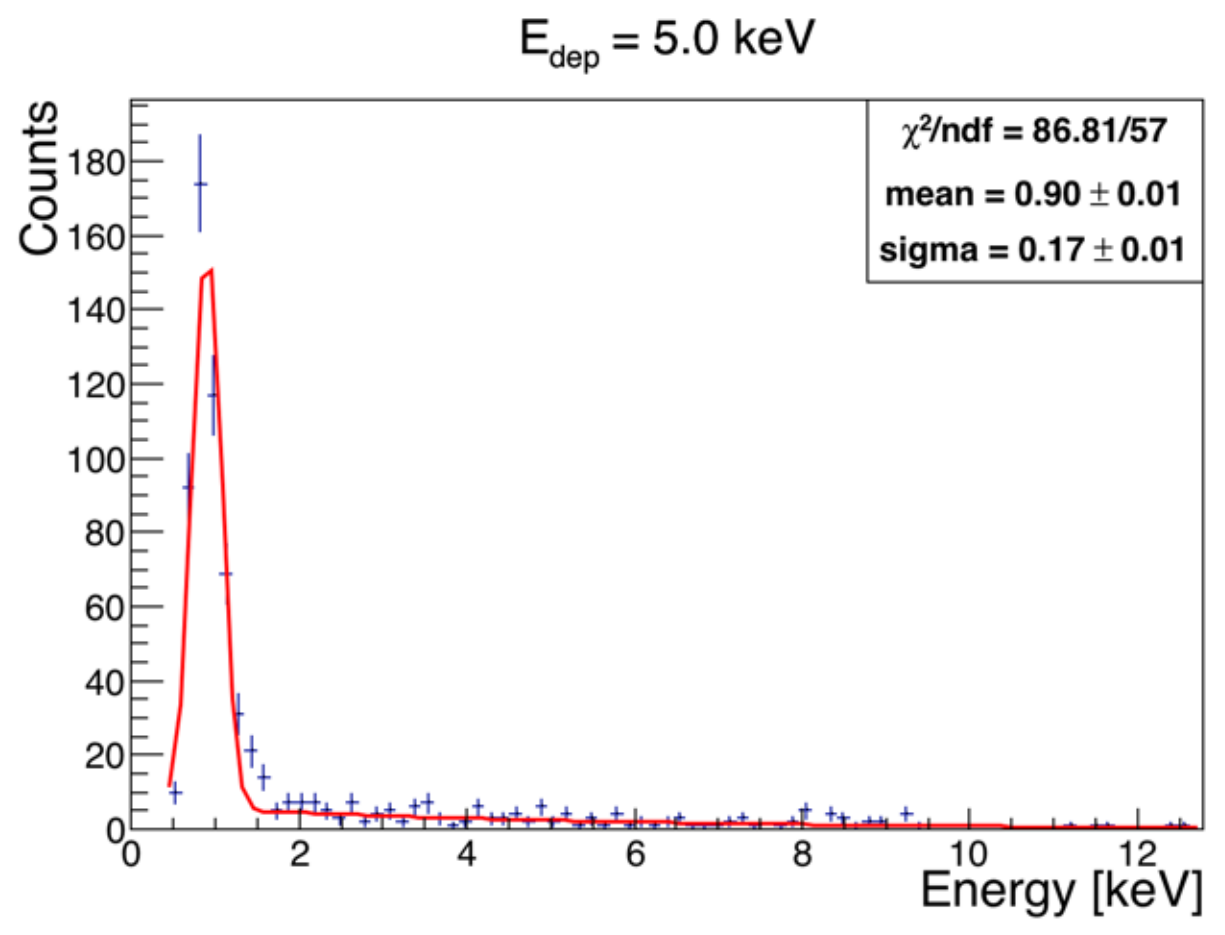

Figure 4.27: Raw data and fit for $5.0 \mathrm{keV}$ nuclear recoil depositions. 


$$
\mathrm{E}_{\text {dep }}=6.3 \mathrm{keV}
$$

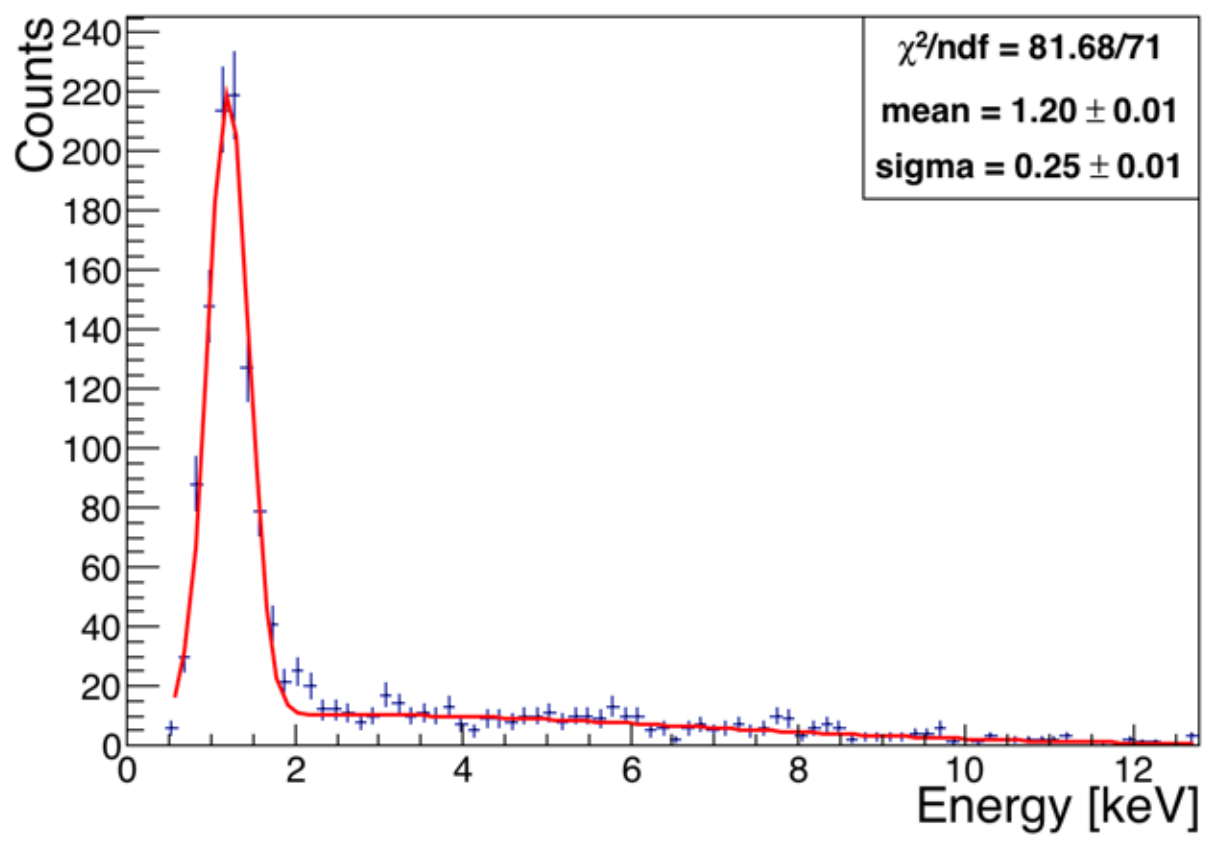

Figure 4.28: Raw data and fit for $6.3 \mathrm{keV}$ nuclear recoil depositions.

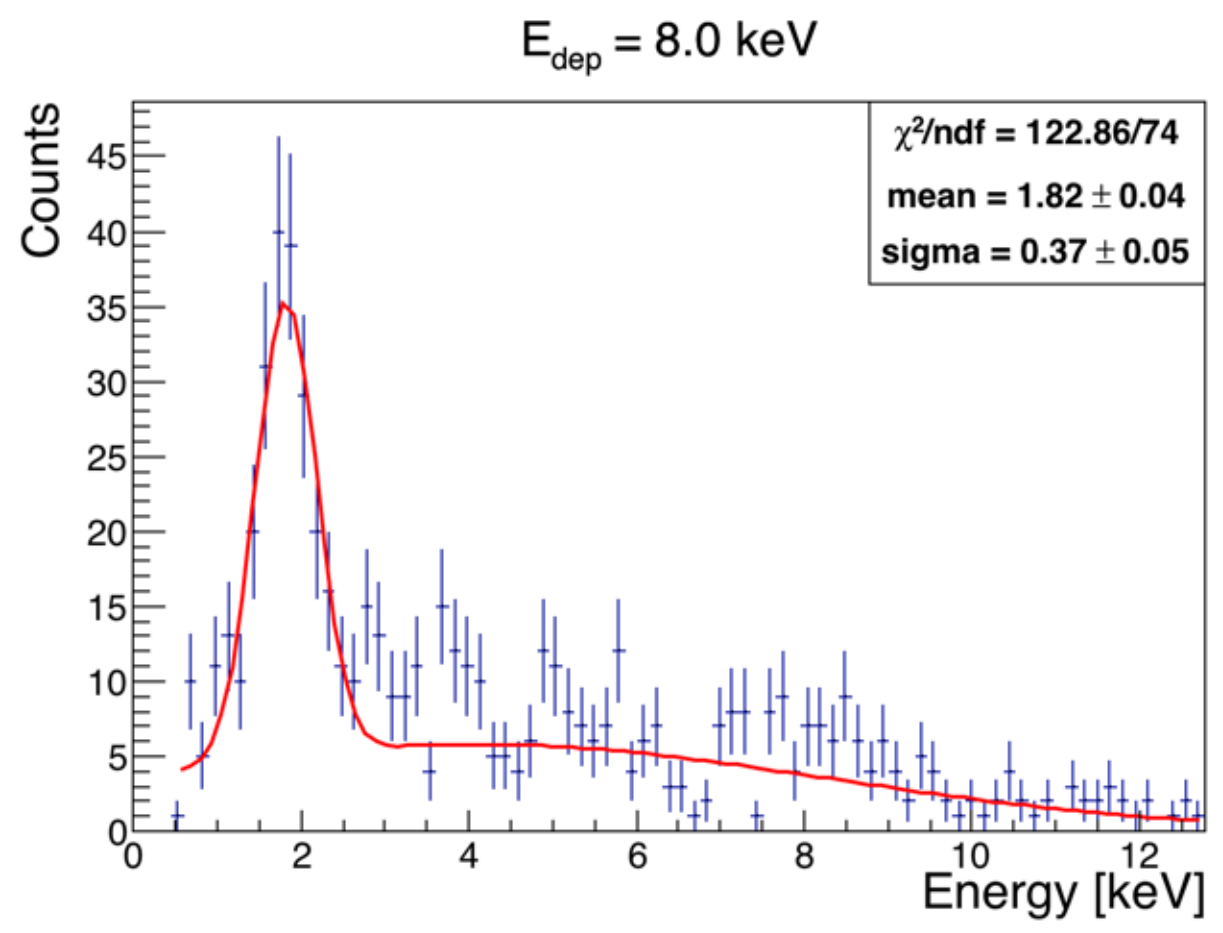

Figure 4.29: Raw data and fit for $8.0 \mathrm{keV}$ nuclear recoil depositions. 
$E_{\text {dep }}=10.1 \mathrm{keV}$

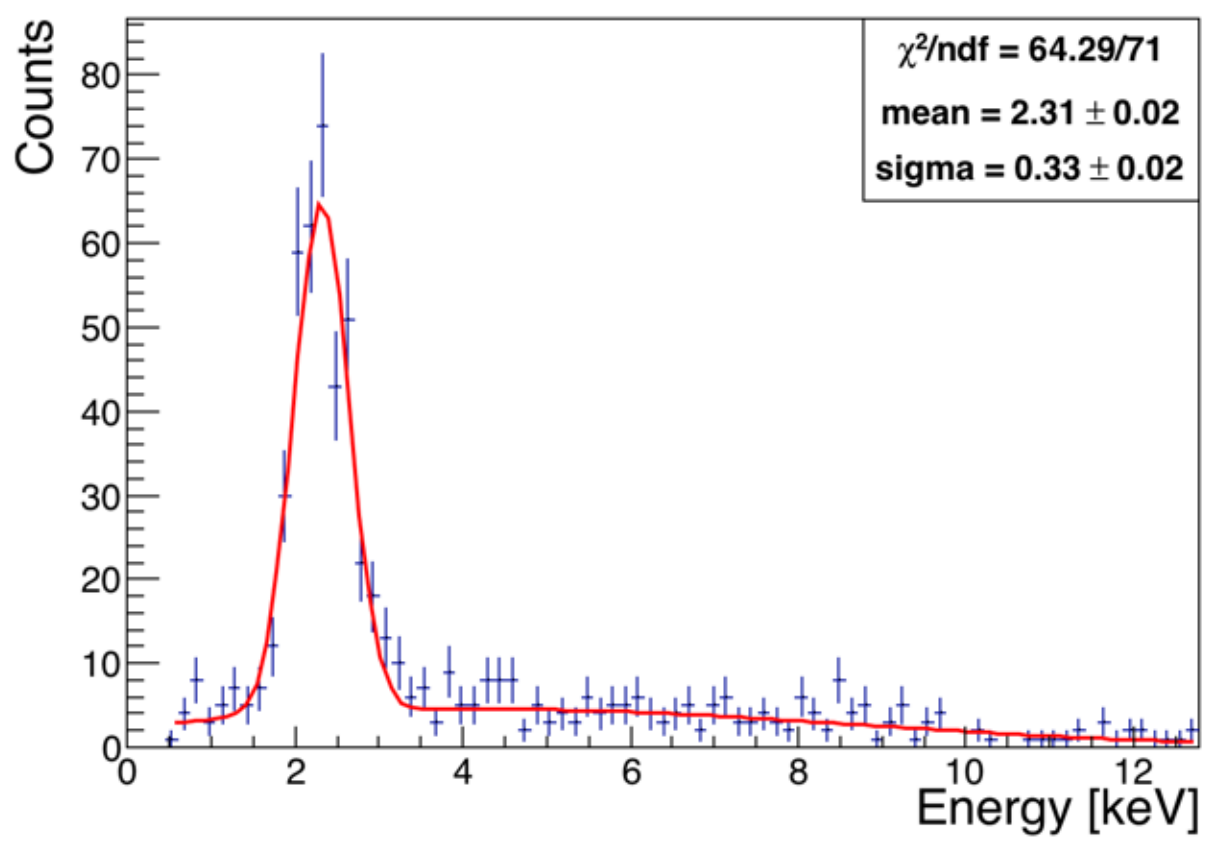

Figure 4.30: Raw data and fit for $10.1 \mathrm{keV}$ nuclear recoil depositions.

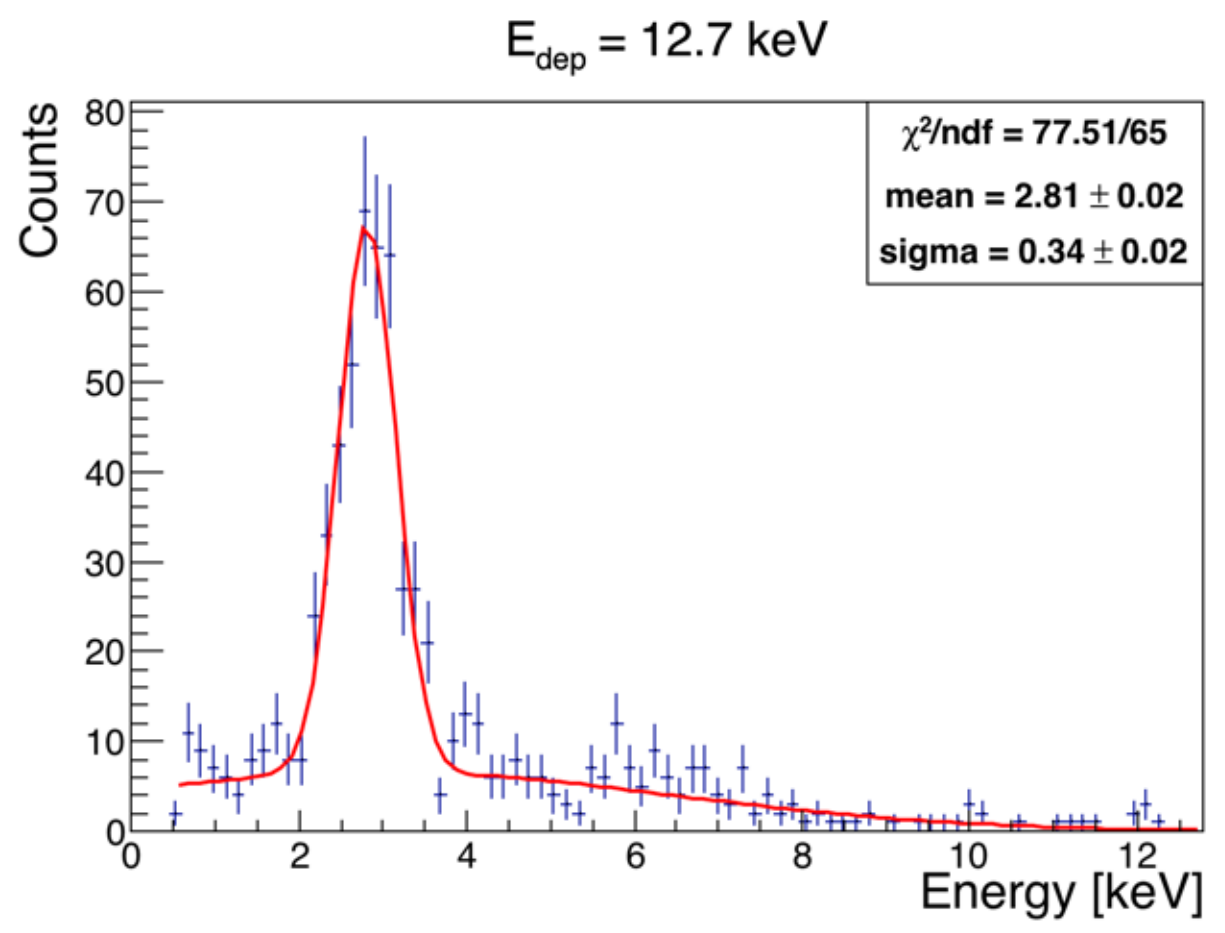

Figure 4.31: Raw data and fit for $12.7 \mathrm{keV}$ nuclear recoil depositions. 


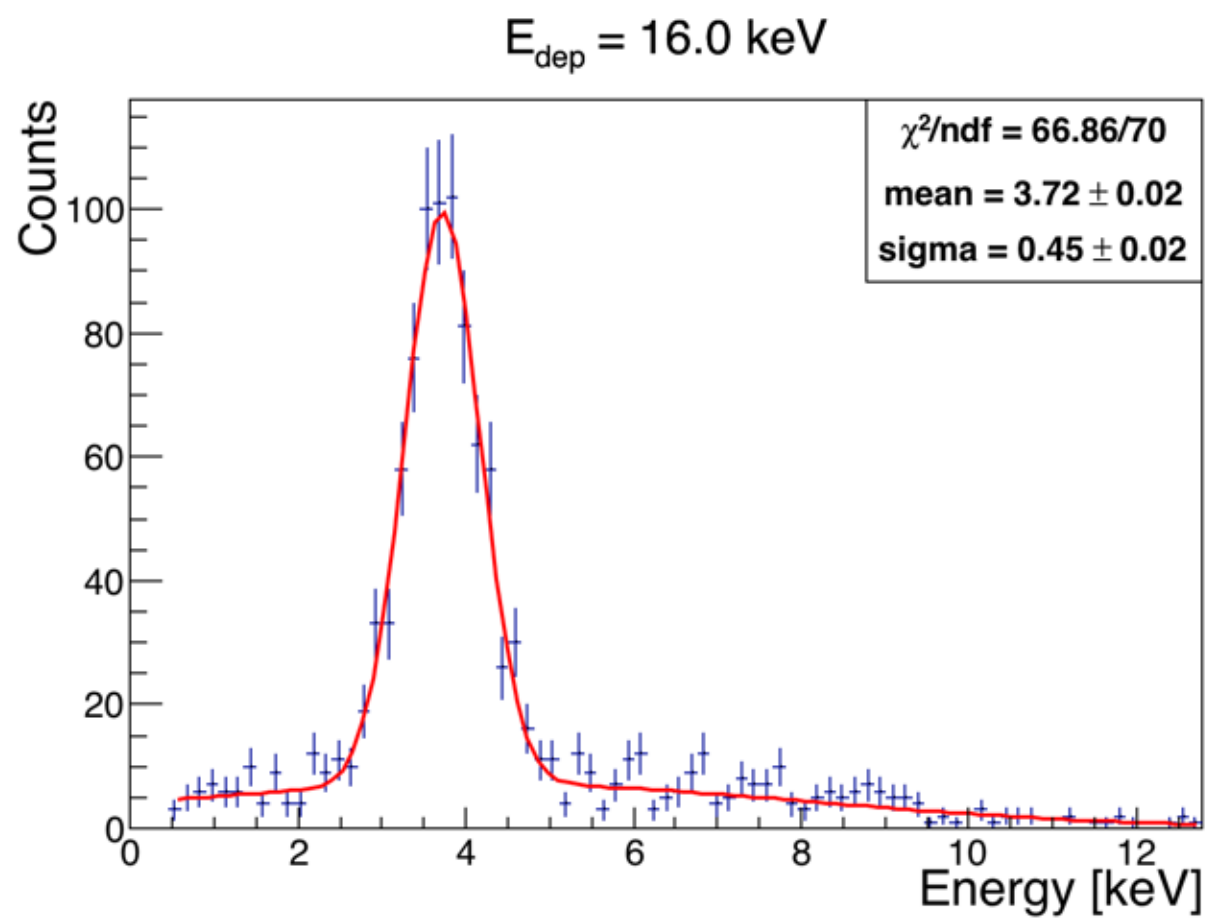

Figure 4.32: Raw data and fit for $16.0 \mathrm{keV}$ nuclear recoil depositions.

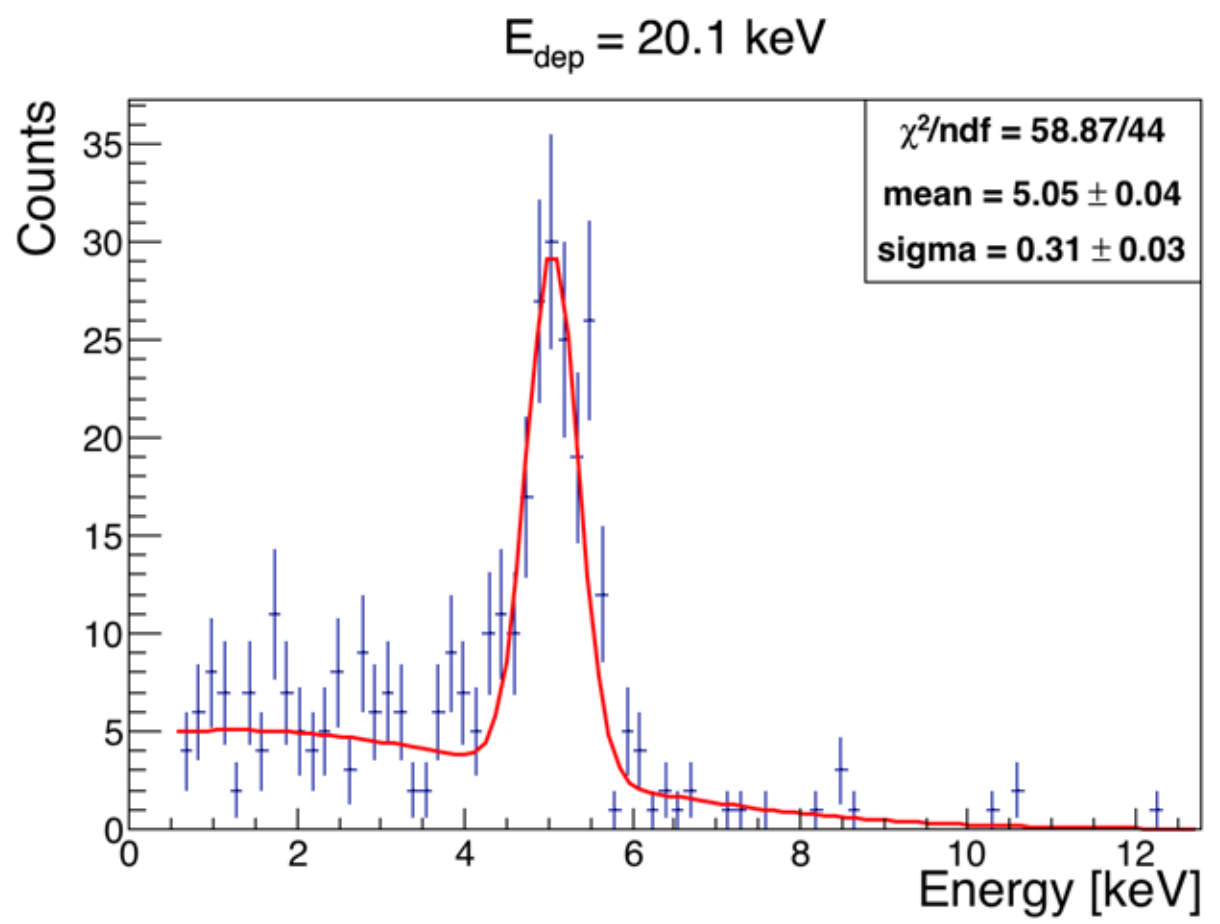

Figure 4.33: Raw data and fit for $20.1 \mathrm{keV}$ nuclear recoil depositions. 


\section{$E_{\text {dep }}=25.3 \mathrm{keV}$}

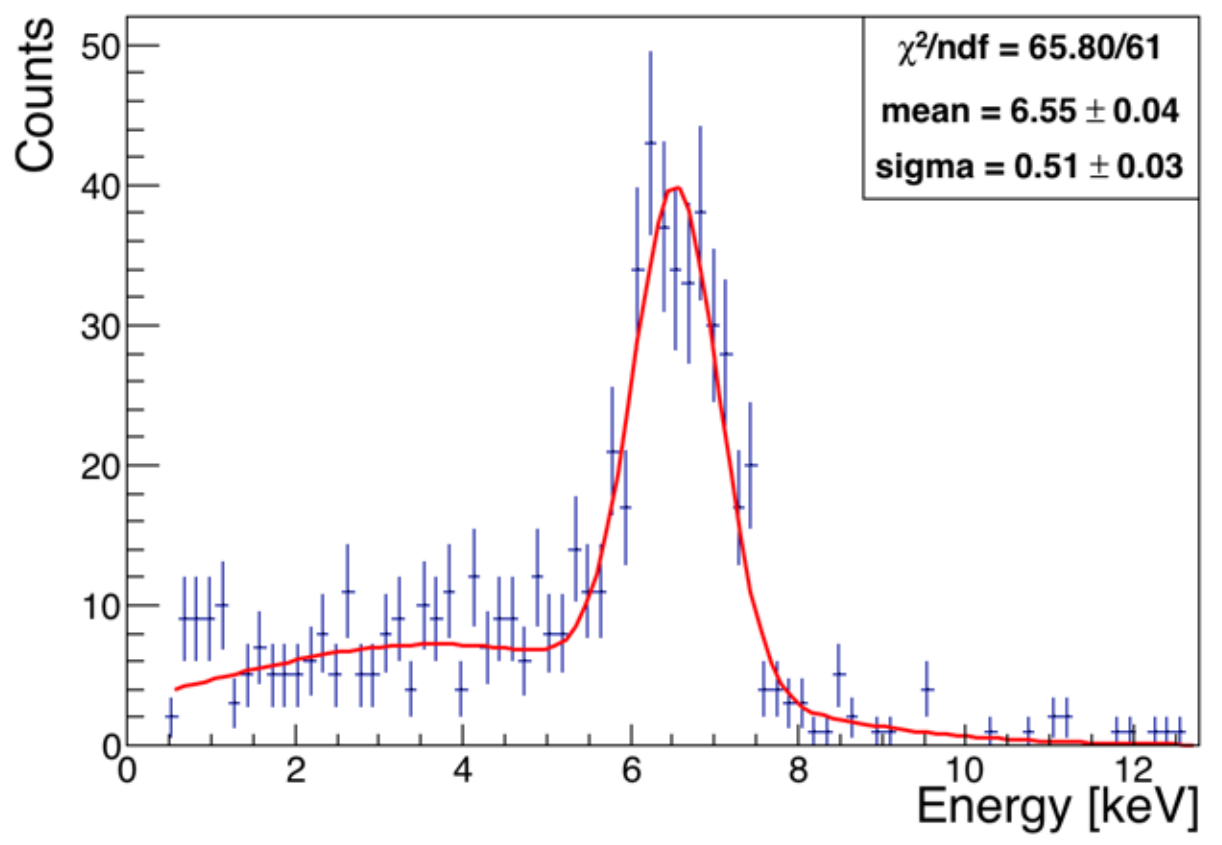

Figure 4.34: Raw data and fit for $25.3 \mathrm{keV}$ nuclear recoil depositions.

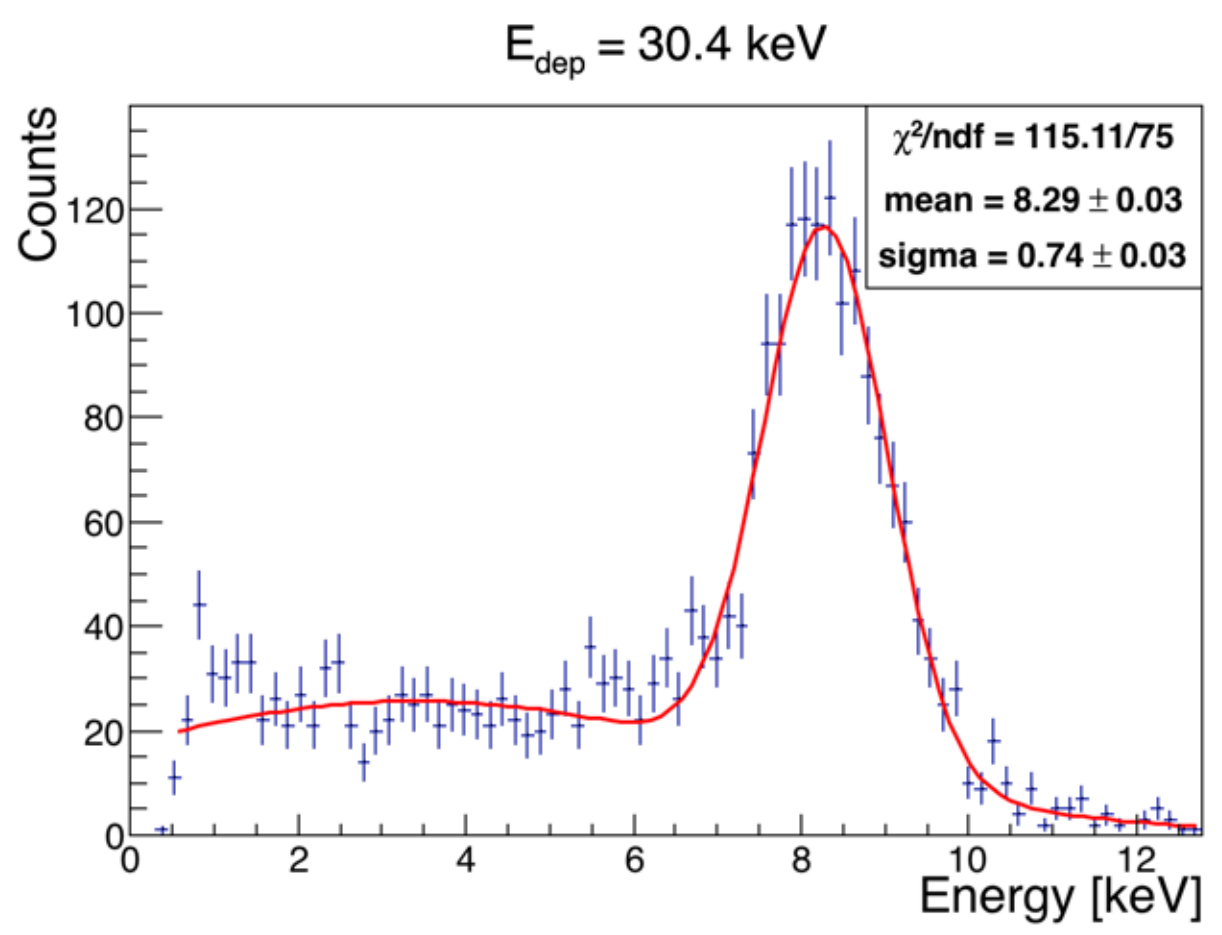

Figure 4.35: Raw data and fit for $30.4 \mathrm{keV}$ nuclear recoil depositions. 


\section{SUMMARY}

\subsection{Detector Fabrication}

A robust, repeatable fabrication procedure has been established, demonstrated, and improved at the TAMU fabrication facility. Increased throughput as well as improvements made in the process itself are expected to contribute substantially to the success of the next generation SuperCDMS SNOLAB experiment. In particular, increased fabrication efficiency, improved TES $\mathrm{T}_{c}$ consistency and uniformity, increased bias voltage ability due to substrate trenching, and improved signal collection from overhang studies will improve detector success rates, reducing fabrication and testing costs.

\subsection{Low Energy Nuclear Recoil Measurements}

This work has provided a confident measurement of ionization yields of nuclear recoils in the relevant energy range of today's direct detection experiments. A platform and technique has been established allowing future measurements to be made at lower energies, as these will be needed for the next generation of dark matter search experiments. Using the Lindhard equation defined by Lindhard et. al [29], a best-fit k-value of 0.146 was found (see Figures 4.24 and 4.25).

Implications for future work have been mentioned throughout this work, including:

- Measurements using voltage assisted calorimetric ionization detectors (see Section 2.4.2) to probe lower energies using the method outlined here

- Prioritization of scatter parameter choices (not only lower energy measurements, but improved resolution). See section 4.2.6. 
- Implementation of neutron:gamma discrimination in scatter detectors

- Rigorous fitting model for data analysis 


\section{REFERENCES}

[1] F. Zwicky. The redshift of extragalactic nebulae. Helv. Phys. Acta, 6:110, 1933.

[2] P.A.R. Ade, N. Aghanim, M.I.R. Alves, C. Armitage-Caplan, M. Arnaud, M. Ashdown, F. Atrio-Barandela, J. Aumont, H. Aussel, C. Baccigalupi, et al. Planck 2013 results. I. overview of products and scientific results. Astronomy $\&$ Astrophysics, 571:A1, 2014.

[3] J. Einasto, A. Kaasik, and E. Saar. Dynamic evidence on massive coronas of galaxies. Nature, 250:309-310, 1974.

[4] D. Clowe, M. Bradač, A.H. Gonzalez, M. Markevitch, S.W. Randall, C. Jones, and D. Zaritsky. A direct empirical proof of the existence of dark matter. The Astrophysical Journal Letters, 648(2):L109, 2006.

[5] J.D. Lewin and P.F. Smith. Review of mathematics, numerical factors, and corrections for dark matter experiments based on elastic nuclear recoil. Astroparticle Physics, 6(1):87-112, 1996.

[6] D. Moore. A search for low-mass dark matter with the cryogenic dark matter search and the development of highly multiplexed phonon-mediated particle detectors. California Institute of Technology Dissertation, 2012.

[7] N. Mirabolfathi, H.R. Harris, R. Mahapatra, K. Sundqvist, A. Jastram, B. Serfass, D. Faiez, and B. Sadoulet. Toward single electron resolution phonon mediated ionization detectors. arXiv:1510.00999. 2015.

[8] J. Sander, Z. Ahmed, A.J. Anderson, S. Arrenberg, D. Balakishiyeva, R.B. Thakur, D.A. Bauer, D. Brandt, P.L. Brink, R. Bunker, et al. SuperCDMS status from Soudan and plans for SNOLab. In Workshop on dark matter, unifi- 
cation and neutrino physics: CETUP* 2012, volume 1534, pages 129-135. AIP Publishing, 2013.

[9] B.E. Warren. X-ray Diffraction. Dover Publications, Mineola, NY, USA, 1990.

[10] P.J. Holmes and P. Handler. The electrochemistry of semiconductors. Journal of The Electrochemical Society, 109(8):228C, 1962.

[11] P.L. Brink, Z. Ahmed, D.S. Akerib, C.N. Bailey, D. Balakishiyeva, D.A. Bauer, J. Beaty, R. Bunker, B. Cabrera, D.O. Caldwell, et al. SuperCDMS detector fabrication advances. In The Thirteenth International Workshop on Low Temperature Detectors-LTD13, volume 1185, pages 655-658. AIP Publishing, 2009.

[12] P.L. Brink, A.J. Anderson, D. Balakishiyeva, D.A. Bauer, J. Beaty, D. Brandt, B. Cabrera, H. Chagani, M. Cherry, J. Cooley, et al. Detector fabrication yield for SuperCDMS Soudan. Journal of Low Temperature Physics, 176:1-7, 2014.

[13] K. Prasad. Search for lightly ionizing particles using CDMS-II data and fabrication of CDMS detectors with improved homogeneity in properties. Texas A\&M University Dissertation, College Station, TX, 2013.

[14] K.R. Williams and R.S. Muller. Etch rates for micromachining processing. Journal of Microelectromechanical Systems, 5(4):256-269, 1996.

[15] C. James. The residual resistance of metals. Physics Education, 2(6):315, 1967.

[16] E. Lassner and W.D. Schubert. Tungsten: properties, chemistry, technology of the elements, alloys, and chemical compounds. Springer, 1999.

[17] A.E. Lita, D. Rosenberg, S. Nam, A.J. Miller, D. Balzar, L.M. Kaatz, and R.E. Schwall. Tuning of tungsten thin film superconducting transition temperature for fabrication of photon number resolving detectors. Applied Superconductivity, IEEE Transactions on, 15(2):3528-3531, 2005. 
[18] B.A. Young, T. Saab, B. Cabrera, J.J. Cross, R.M. Clarke, and R.A. Abusaidi. Measurement of $\mathrm{T}_{c}$ suppression in tungsten using magnetic impurities. Journal of Applied Physics, 86(12):6975-6978, 1999.

[19] N.E. Booth. Quasiparticle trapping and the quasiparticle multiplier. Applied physics letters, 50(5):293-295, 1987.

[20] R. Agnese, A.J. Anderson, D. Balakishiyeva, R.B. Thakur, D.A. Bauer, A. Borgland, D. Brandt, P.L. Brink, R. Bunker, B. Cabrera, et al. Demonstration of surface electron rejection with interleaved germanium detectors for dark matter searches. Applied Physics Letters, 103(16):164105, 2013.

[21] S. Hertel. Advancing the search for dark matter: from CDMS II to SuperCDMS. Massachusetts Institute of Technology Dissertation, Cambridge, MA, 2012.

[22] M. Pyle. Optimizing the design and analysis of cryogenic semiconductor dark matter detectors for sensitivity. Stanford University Dissertation, Stanford, CA, 2012.

[23] P.L. Brink, B. Cabrera, J.P. Castle, J. Cooley, L. Novak, R.W. Ogburn, M. Pyle, J. Ruderman, A. Tomada, B.A. Young, J. Filippini, P. Meunier, N. Mirabolfathi, B. Sadoulet, D.N. Seitz, B. Serfass, K.M. Sundqvist, D.S. Akerib, C.N. Bailey, M.R. Dragowsky, D.R. Grant, R. Hennings-Yeomans, and R.W. Schnee. First test runs of a dark-matter detector with interleaved ionization electrodes and phonon sensors for surface-event rejection. Nuclear Instruments and Methods in Physics Research Section A: Accelerators, Spectrometers, Detectors and Associated Equipment, 559(2):414 - 416, 2006.

[24] M. Pyle, B. Serfass, P.L. Brink, B. Cabrera, M. Cherry, N. Mirabolfathi, L. Novak, B. Sadoulet, D. Seitz, K.M. Sundqvist, et al. Surface electron rejection from Ge detector with interleaved charge and phonon channels. In The Thirteenth 
International Workshop on Low Temperature Detectors-LTD13, volume 1185, pages 223-226. AIP Publishing, 2009.

[25] D.S. Akerib, J. Alvaro-Dean, M.S. Armel-Funkhouser, M.J. Attisha, L. Baudis, D.A. Bauer, J. Beaty, P.L. Brink, R. Bunker, S.P. Burke, et al. First results from the Cryogenic Dark Matter search in the Soudan Underground Laboratory. Physical Review Letters, 93(21):211301, 2004.

[26] B. Shank, D.Q. Nagasawa, B. Cabrera, M. Cherry, and B.A. Young. Charge transport asymmetry in cryogenic high purity germanium. Journal of Low Temperature Physics, pages 1-7, 2014.

[27] DOW Chemical Company. Shipley Microposit S1800 Series Photo Resists Data Sheet, (accessed may 2014), http://www.microchem.com/pdfs'dow/s1800.pdf.

[28] R. Agnese, Z. Ahmed, A.J. Anderson, S. Arrenberg, D. Balakishiyeva, R.B. Thakur, D.A. Bauer, J. Billard, A. Borgland, D. Brandt, et al. Silicon detector dark matter results from the final exposure of CDMS II. Physical review letters, 111(25):251301, 2013.

[29] J. Lindhard and M. Scharff. Energy dissipation by ions in the keV region. Physical Review, 124(1):128, 1961.

[30] J. Lindhard, V. Nielson, M. Scharff, and P.V. Thomsen. Integral equations governing radiation effects. Dan. Vidensk. Selsk. Mat. Fys. Medd, 33(10), 1963.

[31] J. Lindhard, M. Scharff, and H.E. Shiott. Range concepts and heavy ion ranges. Dan. Vidensk. Selsk. Mat. Fys. Medd, 33(14), 1963.

[32] S.T. Lin, H.B. Li, X. Li, S.K. Lin, H.T. Wong, M. Deniz, B.B. Fang, D. He, J. Li, C.W. Lin, et al. New limits on spin-independent and spin-dependent couplings 
of low-mass WIMP dark matter with a germanium detector at a threshold of $220 \mathrm{eV}$. arXiv:0712.1645v4.

[33] K.H. Beckurts and K. Wirtz. Neutron Physics. Springer-Verlag, Berlin, Germany, 1964.

[34] C.L. Lee and X.L. Zhou. Thick target neutron yields for the ${ }^{7} \mathrm{Li}(\mathrm{p}, \mathrm{n}){ }^{7} \mathrm{Be}$ reaction near threshold. Nuclear Instruments and Methods in Physics Research Section B: Beam Interactions with Materials and Atoms, 152(1):1-11, 1999.

[35] National Institute of Standards and Technology. PSTAR Database, (accessed October 2015), http://physics.nist.gov/physrefdata/star/text/pstar.html.

[36] B. Wolf. Handbook of Ion Sources. CRC press, NY, USA, 1995.

[37] National Electrostatics Corporation. Peletron Charging System, (accessed April 2015), http://www.pelletron.com/charging.htm. 


\title{
APPENDIX A
}

\section{DUOPLASMATRON ION SOURCE}

\begin{abstract}
A.1 Overview
The ion source used in this work is a helium-fed duoplasmatron, named as such because it is a plasmatron source with two plasma regions [36]. It is used to produce a $22 \mathrm{keV} \mathrm{H}^{-}$beam to inject into the a tandem accelerator, which converts the beam into protons during acceleration. A tungsten filament is used to emit electrons allowing an arc to strike and be maintained in a rarefied ( 100mTorr) $\mathrm{H}_{2}$ atmosphere (see Figure A.1). In the plasma subsequently formed, some of the hydrogen is ionized into $\mathrm{H}^{-}$and accelerated through the anode aperture (0.040" in this case). Within the Zwischen (the chamber/electrode in which the filament is located), the charged particles are initially focused with an electromagnet. Once through the anode aperture, the beam encounters an electrostatic focusing assembly. This assembly has two permanent magnets mounted at the entrance (in same directional polarity) to sweep away the lighter electrons that would otherwise pollute the beam. This takes place within a 22 $\mathrm{kV}$ pre-acceleration column, which accelerates the beam for injection into the main accelerator.
\end{abstract}

\section{A.2 Anode}

The anode aperture has been carefully chosen to be large enough to allow a high beam current, but not so high that the hydrogen gas-load overpowers downstream pumps. In this case the aperture, initially 0.025 " from the manufacturer, was machined out to 0.040 " to meet these requirements. It should be noted that high electromagnet currents, while improving beam current, cause the initial beam 


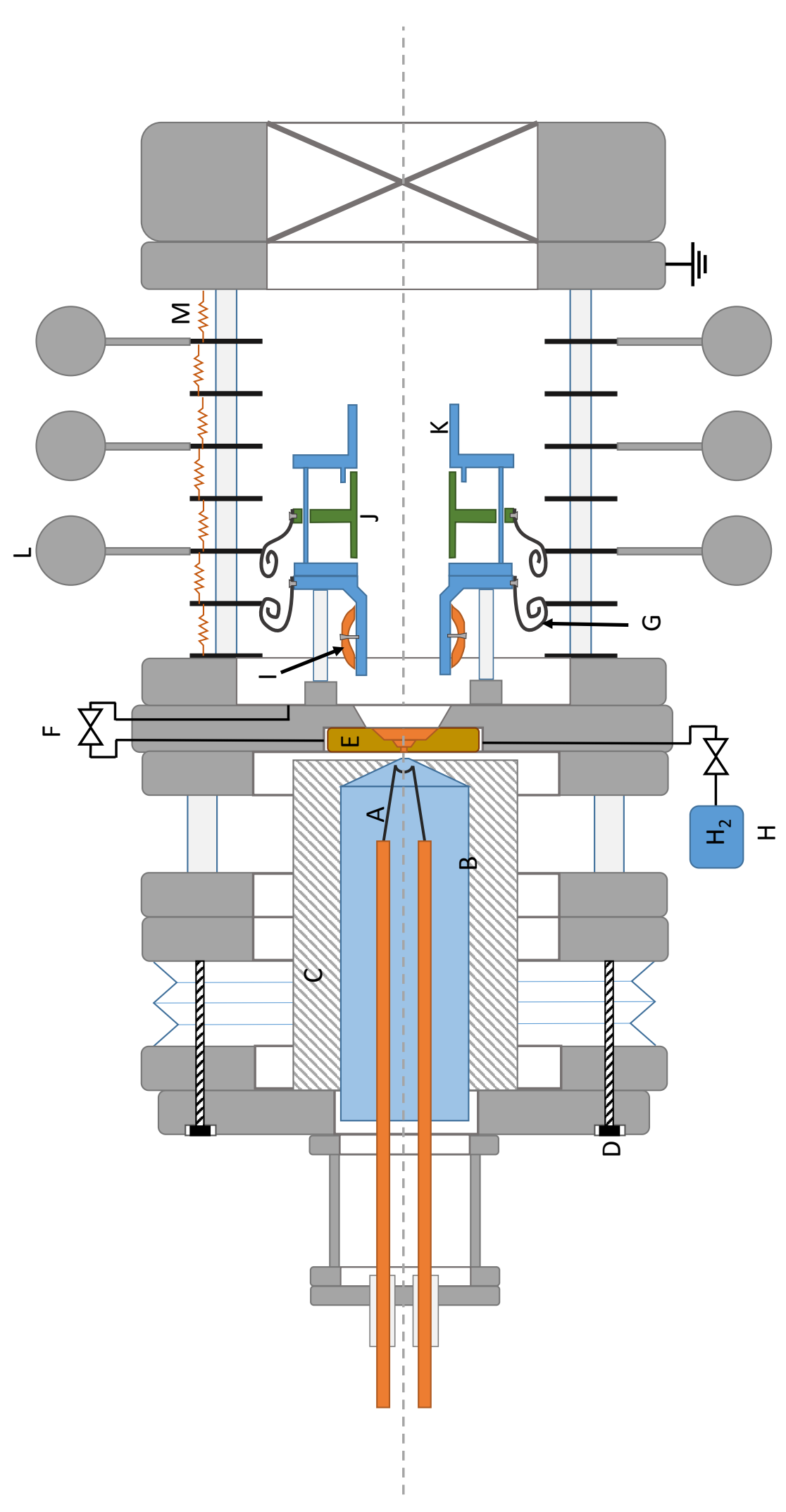

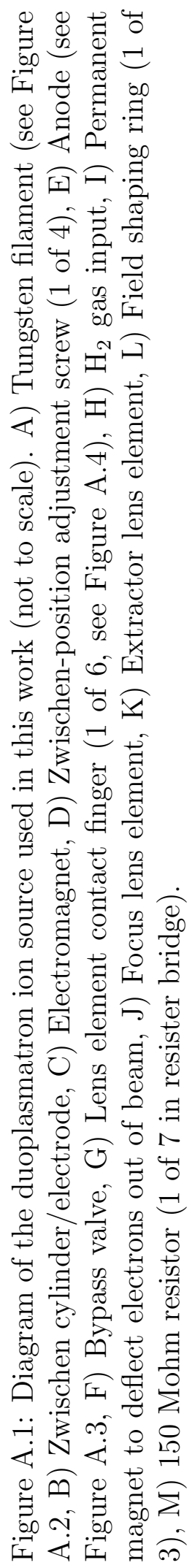




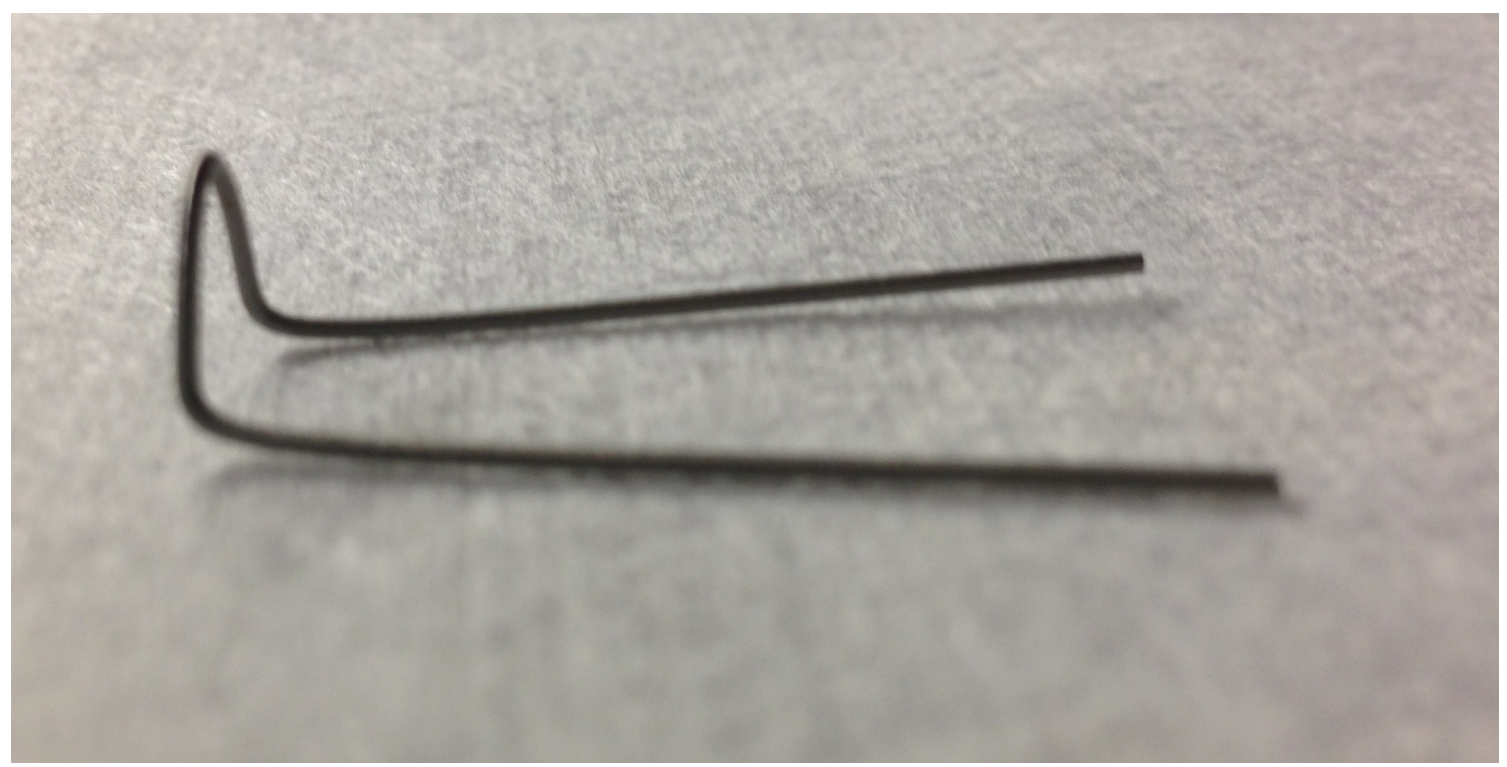

Figure A.2: Example filament used in this ion source. It is 0.027 " diameter tungsten wire, cut to $2.75 "$, then manually bent using needle-nose pliers.

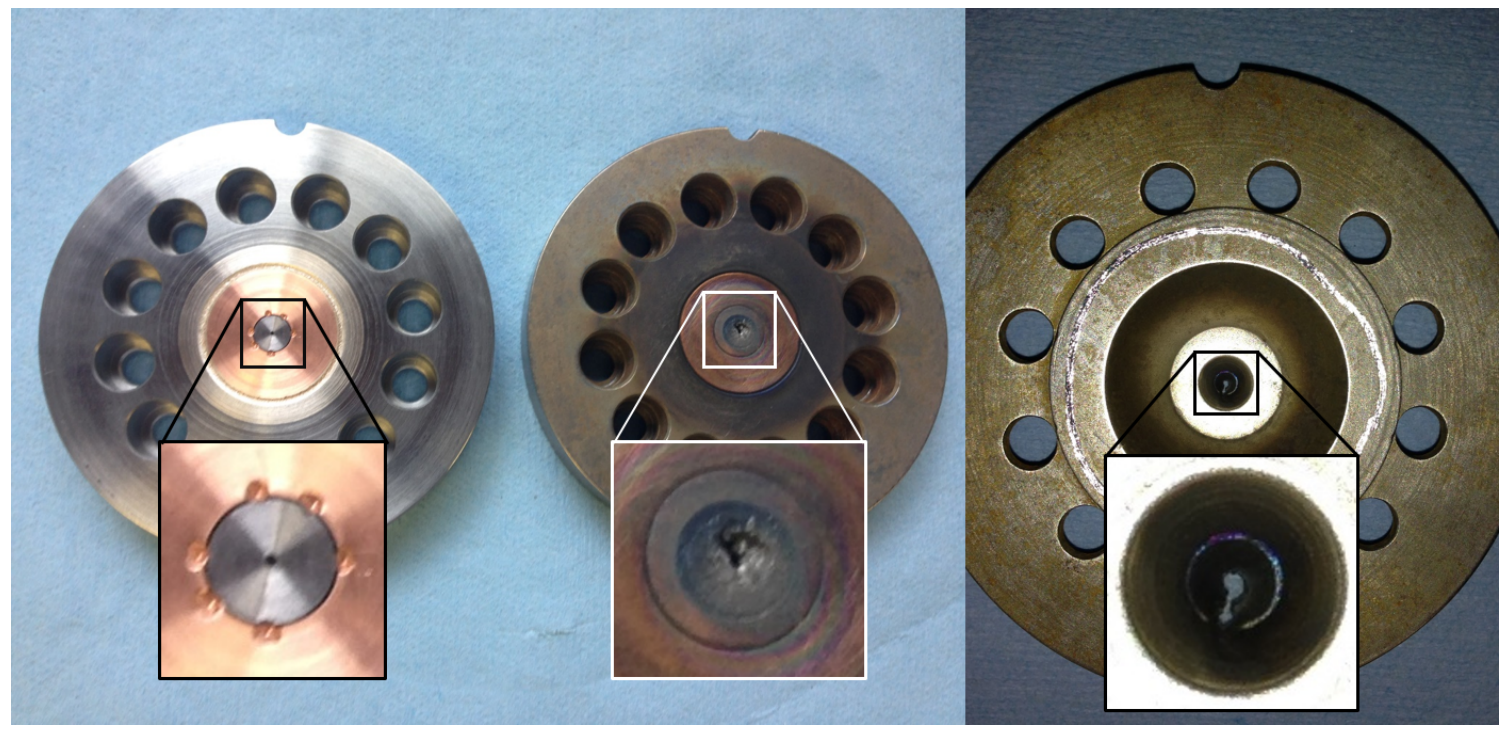

Figure A.3: Left) Comparison of damaged anode next to its replacement. Note the damage near the aperture from extended abuse from the beam staying in a single spot. Right) The rear (beam exiting) side of the damaged anode, showing that the damage from the front, not only punched through, but caused an irregular shape in the aperture. This extra hole resulted in extra errant beam, which subsequently loaded down the lens elements (their power supplies), preventing successful beam operation. 


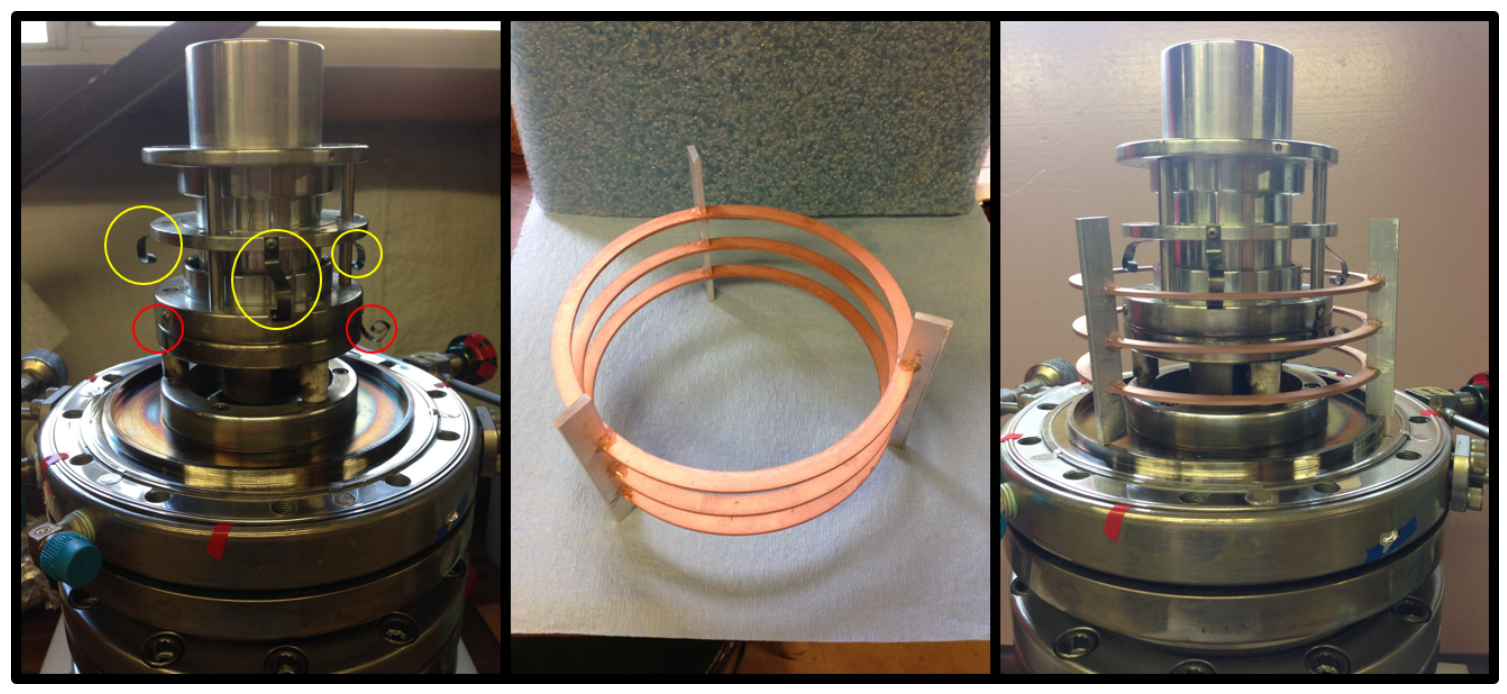

Figure A.4: Ion source lens elements and accelerator column simulation jig. Left) Yellow circles indicate focus electrode "fingers", whereas red circles indicate the equivalent component locations on the extractor electrode (missing "finger" on left has since been replaced. Center) Jig created to simulate the inner ring contact points of the accelerator column, allowing lens element "fingers" to be shaped appropriately before blind assembly. Right) Jig mounted on ion source to test the configuration of contact "fingers".

to drill into the anode quite violently (see Figure A.3). To extract the appropriate H ions $\left(\mathrm{H}^{-}\right.$versus $\left.\mathrm{H}^{+}\right)$, the Zwischen aperture must be offset from the anode aperture. This offset can be positioned in different directions around the anode to spread out this damage and prolong the anode lifetime.

\section{A.3 Lens Elements}

The lens elements within the ion source are bolted to the anode flange, but receive their voltage/power from external electrical connections on the resistor bridge (see Figure A.1). Upon assembly, the lens elements make contact with those exterior nodes via flexible metal fingers (see Figure A.4). These fingers must be properly bent such that they provide firm enough contact but can't protrude so much that they catch on the other accelerator column contacts upon insertion. Unfortunately, 
these fingers are out of view during assembly, so they must be blindly shaped before re-assembly. To ensure proper shape was formed, a custom jig was created to simulate the interior of the accelerator column. Using this jig, the fingers can be bent and tested while allowing visual inspection and allowing the user to physically feel and adjust the contact pressure. This was required during a rebuild of the ion-source during this work. 


\section{APPENDIX B}

\section{PELLETRON TANDEM ACCELERATOR}

The accelerator used in this work is a Pelletron 2UDH tandem accelerator built by National Electrostatics Corporation (NEC), and initially commisioned in 1973. It was designed to operate at a nominal $2 \mathrm{MV}$, making it suitable for accelerating protons up to $4 \mathrm{MeV}$. This is accomplished by injecting a pre-accelerated beam of $\mathrm{H}^{-}$into the accelerator tank, where it accelerates toward the $\leq 2 \mathrm{MV}$ terminal potential. At this point (in the center of the tank), the beam encounters a very thin $\left(\sim 10 \mu \mathrm{g} / \mathrm{cm}^{2}\right)$ carbon foil. The carbon strips the electrons from the $\mathrm{H}^{-}$particles, leaving a beam of protons, changing the charge polarity and causing them to accelerate back to ground potential as they exit the other end.

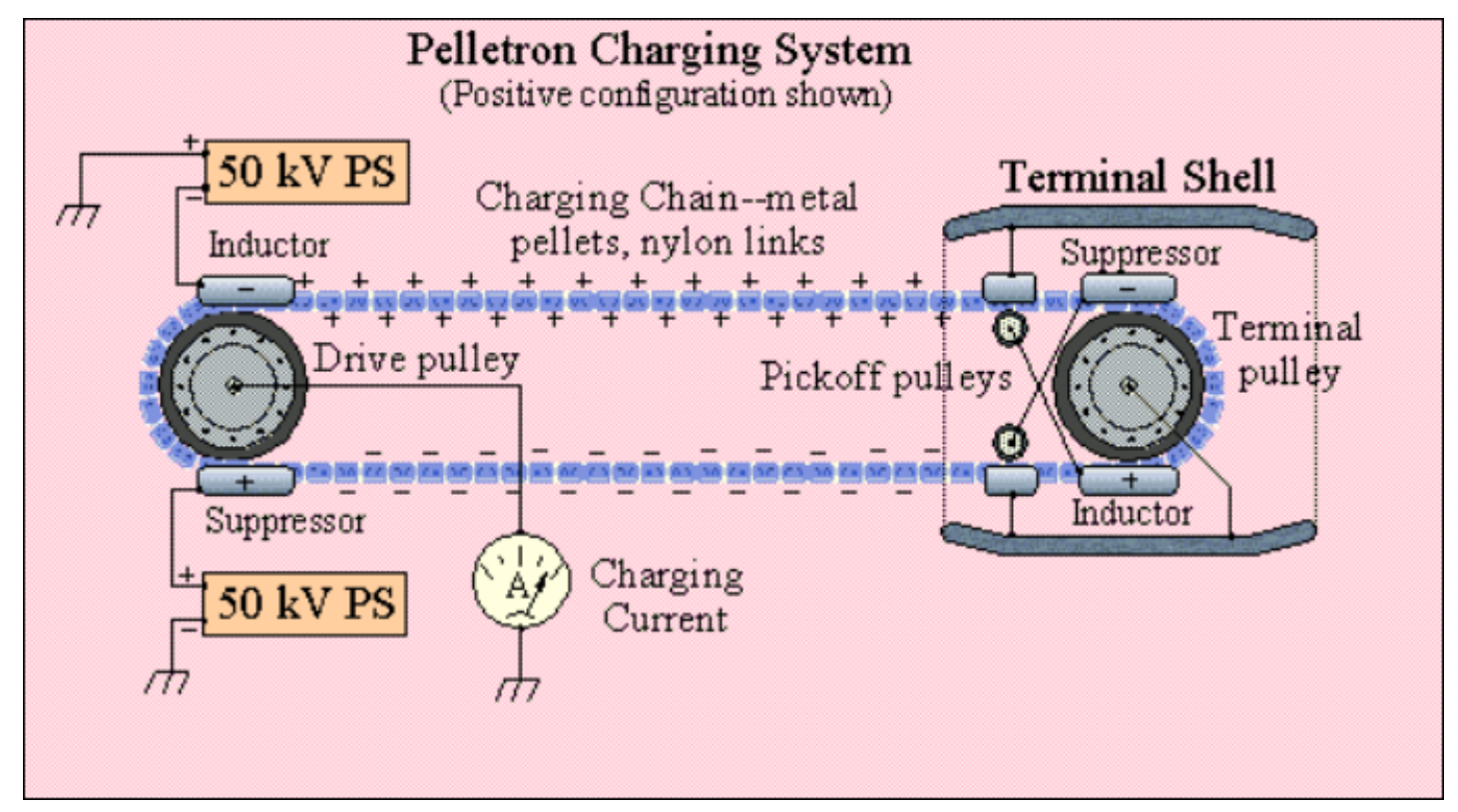

Figure B.1: Diagram of pelletron charging system [37]. Chain and pulleys rotate clockwise (see reference for online animation). 
The terminal potential is maintained by the Pelletron charging system (see Figure B.1). This system uses a chain consisting of insulating links connecting metal cylinders (aka pellets). Charge is driven on and off of these links by inductors located at the ground and high voltage terminal pulleys. In this way, it acts as an electron conveyor belt, robbing the terminal potential of electrons, creating a node of high voltage. Nominally this system should be able to provide a 3:1 ( $\mu \mathrm{A}$ charging current:kV charging voltage) ratio. Throughout this work, a ratio of 1.5-2:1 was typically achieved, sufficient for the voltages used $(\leq 1.9 \mathrm{MV})$. The chain stretches with use, requiring links to be removed routinely (approximately annually depending on use). The main symptom indicating this maintenance is required is the inability of the charging system to maintain potential for long periods of time (hours of continual use). Two links were removed in the process of this experiment.

The ability of the charging system to maintain high voltage is supported by the pressurized $\mathrm{SF}_{6}$ atmosphere in which it resides. The system nominally operates with 80psig of this gas, however, for the voltages used in this experiment, a range from 60-77psig was used with success. This value is heavily driven by the cost of the gas.

To perform maintainence inside the $\mathrm{SF}_{6}$ tank (the accelerator tank), the gas first must be evacuated and transferred to the dedicated storage tank (maintained otherwise at $1 \mathrm{~atm} \mathrm{SF}_{6}$ ). This is done by a custom gas transfer system plumbed to the two. First, the accelerator tank pressure is routed directly to the storage tank to equalize the two. Second, a compressor is used in between to assist in pumping to the storage tank. This is done until the accelerator tank reaches 1atm. At this point a vacuum pump is used to extract the $\mathrm{SF}_{6}$ from the accelerator tank, sending the gas into the compressor which then pumps it into the storage tank. In this way, the expensive gas can be salvaged for re-use. The accelerator tank can then be vented to atmosphere and opened. To refill the accelerator tank after closing, it is first connect 
to a vacuum pump which pumps its contents out to atmosphere. When sufficient vacuum is achieved, the tanks are then equalized, then the compressor is used to transfer the $\mathrm{SF}_{6}$ back until the storage tank is back down to 1atm. The storage tank is stored at $1 \mathrm{~atm}$ in case there are leaks (the $\mathrm{SF}_{6}$ will not escape and atmosphere gas will not enter). Often after an $\mathrm{SF}_{6}$ transfer, additional $\mathrm{SF}_{6}$ is needed to make up for losses in the process. This gas is routed into the storage tank while the compressor pumps the storage contents into the accelerator tank.

Another issue that can arise after gas transfer is that moisture can become incorporated in the $\mathrm{SF}_{6}$. For this reason, there is a separate dryer circuit used to dry the gas. It is composed of a filter, a blower, and a desiccant bed. After a number of drying cycles, this desiccant must be revived. This is accomplished by running compressed air through the bed while heating the desiccant with the built in heater. This air is then vented to atmosphere carrying a good deal of moisture with it. This process was performed once during this experiment.

While the $\mathrm{SF}_{6}$ is used to prevent arcing and charge loss, some amount of charge loss (when carefully controlled) is actually desirable. This comes in the form of corona discharge current from the high voltage terminal to the 'corona point' mounted on the outer tank. This needle is a regular sewing needle purchased locally used as a lightning within the tank. It can be driven closer to or further from the terminal to control the current extracted (typically $\sim 30 \mu \mathrm{A}$ ).

With the main control of terminal potential being the charging voltage, the critical, real-time, fine-tuning corrections are made be another dedicated system, called the TPS (terminal potential stabilizer). The system uses two current-collecting slits at the end of the accelerated, bent beam-line to monitor whether the beam is being bent to much or two little by the bending magnet. Since the magnetic field has been set according to our desired beam energy, these errors are not seen as the beam being 
'over- or under-bent', but rather too energetic or not energetic enough. While the most likely fluctuation would be an un-regulated terminal potential, as it needs to be held constant to $\sim 0.1 \%$, there are many focusing and steering elements which could have mild fluctuations. Regardless, these are compensated for by the TPS in real-time by making constant changes to the charging/discharging currents. 